\title{
HAMBURG RADIOCARBON THIN LAYER SOILS DATABASE
}

\section{PETER BECKER-HEIDMANN, HANS-WILHELM SCHARPENSEEL and HORST WIECHMANN}

Institut für Bodenkunde, Universität Hamburg, Allende-Platz 2, 20146 Hamburg, Germany

ABSTRACT. We report here the remainder of the Hamburg University dates on thin soil layers (HAM 1652-3129).

\section{INTRODUCTION}

Soils and especially soil organic matter (SOM) have been objects of radiocarbon dating since the early days of the technique (Scharpenseel and Becker-Heidmann 1992). The first major objective, to determine the "age" or starting point of the development of a specific soil, is still open to methodological questions. On the other hand, natural ${ }^{14} \mathrm{C}$ measurements have been successful in providing information on the dynamics of SOM. Chemical and physical fractions of SOM have been "dated" separately to study the mechanisms of turnover, humification and stabilization. Interest in pedogenesis and the processes of soil development motivated the dating of several or all horizons of a soil profile. In the 1970s, with the increase in global environmental research and computer modeling of the major nutrient cycles, demand grew for more detailed information on soil carbon inventory and fluxes. SOM represents the second largest organic pool in the global carbon cycle, comprising 1200 to $1500 \mathrm{Pg}$ of $\mathrm{C}$ in the $12.8 \times 10^{9}$ ha of ice-free terrestrial soils and $300-500 \mathrm{Pg}$ of C in the $6.3 \times 10^{8}$ ha of wetlands (Scharpenseel and Becker-Heidmann 1994b).

In 1981 we started sampling and dating entire soil profiles as thin layers. Trying to cover the important soil classes, we have now completed ${ }^{14} \mathrm{C}$ and $\delta^{13} \mathrm{C}$ measurements on 26 profiles and set up a database in dBASE IV. Here, we present these data together with available additional information according to the specifications of the planned International Radiocarbon Soils Data Base (IRSDB) (Becker-Heidmann 1996). Our intention is to provide the modeling community with test and reference soil data. Useful examples of applications were first published by Harrison et al. (1993) and Becker-Heidmann et al. (1995).

\section{METHODS}

The soil samples were taken as successive complete $2-\mathrm{cm}$ thin layers from the top to the bottom of the profile, using trowel, meter and water level to control depth and horizontal plane, as described by Becker-Heidmann and Scharpenseel (1986). After air drying and transport to the laboratory in Hamburg, roots and stones were removed. Each sample was carefully homogenized, followed by sieving to $2 \mathrm{~mm}$ and drying at $105^{\circ} \mathrm{C}$, before subsamples were taken for the different analyses. We measured $\mathrm{pH}$ in a $0.01 \mathrm{M} \mathrm{CaCl}_{2}$ solution, and tested for the presence of $\mathrm{CaCO}_{3}$.

Organic and inorganic carbon content were measured conductometrically using a Wösthoff apparatus and were separated by temperature (Becker-Heidmann 1990). For the determination of $\delta^{13} \mathrm{C}$, the samples were combusted in an oxygen stream. The $\mathrm{CO}_{2}$ was purified in a vacuum line and trapped. Details on the preparation line with computer-controlled cooling traps are given in BeckerHeidmann (1989). The measurements were carried out using a high-precision isotope mass spectrometer (Finnigan MAT 250). The natural ${ }^{14} \mathrm{C}$ concentrations were determined as described by Becker-Heidmann $(1989,1990)$, via combustion and conversion of the sample carbon to strontium carbonate, benzene synthesis and measurement by liquid scintillation spectrometry. Some samples with very low carbon content were augmented with $\mathrm{CO}_{2}$ free of ${ }^{14} \mathrm{C}$ (Scharpenseel and Pietig 1970). 


\section{SOIL DATABASE}

At present, the database contains the measurement results of $\mathrm{pH}$, carbon content, $\delta^{13} \mathrm{C}$ and ${ }^{14} \mathrm{C}$ activity of 26 complete profiles. Additionally, the conventional ${ }^{14} \mathrm{C}$ age (Stuiver and Polach 1977) is given here for organic matter of $\mathrm{C}_{3}$ plant origin, i.e., corrected for isotope discrimination to a nominal $\delta^{13} \mathrm{C}$ value of $-25 \%$ PDB. Rice (Oryza sativa $\mathrm{L}$.), for example, is a $\mathrm{C}_{3}$ plant. For carbonates and $\mathrm{C}_{4}$-plant-derived organic matter (e.g., sorghum and tropical grasses) the ${ }^{14} \mathrm{C}$ age is calculated without isotope correction.

We also list here all available information on the soil profiles relevant for further use in modeling SOM dynamics. The profile description is kept brief; where the detailed results have already been published, reference is made to the corresponding literature.

The profiles are grouped according to their hydrological, climatic and management regime and their location.
A Terrestrial soils
A1 Humid climate
A1a Forest soils
Ohlendorf Forest, Profile A
Ohlendorf Forest, Profile B
Wohldorf Forest
Timmendorf Forest
Trittau Forest
A1b Agricultural soils
Klein Altendorf
Savarit, Profile 1
Savarit, Profile 2
A2 Mediterranean Climate
Akko
Qedma
A3 Semiarid Climate
Patancheru, Profile $P$
Patancheru, Profile R
B Wetland soils
Los Baños
Pangil
Pao
Bugallon
Tiaong, Profile L
Tiaong, Profile $\mathrm{H}$
San Dionisio, Profile T
Namtou Hsien
Pingtung
Chum Pae
Klong Luang
Tachiat, Profile 1
Tachiat, Profile 2
Tonsang 


\section{ACKNOWLEDGMENTS}

These studies started in 1981 and have been financially supported mainly by the Deutsche Forschungsgemeinschaft (DFG, Contract Scha 47/23), the German Federal Ministry of Economic Cooperation (BMZ) through the Gesellschaft für Technische Zusammenarbeit (GTZ, Contract no. 72.7866.6-01.400/1420) and the European Union (EU, subcontract with ESA). We appreciate the cooperation of the École Supérieure d'Agriculture d'Angers (ESA), France; the Hebrew University of Jerusalem, Israel; the International Crops Research Institute for the Semi-Arid Tropics (ICRISAT), India; the International Rice Research Institute (IRRI), Philippines; the Asian-Pacific Food and Fertilizer Technology Center (ASPAC-FFTC), Taiwan; the Department of Agriculture (DOA), Thailand; and the Prince of Songkhla University, Thailand. We wish to thank the numerous colleagues who assisted in the completion of this work, in either profile selection, sampling, sample preparation or discussion of the results, namely O. Andresen, T. Arayangkoon, A. Aveline, S. Bielfeldt, G. Boje-Klein, N. Bonkeerd, I. Briese, J. Burford, R. Busch, J. M. Chang, P. Chawainakupt, Y. Crozat, A. M. Domenach, I. Drachenberg, M. Eberle, E. Eichwald, H. Y. Guo, D. Heidmann, B. Hintze, W. M. Hsiang, Y. M. Huang, M. Juanico, T. C. Juang, A. Jordan, D. Kalmar, A. Kamnalrut, S. Koteponge, B. Klimaschka, R. S. Lantin, J. Ludwig, B. Lüssem, S. Manit, H. Mantler, U. Martin, H. U. Neue, M. R. Orticio, S. Osterholz, M. Pallul, Pandu, S. Phongpan, K. Poremba, C. C. Quijano, T. J. Rego, Rudi, Greg and Rocco of IRRI, K. L. Sahrawat, A. Satrusajang, W. C. Schulz, J. Schuster, N. Seidel, V. P. Singh, P. Snitwongse, S. Suthipradit, Syedali, V. Toquillo, S. Virmani, M. Vollmer, 24 women fieldworkers of ICRISAT, Dr. Wong, M. Wurzer, and D. H. Yaalon. Last but not least the first author is very grateful to his wife and children, to his co-authors, to A. Long, R. Kra and D. D. Harkness for their great patience and to D. Sewell for his thorough work on the manuscript.

\section{REFERENCES}

Andresen, O. (ms.) 1987 Untersuchung der Isotopenverhältnisse an kalkhaltigen tiefgründigen Vertisolen aus Israel. Diploma Thesis, University of Hamburg.

Becker-Heidmann, P. 1989 Die Tiefenfunktionen der natürlichen Kohlenstoff-Isotopengehalte von vollständig dünnschichtweise beprobten Parabraunerden und ihre Relation zur Dynamik der organischen Substanz in diesen Böden (dissert.) Hamburger Bodenkundliche Arbeiten 13: 1-228.

1990 Terminal report to the Deutsche Gesellschaft für Technische Zusammenarbeit (GTZ) on the project "International Agricultural Research. Measurement of natural ${ }^{14} \mathrm{C}$ concentrations in thin layers of soil profiles of Asia. Contract no. 72.7866.6-01.400/1420" and to the Deutsche Forschungsgemeinschaft (DFG) on the project "Carbon fluxes in important soil classes, with emphasis on Lessiver soils and on soils of the terrestrial, of the hydromorphic, and temporarily submerged environment. Contract Scha 47/23". Hamburg: 1-177.

(ms.) 1992 On-farm optimisation of the biological nitrogen fixation of grain legumes. Final report to the École Supérieure d'Agriculture d'Angers, March 1990-December 1992.

Becker-Heidmann, P. 1996 Requirements for an international radiocarbon soils database. Radiocarbon, this issue.
Becker-Heidmann, P., Lehfeldt, R. and Schipmann, R. 1995 Ein interaktives Simulationssystem zur Modellierung der Dynamik der organischen Substanz des Bodens. Mitteilungen der Deutschen Bodenkundlichen Gesellschaft 76: 733-736.

Becker-Heidmann, P., Liu, L.-W. and Scharpenseel, H.W. 1988 Radiocarbon dating of organic matter fractions of a Chinese Mollisol. Zeitschrift für Pflanzenernaehrung und Bodenkunde 151: 37-39.

Becker-Heidmann, P. and Scharpenseel, H. W. 1986 Thin layer $\delta^{13} \mathrm{C}$ and $\mathrm{D}^{14} \mathrm{C}$ monitoring of "Lessive" soil profiles. In Stuiver, M. and Kra, R., eds., Proceedings of the 12 th International ${ }^{14} \mathrm{C}$ Conference. Radiocarbon 28(2A): 383-390.

1989 Carbon isotope dynamics in some tropical soils. In Long, A., Kra, R. S. and Srdoc, D., eds., Proceedings of the 13 th International ${ }^{14} \mathrm{C}$ Conference. $R a$ diocarbon 31(3): 672-679.

1992a Studies of soil organic matter dynamics using natural carbon isotopes. The Science of the Total Environment 117/118: 305-312.

1992b The use of natural ${ }^{14} \mathrm{C}$ and ${ }^{13} \mathrm{C}$ in soils for studies on global climate change. In Long, A. and $\mathrm{Kra}$, R. S., eds., Proceedings of the 14 th International ${ }^{14} \mathrm{C}$ Conference. Radiocarbon 34(3): 535-540.

Becker-Heidmann, P. M., Martin, U. and Scharpenseel, H. W. 1985 Radiokohlenstoffdatierung und Abbau 
von ${ }^{14} \mathrm{C}$-markiertem Reisstroh zur Modellierung der Kohlenstoffdynamik eines Reisbodens. Mitteilungen der Deutschen Bodenkundlichen Gesellschaft 43(2): 525-530.

Bertram, H. G. 1986 Zur Rolle des Bodens im globalen Kohlenstoffzyklus. Messung der Temperaturabhängigkeit der Abbaurate des organischen Kohlenstoffs im Boden. Veröffentlichungen der Naturforschenden Gesellschaft zu Emden von 1814 (dissert.) 8.

Chang, J. M., Chen, Z. S., Chen, C. C. and Lin, C. F. 1983 Soil fertility characteristics of Taiwan paddy soils and their significance in soil numerical classification, (1) Alluvial soils in Kaohsiung-Pingtung region and latosols and lateritic alluvial soils in Taoyuan prefecture. Journal of the Agriculture Association of China 123: $50-68$.

Drachenberg, I. (ms.) 1992 Kennzeichnung des Humuszustandes von Böden unterschiedlicher Klimazonen mit Hilfe von Isotopenmethoden. Diploma thesis, Hamburg.

Gal, M., Amiel, A. J. and Ravikovitch, S. 1974 Clay mineral distribution and the origin in the soil types of Israel. Journal of Soil Science 25: 79-89.

Greenland, D. J. 1971 Interactions between humic and fulvic acids and clay. Soil Science 111: 34.

Harrison, K. G., Broecker, W. S. and Bonani, G. 1993 The effect of changing land use on soil radiocarbon. Science 262: 725-726.

Haupenthal, C., Scharpenseel, H. W., Eichwald, E. and Kirschey, K. G. 1979 Zum Einfluss einiger Standortfaktoren auf den Ertrag der Reispflanze in zwei Zinkmangel-Gebieten der Philippinen. Mitteilungen der Deutschen Bodenkundlichen Gesellschaft 29: 623632.

Martin, U. 1985 Decomposition of uniformly C14-labelled rice straw in a continuously flooded soil in the Philippines, Diss. Hamburger Bodenkundliche Arbeiten 6: 1-129.

Munsell Soil Color Charts 1975 Baltimore, Munsell Color.

Murthy, R .S., Hirekerur, L. R., Deshpande, S. B. and Venkata Rao, B. V., eds. 1982 Benchmark Soils of India. Bangalore, National Bureau of Soil Survey and Land Use Planning: $374 \mathrm{p}$.

Neue, H. U. (ms.) 1980 Methodischer Vergleich von Neutronentiefensonden anhand von Modelluntersuchungen und mehrjährigen Bodenfeuchtemessungen auf Löss-, Sandlöss- und Geschiebelehmstandorten. Dissertation, Hamburg: 1-285.

Neue, H. U. and Scharpenseel, H.W. 1987 Decomposition pattern of ${ }^{14} \mathrm{C}$-labeled rice straw in aerobic and submerged soils of the Philippines. Science of the Total Environment 62: 431-434.

Neue, H. U., Becker-Heidmann, P. and Scharpenseel, H. W. 1990 Organic matter dynamics, soil properties and cultural practices in rice lands and their relationship to methane production. In Bouwman, A. F., ed., Soils and the Greenhouse Effect. Proceedings of the International Conference on Soils and the Greenhouse Effect. Chichester, John Wiley \& Sons: 457-466.

O'Brien, B. J. and Stout, J. D. 1978 Movement and turnover of soil organic matter as indicated by carbon isotope measurements. Soil Biology and Biochemistry 10: 309-317.

Scharpenseel, H. W. 1978 Organo-mineralische Zinkfixierung in einer Reisbodencatena. Mitteilungen der Deutschen Bodenkundlichen Gesellschaft 27: 215220.

Scharpenseel, H. W. and Becker-Heidmann, P. 1989 Shifts in ${ }^{14} \mathrm{C}$-pattern of soil profiles due to bomb carbon, including effects of morphogenetic and turbation processes. In Long, A., Kra, R. S. and Srdoč, D., eds., Proceedings of the 13 th International ${ }^{14} \mathrm{C}$ Conference. Radiocarbon 31(3): 627-636.

1992 Twenty-five years of radiocarbon dating of soils: Paradigm of erring and learning. In Long, A. and Kra, R. S., eds., Proceedings of the 14th International ${ }^{14} \mathrm{C}$ Conference. Radiocarbon 34(3): 541-549.

1993 The dilemma of conflicting interests between $\mathrm{CO}_{2}$ 's and $\mathrm{CH}_{4}$ 's IR trapping capacity and role, in case of $\mathrm{CO}_{2}$ even as limiting factor, for plant growth. World Resource Review 4(2): 242-258.

1994a Sustainable land use in the light of resilience/ elasticity to soil organic matter fluctuations. In Greenland, D. and Szabolcs, I., eds., Soil Resilience and Sustainable Land Use. Proceedings of the Symposium, Budapest 28 September to 2 October 1992. Wallingford, CAB International: 249-264.

$1994 \mathrm{~b}{ }^{14} \mathrm{C}$ dates and ${ }^{13} \mathrm{C}$ measures of different soil species. In Lal, R., Kimble, J. M., and Levine, E., eds., Soil Processes and the Greenhouse Effect. Proceedings of the International Symposium on Greenhouse Gas Emissions and Carbon Sequestration, Ohio, 5-9 April 1993. Lincoln, Nebraska, USDA, Soil Conservation Service: $72-89$.

Scharpenseel, H. W., Becker-Heidmann, P., Neue, H. U., Tsutsuki, K. 1989 Bomb-carbon, ${ }^{14} \mathrm{C}$-dating and $\delta^{13} \mathrm{C}$ measurements as tracers of organic matter dynamics as well as of morphogenetic and turbation processes. The Science of the Total Environment 81/82: 99-110.

Scharpenseel, H. W., Eichwald, E., Haupenthal, C. and Neue, H. U. 1983 Zinc deficiency in a soil toposequence, grown to rice, at Tiaong, Quezon Province, Philippines. Catena 10: 115-132.

Scharpenseel, H. W., Pfeiffer, E.-M. and BeckerHeidmann, P. 1995a Organic carbon storage in tropical hydromorphic soils. In Carter, M. R. and Stewart, B. A., eds., Structure and Organic Matter Storage in Agricultural Soils. Boca Raton, Florida, CRC Lewis: 361-392.

1995b Soil organic matter studies and nutrient cycling. In Nuclear Techniques in Soil-Plant Studies for Sustainable Agriculture and Environmental Preservation. Proceedings of the International Symposium, 
17-21 October 1994. Vienna, IAEA: 285-305.

Scharpenseel, H. W. and Pietig, F. 1970 Altersbestimmung mit dem Flüssigkeits-Szintillations-Spektrometer - Vereinfachte Benzolsynthese, auch aus kleinen $\mathrm{CO}_{2}$-Mengen. Atompraxis 16(3): 1-2.

Scharpenseel, H. W., Tsutsuki, K., Becker-Heidmann, P. and Freytag, J. 1986 Untersuchungen zur Kohlenstoffdynamik und Bioturbation von Mollisolen. Zeitschrift für Pflanzenernaehrung und Bodenkunde 149: 582597.

Singer, S. 1993 The turnover of ${ }^{14} \mathrm{C}$ labelled groundnut straw, soil organic matter dynamics, and $\mathrm{CO}_{2}$ evolution in an Alfisol and a Vertisol of semi-arid tropical India (dissert.), Hamburger Bodenkundliche Arbeiten 19: 1-235.

Snitwongse, P., Phongpan, S. and Neue, H. U. (ms.) 1988 Decomposition of ${ }^{14} \mathrm{C}$-labelled rice straw in submerged and aerated rice soil in northeastern Thailand. Paper presented at the 1st International Symposium on Paddy Soil Fertility, Chiang Mai, Thailand, Dec. 613, 1988.

Stout, J. D., Goh, K. M. and Rafter, T. A. 1981 Chemistry and turnover of naturally occurring resistant organic compounds in soil. In Paul, E. A. and Ladd, J. N., eds., Soil Biochemistry. New York, Marcel Dekker: 1-73.

Stuiver, M. and Polach, H. A. 1977 Discussion: Reporting of ${ }^{14} \mathrm{C}$ data. Radiocarbon 19(3): 355-363.

Theng, B. K. G. 1979 Formation and Properties of Clay Polymer Complexes. London, Hilger: 362 p.

van Breemen, N. 1976 Genesis and Solution Chemistry of Acid Sulfate Soils in Thailand. Wageningen, Center for Agricultural Publishing and Documentation: 263 p.

van der Kevie, W. 1972 Morphology, genesis, occurrence and agricultural potential of acid sulfate soils in Central Thailand. Thai Journal of Agricultural Science: 165-182.

Yaalon, D. H. and Kalmar, D. 1972 Vertical movement in an undisturbed soil: Continuous measurement of swelling and shrinkage with a sensitive apparatus. Geoderma 8: 231-240.

1978 Dynamics of cracking and swelling clay soils: Displacement of skeletal grains, optimum depth of slickensides, and rate of intra-pedonic turbation. Earth Surface Processes 3: 31-42.

\section{Ohlendorf Forest, Profile A}

\section{Profile-Related Data}

Location (longitude, latitude):

Location (country, next city or village):

Soil order and type (FAO classification):

Soil order and type (local classification):

Parent material:

Mean annual temperature:

Annual rainfall:

Vegetation and land use:

Site description:

Date of sampling:

Date of measurement:

Collector:

Submitter:

Submitter's comment:

Literature references:

$52^{\circ} 17^{\prime} \mathrm{N}, 9^{\circ} 42^{\prime} \mathrm{E}$

Germany, Hiddestorf south of Hannover

Stagnic Luvisol

Pseudogley-Parabraunerde

Würmian loess

$8.5^{\circ} \mathrm{C}$

$620 \mathrm{~mm}$

120-yr-old beech stand (Fagus sylvatica L.) with single Carpinus betulus L. and oaks (Quercus robur

L.), farmer's woodland

$67.0 \mathrm{~m}$ above sea level (asl), plane

1982

1983 and 1984

Becker-Heidmann

Becker-Heidmann

See literature refs.

Becker-Heidmann (1989); Becker-Heidmann and

Scharpenseel (1986); Neue (1980) 
Description of Profile A at Ohlendorf Forest

\begin{tabular}{|c|c|c|}
\hline Depth (cm) & Horizon & Description \\
\hline-4 to -1 & $\mathrm{~L}$ & Litter \\
\hline-1 to 0 & Of & \\
\hline $0-2$ & Ah1 & Brown $(10 \mathrm{YR} 5 / 3)^{1}$, silt, roots \\
\hline $2-4$ & Ah2 & Silt loam \\
\hline $4-8$ & $\mathrm{Ae}$ & White (10YR 8/2), silt, few roots \\
\hline $8-15$ & Bhs & Humus enriched, light gray (10YR $7 / 2)$, silt loam, frequent roots \\
\hline $15-40$ & Al1 & $\begin{array}{l}\text { Clay eluviated, Lessivé, light gray (10YR } 7 / 2) \text {, silt loam, few to frequent } \\
\text { roots }\end{array}$ \\
\hline $40-50$ & $\mathrm{~A} 12$ & Pale brown (10YR 7/3), silt loam \\
\hline $50-60$ & SwBt1 & $\begin{array}{l}\text { Clay enriched, pale brown (10YR 7/4), silt loam, few coarse roots, clay } \\
\text { cutanes, few manganese concretions }\end{array}$ \\
\hline $60-70$ & SwBt2 & Pale brown (10YR 7/4), silt loam \\
\hline $70-78$ & $\mathrm{SdBt}$ & $\begin{array}{l}\text { Water impeding, clay enriched, pale brown (10YR 7/4), stark lehmiger } \\
\text { Schluff, manganese concretions }\end{array}$ \\
\hline
\end{tabular}

TABLE 1. Ohlendorf Forest, Profile A

\begin{tabular}{lccccrr}
\hline Lab code & Depth $(\mathrm{cm})$ & $\mathrm{pH}$ & $\mathrm{C}$ & $\delta^{13} \mathrm{C}$ & \multicolumn{1}{c}{${ }^{14} \mathrm{C}$ activity } & \multicolumn{1}{c}{${ }^{14} \mathrm{C}$ age } \\
\hline HAM-1652 & $0-2$ & -- & 5.05 & -28.0 & $113.33 \pm 0.74$ & Modern \\
HAM-1654 & $2-4$ & 3.6 & 2.23 & -27.4 & $99.20 \pm 1.02$ & $30 \pm 80$ \\
HAM-1655 & $4-6$ & 3.5 & 0.50 & -26.8 & $99.79 \pm 1.03$ & $30 \pm 80$ \\
HAM-1656 & $6-8$ & 3.7 & 0.53 & -27.1 & $94.50 \pm 0.99$ & $420 \pm 80$ \\
HAM-1657 & $8-10$ & 3.6 & 1.02 & -27.0 & $94.76 \pm 0.99$ & $400 \pm 80$ \\
HAM-1658 & $10-12$ & 3.7 & 0.76 & -27.0 & $96.48 \pm 1.00$ & $260 \pm 80$ \\
HAM-1659 & $12-15$ & 3.8 & 1.01 & -26.6 & $94.07 \pm 0.99$ & $470 \pm 90$ \\
HAM-1660 & $15-18$ & 3.8 & 0.72 & -27.0 & $94.45 \pm 0.98$ & $430 \pm 80$ \\
HAM-1661 & $18-20$ & 3.8 & 0.67 & -26.7 & $96.66 \pm 1.00$ & $250 \pm 80$ \\
HAM-1662 & $20-22$ & 3.8 & 0.53 & -26.7 & $96.70 \pm 0.87$ & $240 \pm 70$ \\
HAM-1663 & $22-24$ & 3.8 & 0.60 & -26.6 & $96.48 \pm 0.86$ & $260 \pm 70$ \\
HAM-1664 & $24-26$ & 3.8 & 0.47 & -26.8 & $99.15 \pm 0.89$ & $40 \pm 70$ \\
HAM-1665 & $26-28$ & 3.8 & 0.50 & -27.0 & $93.69 \pm 0.63$ & $490 \pm 50$ \\
HAM-1666 & $28-30$ & 3.8 & 0.53 & -26.4 & $93.61 \pm 0.98$ & $510 \pm 80$ \\
HAM-1667 & $30-32$ & 3.8 & 0.44 & -27.0 & $94.09 \pm 0.85$ & $460 \pm 70$ \\
HAM-1668 & $32-34$ & 3.8 & 0.45 & -26.0 & $91.62 \pm 0.84$ & $690 \pm 70$ \\
HAM-1669 & $34-36$ & 3.7 & 0.41 & -26.6 & $94.31 \pm 0.85$ & $440 \pm 70$ \\
HAM-1670 & $36-38$ & 3.8 & 0.33 & -26.3 & $96.32 \pm 0.97$ & $280 \pm 80$ \\
HAM-1671 & $38-40$ & 3.7 & 0.44 & -25.8 & $108.67 \pm 0.70$ & Modern \\
HAM-1672 & $40-42$ & 3.8 & 0.36 & -26.7 & $92.39 \pm 0.63$ & $610 \pm 60$ \\
HAM-1673 & $42-44$ & 3.7 & 0.37 & -26.4 & $94.42 \pm 0.64$ & $440 \pm 50$ \\
HAM-1674 & $44-46$ & 3.8 & 0.24 & -26.3 & $93.94 \pm 0.64$ & $480 \pm 50$ \\
HAM-1675 & $46-48$ & 3.8 & 0.27 & -26.4 & $89.76 \pm 0.94$ & $850 \pm 80$ \\
HAM-1676 & $48-50$ & 3.7 & 0.25 & -26.4 & $90.30 \pm 0.94$ & $800 \pm 80$ \\
HAM-1677 & $50-52$ & 3.7 & 0.22 & -26.3 & $86.90 \pm 0.92$ & $1110 \pm 90$ \\
HAM-1678 & $52-54$ & 3.8 & 0.19 & -26.0 & $86.62 \pm 0.90$ & $1140 \pm 80$ \\
HAM-1679 & $54-56$ & 3.7 & 0.20 & -25.9 & $83.52 \pm 0.88$ & $1430 \pm 90$
\end{tabular}

${ }^{1}$ For YR soil color codes, see Munsell Soil Color Charts (1975). 
TABLE 1. Ohlendorf Forest, Profile A (Continued)

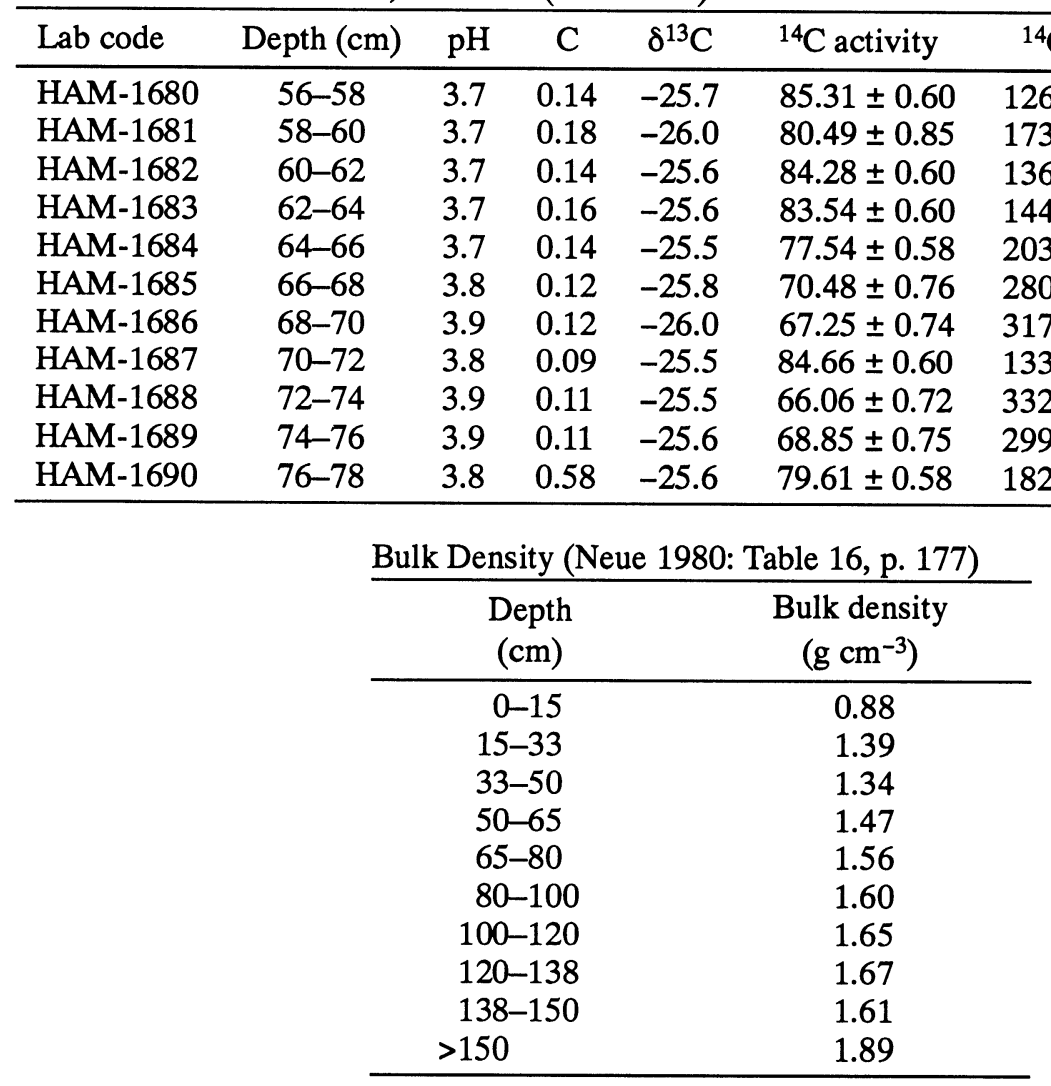

\section{Ohlendorf Forest, Profile B}

\section{Profile-Related Data}

Location (longitude, latitude):

Location (country, next city or village):

Soil order and type (FAO classification):

Soil order and type (local classification):

Parent material:

Mean annual temperature:

Annual rainfall:

Vegetation and land use:

Site description:

Date of sampling:

Date of measurement:

Collector:

Submitter:

Submitter's comment:

Literature references: $52^{\circ} 17^{\prime} \mathrm{N}, 9^{\circ} 42^{\prime} \mathrm{E}$

Germany, Hiddestorf, south of Hannover

Stagnic Luvisol

Parabraunerde-Pseudogley

Würmian loess

$8.5^{\circ} \mathrm{C}$

$620 \mathrm{~mm}$

120-yr-old beech stand (Fagus sylvatica $\mathrm{L}$.) with single Carpinus betulus L. and oaks (Quercus robur

L.), farmer's woodland

$66.2 \mathrm{~m}$ asl, plane, $c a$. $20 \mathrm{~m}$ southeast of Profile A

1983

1984 and 1985

Becker-Heidmann

Becker-Heidmann

See literature references

Becker-Heidmann (1989); Becker-Heidmann and

Scharpenseel (1986); Neue (1980) 


\section{Description of Profile B at Ohlendorf Forest}

\begin{tabular}{|c|c|c|}
\hline Depth (cm) & Horizon & Description \\
\hline-4 to 2 & $\mathrm{~L}$ & Litter \\
\hline-2 to 0 & Of & Fermented litter \\
\hline $0-5$ & $\mathrm{Ah}$ & Dark brown (10YR 4/3), silt loam, frequent roots \\
\hline $5-10$ & $\mathrm{Ae}$ & Pale brown (10YR 6/3), silt, few roots \\
\hline $10-20$ & Bhs & Light yellowish brown (10YR 6/4), silt loam, frequent roots \\
\hline $20-38$ & Al1 & Clay eluviated, Lessive, brownish yellow (10YR 6/6), silt, frequent roots \\
\hline $38-44$ & $\mathrm{~A} 12$ & Silt \\
\hline $44-58$ & SwAl & $\begin{array}{l}\text { Light yellowish brown (10YR 6/4), pale mottles, silt, few roots, esp. at } \\
\text { the lower border of the horizon }\end{array}$ \\
\hline $58-84$ & $\mathrm{SdBt}$ & $\begin{array}{l}\text { Yellowish brown (10YR } 5 / 6 \text { ) with dark mottles (clay lentils), silty clay } \\
\text { loam, in the upper part horizontal root veins, partly filled with Al mate- } \\
\text { rial, clay cutanes, few manganese concretions }\end{array}$ \\
\hline $84-90$ & BtSd1 & $\begin{array}{l}\text { Yellowish brown (10YR 5/6), more frequently mottled, silt loam, } \\
\text { increasing concretions }\end{array}$ \\
\hline 90-102 & $\mathrm{BtSd} 2$ & Yellowish brown (10YR 5/8), silt loam \\
\hline $102-140$ & $\mathrm{BtSd} 3$ & $\begin{array}{l}\text { Yellowish brown (10YR } 5 / 6) \text {, few roots, partly dead, gray lentil with fer- } \\
\text { rous coating, gravels partly with manganese coating, silt loam }\end{array}$ \\
\hline $140+$ & IIC & $\begin{array}{l}\text { Yellowish brown (10YR 5/6) to brown (10YR } 5 / 3) \text { mottled, gravelled } \\
\text { loam }\end{array}$ \\
\hline
\end{tabular}

TABLE 2. Ohlendorf Forest, Profile B

\begin{tabular}{ccccccc}
\hline Lab code & Depth $(\mathrm{cm})$ & $\mathrm{pH}$ & $\mathrm{C}$ & $\delta^{13} \mathrm{C}$ & ${ }^{14} \mathrm{C}$ activity & ${ }^{14} \mathrm{C}$ age \\
\hline HAM-1910 & $0-3$ & 3.8 & 5.42 & -26.7 & $107.22 \pm 0.72$ & Modern \\
HAM-1911 & $3-5$ & 3.6 & 5.17 & -26.4 & $100.23 \pm 0.71$ & Modern \\
HAM-1912 & $5-7$ & 3.8 & 2.10 & -26.1 & $99.52 \pm 0.68$ & $20 \pm 60$ \\
HAM-1913 & $7-10$ & 3.9 & 1.01 & -26.5 & $96.91 \pm 0.70$ & $230 \pm 60$ \\
HAM-1914 & $10-12$ & 3.9 & 1.45 & -26.4 & $95.48 \pm 0.67$ & $350 \pm 60$ \\
HAM-1915 & $12-14$ & 4.0 & 1.28 & -26.5 & $98.37 \pm 0.68$ & $110 \pm 60$ \\
HAM-1916 & $14-16$ & 4.1 & 1.41 & -26.6 & $96.48 \pm 0.67$ & $260 \pm 60$ \\
HAM-1917 & $16-18$ & 3.8 & 1.02 & -26.3 & $97.74 \pm 0.67$ & $160 \pm 60$ \\
HAM-1918 & $18-20$ & 3.8 & 1.36 & -26.3 & $95.82 \pm 0.67$ & $320 \pm 60$ \\
HAM-1919 & $20-22$ & 3.8 & 0.73 & -26.0 & $97.09 \pm 0.67$ & $220 \pm 60$ \\
HAM-1920 & $22-24$ & 3.8 & 0.59 & -26.0 & $95.00 \pm 0.74$ & $400 \pm 60$ \\
HAM-1921 & $24-26$ & 3.8 & 0.58 & -26.3 & $95.18 \pm 0.66$ & $380 \pm 60$ \\
HAM-1922 & $26-28$ & 4.0 & 0.39 & -25.9 & $95.51 \pm 0.67$ & $360 \pm 60$ \\
HAM-1923 & $28-30$ & 3.9 & 0.40 & -25.5 & $93.20 \pm 0.66$ & $560 \pm 60$ \\
HAM-1924 & $30-32$ & 3.8 & 0.32 & -25.5 & $90.69 \pm 0.66$ & $780 \pm 60$ \\
HAM-1925 & $32-34$ & 3.8 & 0.31 & -25.2 & $91.03 \pm 0.65$ & $750 \pm 60$ \\
HAM-1926 & $34-36$ & 3.8 & 0.34 & -25.3 & $89.03 \pm 0.64$ & $930 \pm 60$ \\
HAM-1927 & $36-38$ & 3.8 & 0.16 & -25.1 & $96.47 \pm 0.67$ & $290 \pm 60$ \\
HAM-1928 & $38-40$ & 3.9 & 0.20 & -25.1 & $87.29 \pm 0.63$ & $1090 \pm 60$ \\
HAM-1929 & $40-42$ & 3.8 & 0.23 & -25.0 & $86.49 \pm 0.63$ & $1170 \pm 60$ \\
HAM-1930 & $42-44$ & 3.9 & 0.16 & -25.3 & $84.25 \pm 0.62$ & $1370 \pm 60$ \\
HAM-1931 & $44-46$ & 3.9 & 0.12 & -25.1 & $94.57 \pm 0.67$ & $450 \pm 60$ \\
HAM-1932 & $46-48$ & 3.8 & 0.15 & -25.2 & $86.61 \pm 0.63$ & $1150 \pm 60$ \\
HAM-1933 & $48-50$ & 3.8 & 0.12 & -25.1 & $87.39 \pm 0.64$ & $1080 \pm 60$
\end{tabular}


TABLE 2. Ohlendorf Forest, Profile B (Continued)

\begin{tabular}{|c|c|c|c|c|c|c|}
\hline Lab code & Depth (cm) & $\mathrm{pH}$ & $\mathrm{C}$ & $\delta^{13} \mathrm{C}$ & ${ }^{14} \mathrm{C}$ activity & ${ }^{14} \mathrm{C}$ age \\
\hline HAM-1934 & $50-52$ & 3.8 & 0.13 & -25.3 & $74.58 \pm 0.59$ & $2350 \pm 60$ \\
\hline HAM-1935 & $52-54$ & 3.7 & 0.14 & -25.2 & $71.09 \pm 0.57$ & $2740 \pm 70$ \\
\hline HAM-1936 & $54-56$ & 3.5 & 0.15 & -25.0 & $82.75 \pm 0.62$ & $1520 \pm 60$ \\
\hline HAM-1937 & $56-58$ & 3.5 & 0.17 & -24.6 & $95.76 \pm 0.67$ & $350 \pm 60$ \\
\hline HAM-1938 & $58-60$ & 3.5 & 0.17 & -24.7 & $85.45 \pm 0.59$ & $1270 \pm 60$ \\
\hline HAM-1939 & $60-62$ & 3.5 & 0.20 & -24.4 & $87.76 \pm 0.64$ & $1060 \pm 60$ \\
\hline HAM-1940 & $62-64$ & 3.6 & 0.18 & -24.4 & $88.21 \pm 0.64$ & $1020 \pm 60$ \\
\hline HAM-1941 & $64-66$ & 3.6 & 0.19 & -24.3 & $75.85 \pm 0.59$ & $2230 \pm 60$ \\
\hline HAM-1942 & $66-68$ & 3.7 & 0.15 & -24.7 & $90.02 \pm 0.65$ & $850 \pm 60$ \\
\hline HAM-1943 & $68-70$ & 3.8 & 0.17 & -24.8 & $82.29 \pm 0.68$ & $1570 \pm 70$ \\
\hline HAM-1944 & $70-72$ & 3.6 & 0.16 & -24.5 & $84.23 \pm 0.68$ & $1390 \pm 60$ \\
\hline HAM-1945 & $72-74$ & 3.8 & 0.13 & -24.5 & $78.79 \pm 0.66$ & $1920 \pm 70$ \\
\hline HAM-1946 & $74-76$ & 4.0 & 0.11 & -24.4 & $83.34 \pm 0.67$ & $1470 \pm 70$ \\
\hline HAM-1947 & $76-78$ & 3.9 & 0.10 & -24.4 & $72.82 \pm 0.64$ & $2560 \pm 70$ \\
\hline HAM-1948 & $78-80$ & 4.1 & 0.09 & -24.5 & $85.76 \pm 0.68$ & $1240 \pm 60$ \\
\hline HAM-1949 & $80-82$ & 4.1 & 0.08 & -24.4 & -- & -- \\
\hline HAM-1950 & $82-84$ & 4.2 & 0.07 & -24.5 & $111.16 \pm 0.80$ & Modern \\
\hline HAM-1951 & $84-86$ & 4.0 & 0.10 & -24.2 & $83.09 \pm 0.68$ & $1500 \pm 70$ \\
\hline HAM-1952 & $86-88$ & 4.3 & 0.08 & -24.6 & $88.24 \pm 0.80$ & $1010 \pm 70$ \\
\hline HAM-1953 & $88-90$ & 4.2 & 0.09 & -24.5 & $113.45 \pm 0.80$ & Modern \\
\hline HAM-1954 & $90-93$ & 4.5 & 0.08 & -24.1 & $93.04 \pm 0.73$ & $590 \pm 60$ \\
\hline HAM-1955 & $93-96$ & 4.3 & 0.09 & -24.4 & $85.60 \pm 0.68$ & $1260 \pm 60$ \\
\hline HAM-1956 & 96-99 & 4.6 & 0.08 & -24.1 & $73.02 \pm 0.63$ & $2540 \pm 70$ \\
\hline HAM-1957 & 99-102 & 4.6 & 0.07 & -24.7 & $123.09 \pm 0.84$ & Modern \\
\hline HAM-1958 & $102-105$ & 4.7 & 0.07 & -24.6 & $95.13 \pm 0.72$ & $410 \pm 60$ \\
\hline HAM-1959 & $105-108$ & 4.7 & 0.06 & -24.4 & $96.23 \pm 0.73$ & $320 \pm 60$ \\
\hline HAM-1960 & $108-111$ & 4.9 & 0.09 & -24.5 & $92.65 \pm 0.71$ & $620 \pm 60$ \\
\hline HAM-1961 & $111-114$ & 4.9 & 0.08 & -24.2 & $71.27 \pm 0.70$ & $2730 \pm 80$ \\
\hline HAM-1962 & $114-117$ & 5.1 & 0.07 & -24.5 & $81.21 \pm 0.85$ & $1680 \pm 80$ \\
\hline HAM-1963 & $117-120$ & 5.1 & 0.11 & -24.0 & $75.63 \pm 0.87$ & $2260 \pm 90$ \\
\hline HAM-1964 & $120-123$ & 5.7 & 0.11 & -25.4 & $81.23 \pm 0.67$ & $1660 \pm 70$ \\
\hline HAM-1965 & $123-126$ & 5.7 & 0.07 & -24.7 & $73.77 \pm 0.63$ & $2450 \pm 70$ \\
\hline HAM-1966 & $126-129$ & 5.8 & 0.06 & -25.7 & $85.46 \pm 0.68$ & $1250 \pm 60$ \\
\hline HAM-1967 & $129-132$ & 6.2 & 0.07 & -25.4 & $76.29 \pm 0.64$ & $2170 \pm 70$ \\
\hline HAM-1968 & $132-135$ & 6.0 & 0.06 & -25.6 & $73.61 \pm 0.63$ & $2450 \pm 70$ \\
\hline HAM-1969 & $135-138$ & 6.0 & 0.06 & -25.6 & $84.84 \pm 0.68$ & $1310 \pm 60$ \\
\hline HAM-1970 & $138-140$ & 5.9 & 0.06 & -25.0 & $84.48 \pm 0.79$ & $1350 \pm 80$ \\
\hline HAM-1971 & $140-142$ & 5.8 & 0.06 & -25.2 & $77.71 \pm 0.73$ & $2020 \pm 80$ \\
\hline HAM-1972 & $142-144$ & 6.0 & 0.06 & -24.9 & $96.02 \pm 0.90$ & $330 \pm 80$ \\
\hline HAM-1973 & $144-146$ & 5.9 & 0.10 & -26.4 & $98.01 \pm 0.74$ & $140 \pm 60$ \\
\hline
\end{tabular}

Bulk Density (Neue 1980: Table 17, p. 178)

\begin{tabular}{cc}
\hline $\begin{array}{c}\text { Depth } \\
(\mathrm{cm})\end{array}$ & $\begin{array}{c}\text { Bulk density } \\
\left(\mathrm{g} \mathrm{cm}^{-3}\right)\end{array}$ \\
\hline $0-5$ & 0.92 \\
$5-10$ & 1.23 \\
$10-20$ & 1.30 \\
$20-37$ & 1.37
\end{tabular}


Bulk Density (Neue 1980: Table 17, p. 178) (Continued)

\begin{tabular}{cc}
\hline $\begin{array}{c}\text { Depth } \\
(\mathrm{cm})\end{array}$ & $\begin{array}{c}\text { Bulk density } \\
\left(\mathrm{g} \mathrm{cm}^{-3}\right)\end{array}$ \\
\hline $37-50$ & 1.48 \\
$50-70$ & 1.52 \\
$70-110$ & 1.59 \\
$110-130$ & 1.66 \\
$130-144$ & 1.72 \\
$144-164$ & 1.85 \\
\hline
\end{tabular}

\section{WOHLDORF FOREST}

\section{Profile-Related Data}

Location (longitude, latitude):

Location (country, next city or village):

Soil order and type (FAO classification):

Soil order and type (local classification):

Parent material:

Mean annual temperature:

Annual rainfall:

Vegetation and land use:

Site description:

Date of sampling:

Date of measurement:

Collector:

Submitter:

Submitter's comment:

Literature references:

\author{
$53^{\circ} 43^{\prime} \mathrm{N}, 10^{\circ} 9^{\prime} \mathrm{E}$ \\ Germany, Hamburg \\ Stagnic Luvisol \\ Podsolige Braunerde above Parabraunerde-Pseudog- \\ ley \\ Weichselian boulder cover sand above Saalean boul- \\ der loam \\ $8.5^{\circ} \mathrm{C}$ \\ $741 \mathrm{~mm}$ \\ 100 -yr beech stand, forest, nature reserve \\ $31.1 \mathrm{~m}$ asl, top of terminal moraine, plane \\ 1982 \\ 1983 \\ Becker-Heidmann \\ Becker-Heidmann \\ See literature references \\ Becker-Heidmann (1989); Becker-Heidmann and \\ Scharpenseel (1986); Scharpenseel and Becker- \\ Heidmann (1994b)
}

\section{Description of the Profile at Wohldorf Forest}

\begin{tabular}{|c|c|c|}
\hline Depth $(\mathrm{cm})$ & Horizon & Description \\
\hline-8 to 5 & $\mathrm{~L}$ & Litter \\
\hline-5 to 3 & Of1 & Leaf structure visible \\
\hline-3 to 1 & Of2 & Macerated litter \\
\hline-1 to 0 & $\mathrm{Oh}$ & \\
\hline $0-3$ & Ah & Grayish brown (10YR 5/2), loamy sand, very frequent roots \\
\hline $3-5$ & $\mathrm{Ae}$ & Light gray (10YR $7 / 2)$, loamy sand, few roots \\
\hline $5-11$ & Bhs & Very pale brown (10YR $7 / 3)$, sandy loam, very frequent roots \\
\hline $11-23$ & $\mathrm{Bv}$ & Very pale brown (10YR $7 / 3)$, sandy loam, very frequent roots \\
\hline $23-40$ & IISwAl & Clay eluviated (Lessivé), white (10YR $8 / 2$ ), sandy loam, few roots \\
\hline $40-50$ & IISdBt1 & $\begin{array}{l}\text { Clay enriched, water impeding, very pale brown (10YR 8/3), sandy } \\
\text { loam, few roots }\end{array}$ \\
\hline $50-63$ & IISdBt2 & Very pale brown (10YR 8/4), sandy loam \\
\hline
\end{tabular}


63-73 IISdBt3

73-88 IISdBt4

88-100 IISdBt5
Very pale brown (10YR 8/4), silt loam, roots in leading channels

Very pale brown (10YR 8/4), loam, roots in leading channels

Very pale brown (10YR 7/4), mottled, sandy loam, roots in leading channels

100-110+ IIICv Very pale brown (10YR 7/4), loamy sand, gravels, bands, iron enriched

TABLE 3. Wohldorf Forest

\begin{tabular}{|c|c|c|c|c|c|c|}
\hline Lab code & Depth $(\mathrm{cm})$ & $\mathrm{pH}$ & C & $\delta^{13} \mathrm{C}$ & ${ }^{14} \mathrm{C}$ activity & ${ }^{14} \mathrm{C}$ age \\
\hline HAM-1750 & -8 to -5 & -- & 40.36 & -30.4 & -- & -- \\
\hline HAM-1751 & -5 to -4 & -- & 27.38 & -30.2 & $129.23 \pm 0.87$ & Modern \\
\hline HAM-1752 & -4 to -3 & -- & 29.33 & -30.0 & $142.47 \pm 0.92$ & Modern \\
\hline HAM-1753 & -3 to -1 & -- & 25.89 & -27.4 & $145.06 \pm 0.94$ & Modern \\
\hline HAM-1754 & -1 to 0 & -- & 15.77 & -27.1 & $107.05 \pm 0.77$ & Modern \\
\hline HAM-1755 & $0-3$ & 3.0 & 3.46 & -28.6 & $103.26 \pm 0.88$ & Modern \\
\hline HAM-1756 & $3-5$ & 3.4 & 0.72 & -28.1 & $113.27 \pm 0.95$ & Modern \\
\hline HAM-1757 & $5-7$ & 3.7 & 1.36 & -27.5 & $107.18 \pm 1.03$ & Modern \\
\hline HAM-1758 & $7-9$ & 3.7 & 1.26 & -27.1 & $105.00 \pm 1.01$ & Modern \\
\hline HAM-1759 & 9-11 & 3.8 & 1.46 & -27.8 & $102.44 \pm 0.99$ & Modern \\
\hline HAM-1760 & $11-13$ & 3.9 & 0.75 & -27.3 & $97.93 \pm 0.85$ & $130 \pm 70$ \\
\hline HAM-1761 & $13-15$ & 4.0 & 0.88 & -27.3 & $116.07 \pm 1.01$ & Modern \\
\hline HAM-1762 & $15-17$ & 3.9 & 0.99 & -27.4 & $95.51 \pm 0.83$ & $330 \pm 70$ \\
\hline HAM-1763 & $17-19$ & 4.1 & 1.13 & -27.1 & $95.37 \pm 0.83$ & $350 \pm 70$ \\
\hline HAM-1764 & $19-21$ & 4.1 & 0.45 & -26.9 & $96.69 \pm 0.84$ & $240 \pm 70$ \\
\hline HAM-1765 & $21-23$ & 4.1 & 0.26 & -27.1 & $95.08 \pm 0.83$ & $370 \pm 70$ \\
\hline HAM-1766 & $23-25$ & 4.1 & 0.78 & -27.5 & $106.75 \pm 1.03$ & Modern \\
\hline HAM-1767 & $25-27$ & 4.2 & 0.47 & -27.3 & $125.62 \pm 1.17$ & Modern \\
\hline HAM-1768 & $27-29$ & 4.2 & 0.46 & -27.2 & $97.96 \pm 0.85$ & $130 \pm 70$ \\
\hline HAM-1769 & $29-31$ & 4.2 & 1.29 & -27.4 & $94.84 \pm 0.83$ & $390 \pm 70$ \\
\hline HAM-1770 & $31-33$ & 4.2 & 0.53 & -27.2 & $93.50 \pm 0.82$ & $510 \pm 70$ \\
\hline HAM-1771 & $33-35$ & 4.3 & 0.21 & -27.0 & $99.24 \pm 1.09$ & $30 \pm 90$ \\
\hline HAM-1772 & $35-37$ & 4.2 & 0.38 & -27.1 & $101.49 \pm 1.00$ & Modern \\
\hline HAM-1773 & $37-40$ & 4.2 & 0.16 & -26.7 & $114.98 \pm 1.10$ & Modern \\
\hline HAM-1774 & $40-42$ & 4.2 & 0.16 & -26.4 & $119.28 \pm 1.14$ & Modern \\
\hline HAM-1775 & $42-44$ & 4.1 & 0.14 & -26.4 & $110.81 \pm 1.05$ & Modern \\
\hline HAM-1776 & $44-46$ & 4.1 & 0.17 & -25.7 & $89.87 \pm 0.80$ & $850 \pm 70$ \\
\hline HAM-1777 & $46-48$ & 4.0 & 0.16 & -26.2 & $88.57 \pm 1.10$ & $960 \pm 100$ \\
\hline HAM-1778 & $48-50$ & 4.0 & 0.15 & -25.9 & $103.67 \pm 1.00$ & Modern \\
\hline HAM-1779 & $50-52$ & 3.9 & 0.15 & -25.8 & $94.06 \pm 0.91$ & $480 \pm 80$ \\
\hline HAM-1780 & $52-55$ & 3.9 & 0.10 & -25.7 & $87.14 \pm 0.78$ & $1090 \pm 70$ \\
\hline HAM-1781 & $55-58$ & 3.8 & 0.15 & -25.5 & $89.13 \pm 0.79$ & $920 \pm 70$ \\
\hline HAM-1782 & $58-60$ & 3.9 & 0.07 & -26.1 & $78.49 \pm 0.72$ & $1930 \pm 70$ \\
\hline HAM-1783 & $60-63$ & 3.9 & 0.11 & -26.1 & $94.90 \pm 0.92$ & $400 \pm 80$ \\
\hline HAM-1784 & $63-65$ & 3.8 & 0.12 & -25.7 & $94.67 \pm 0.92$ & $430 \pm 80$ \\
\hline HAM-1785 & $65-67$ & 3.8 & 0.19 & -25.7 & $83.23 \pm 0.76$ & $1460 \pm 70$ \\
\hline HAM-1786 & $67-69$ & 3.8 & 0.10 & -25.4 & $82.14 \pm 0.76$ & $1570 \pm 70$ \\
\hline HAM-1787 & $69-71$ & 3.8 & 0.14 & -25.4 & $86.24 \pm 0.78$ & $1180 \pm 70$ \\
\hline HAM-1788 & $71-73$ & 3.8 & 0.10 & -25.7 & $92.33 \pm 0.92$ & $630 \pm 80$ \\
\hline HAM-1789 & $73-75$ & 3.8 & 0.09 & -25.9 & $85.78 \pm 0.85$ & $1220 \pm 80$ \\
\hline HAM-1790 & $75-77$ & 3.8 & 0.09 & -25.7 & $85.11 \pm 0.85$ & $1280 \pm 80$ \\
\hline HAM-1791 & $77-80$ & 3.8 & 0.07 & -26.1 & $79.68 \pm 0.80$ & $1810 \pm 80$ \\
\hline HAM-1792 & $80-82$ & 3.8 & 0.10 & -25.7 & $77.75 \pm 0.79$ & $2010 \pm 80$ \\
\hline HAM-1793 & $82-84$ & 3.7 & 0.09 & -25.3 & $74.73 \pm 0.86$ & $2340 \pm 90$ \\
\hline
\end{tabular}


TABLE 3. Wohldorf Forest (Continued)

\begin{tabular}{ccccccc}
\hline Lab code & Depth $(\mathrm{cm})$ & $\mathrm{pH}$ & $\mathrm{C}$ & $\delta^{13} \mathrm{C}$ & ${ }^{14} \mathrm{C}$ activity & ${ }^{14} \mathrm{C}$ age \\
\hline HAM-1794 & $84-86$ & 3.8 & 0.09 & -26.2 & $76.78 \pm 0.71$ & $2100 \pm 80$ \\
HAM-1795 & $86-88$ & 3.8 & 0.08 & -26.3 & $81.92 \pm 0.75$ & $1580 \pm 70$ \\
HAM-1796 & $88-90$ & 3.7 & 0.06 & -26.1 & $77.29 \pm 0.73$ & $2050 \pm 80$ \\
HAM-1797 & $90-92$ & 3.7 & 0.05 & -25.4 & $74.92 \pm 0.70$ & $2310 \pm 80$ \\
HAM-1798 & $92-94$ & 3.6 & 0.07 & -25.0 & $85.60 \pm 0.84$ & $1250 \pm 90$ \\
HAM-1799 & $94-96$ & 3.7 & 0.06 & -25.6 & -- & - \\
HAM-1800 & $96-98$ & 3.7 & 0.06 & -24.9 & $78.95 \pm 0.73$ & $1900 \pm 70$ \\
HAM-1801 & $98-100$ & 3.7 & 0.04 & -26.8 & $82.78 \pm 0.87$ & $1490 \pm 80$ \\
HAM-1802 & $100-102$ & 3.7 & 0.06 & -26.2 & $99.16 \pm 0.88$ & $50 \pm 70$ \\
HAM-1803 & $102-104$ & 3.8 & 0.05 & -25.7 & $94.10 \pm 0.79$ & $480 \pm 70$ \\
HAM-1804 & $104-106$ & 3.8 & 0.04 & -27.6 & $84.71 \pm 0.72$ & $1290 \pm 70$ \\
HAM-1805 & $106-108$ & 3.9 & 0.04 & -28.8 & $79.20 \pm 0.93$ & $1810 \pm 90$ \\
HAM-1806 & $108-110$ & 4.0 & 0.03 & -27.4 & $80.96 \pm 1.14$ & $1660 \pm 110$ \\
\hline
\end{tabular}

TIMMENDORF FOREST

\section{Profile-Related Data}

Location (longitude, latitude):

Location (country, next city or village):

Soil order and type (FAO classification):

Soil order and type (local classification):

Parent material:

Mean annual temperature:

Annual rainfall:

Vegetation and land use:

Site description:

Date of sampling:

Date of measurement:

Collector:

Submitter:

Submitter's comment:

Lab comment:

Literature references: $54^{\circ} 0^{\prime} \mathrm{N}, 10^{\circ} 47^{\prime} \mathrm{E}$

Germany, Timmendorf

Stagnic Luvisol

Pseudogley-Parabraunerde

Weichselian loam

$8.2^{\circ} \mathrm{C}$

$600 \mathrm{~mm}$

110-yr-old beech (Fagus sylvatica L.), nature reserve $2.5 \mathrm{~m}$ asl, plane

1982

1984

Becker-Heidmann

Becker-Heidmann

See literature references

$-$.

Becker-Heidmann (1989)

\section{Description of the Profile at Timmendorf Forest}

\begin{tabular}{|c|c|c|}
\hline Depth $(\mathrm{cm})$ & Horizon & Description \\
\hline-4 to -3 & $\mathrm{~L}$ & Litter \\
\hline-3 to 0 & Of & Leaf structure visible \\
\hline $0-10$ & Ah1 & Black (10YR 2/1), sandy loam, very frequent roots \\
\hline $10-18$ & Ah2 & Dark brown (10YR $3 / 3)$, silty clay loam, frequent roots \\
\hline $18-30$ & AhAl & Dark brown (10YR $3 / 3)$, sandy loam, frequent roots \\
\hline $30-38$ & Al1 & $\begin{array}{l}\text { Clay eluviated (Lessivé), brown (10YR 4/3), sandy loam, gravels, single } \\
\text { vertical roots }\end{array}$ \\
\hline $38-56$ & $\mathrm{Al} 2$ & Brown (10YR 4/3), sandy loam \\
\hline $56-74$ & SwBt & $\begin{array}{l}\text { Clay enriched, dark yellowish brown (10YR } 4 / 4) \text {, mottled, clay loam, } \\
\text { gravels, horizontal roots, iron and manganese concretions, weathered } \\
\text { rock material, mica, single earthworms ( } L \text {. terrestris) }\end{array}$ \\
\hline
\end{tabular}


74-110 (II)SdBt Water impeding, clay enriched, dark yellowish brown (10YR 4/6) and yellowish brown (10YR 5/4), sandy loam, few gravels, old horizontal root channels, clay cutanes clearly visible

110+ IIC Boulder marl

TABLE 4. Timmendorf Forest

\begin{tabular}{|c|c|c|c|c|c|c|}
\hline Lab code & Depth (cm) & $\mathrm{pH}$ & $\mathrm{C}$ & $\delta^{13} \mathrm{C}$ & ${ }^{14} \mathrm{C}$ activity & ${ }^{14} \mathrm{C}$ age \\
\hline HAM-1860 & $0-2$ & 3.5 & 4.77 & -26.8 & $119.51 \pm 0.73$ & Iodern \\
\hline HAM-1861 & $2-4$ & 3.5 & 4.53 & -26.7 & $117.05 \pm 0.73$ & odern \\
\hline HAM-1862 & $4-6$ & 3.5 & 3.37 & -26.6 & $110.35 \pm 0.70$ & Modern \\
\hline HAM-1863 & $6-8$ & 3.4 & 2.76 & -26.5 & $103.61 \pm 0.68$ & Modern \\
\hline HAM-1864 & $8-10$ & 3.5 & 1.47 & -26.5 & $100.21 \pm 0.56$ & Modern \\
\hline HAM-1865 & $10-12$ & 3.6 & 3.21 & -26.4 & $98.72 \pm 0.63$ & $80 \pm 50$ \\
\hline HAM-1866 & $12-14$ & 3.7 & 1.77 & -26.2 & $95.70 \pm 0.62$ & $330 \pm 50$ \\
\hline HAM-1867 & $14-16$ & 3.7 & 1.61 & -26.7 & $93.51 \pm 0.62$ & $510 \pm 50$ \\
\hline HAM-1868 & $16-18$ & 3.7 & 2.61 & -26.3 & $93.98 \pm 0.62$ & $480 \pm 50$ \\
\hline HAM-1869 & $18-20$ & 3.8 & 1.06 & -26.2 & $95.77 \pm 0.61$ & $330 \pm 50$ \\
\hline HAM-1870 & $20-22$ & 3.7 & 0.87 & -25.0 & $94.12 \pm 0.62$ & 50 \\
\hline HAM-1871 & $22-24$ & 3.9 & 0.87 & -26.0 & $92.16 \pm 0.61$ & $640 \pm 50$ \\
\hline HAM-1872 & $24-26$ & 4.0 & 0.68 & -26.2 & $91.92 \pm 0.60$ & $660 \pm 50$ \\
\hline HAM-1873 & $26-28$ & 3.8 & 1.10 & -25.9 & $91.46 \pm 0.61$ & $700 \pm 50$ \\
\hline HAM-1874 & $28-30$ & 3.9 & 0.67 & -25.9 & $91.35 \pm 0.61$ & $710 \pm 50$ \\
\hline HAM-1875 & $30-32$ & 4.0 & 1.04 & -25.9 & $90.11 \pm 0.60$ & $820 \pm 50$ \\
\hline HAM-1876 & $32-34$ & 4.0 & 0.90 & -26.2 & $90.56 \pm 0.61$ & $780 \pm 50$ \\
\hline HAM-1877 & $34-36$ & 4.0 & 1.21 & -26.0 & \pm 0.60 & \pm 50 \\
\hline HAM-1878 & $36-38$ & 4.2 & 0.43 & -25.7 & $91.52 \pm 0.63$ & $700 \pm 60$ \\
\hline HAM-1879 & $38-40$ & 4.0 & 0.69 & -25.6 & $87.70 \pm 0.59$ & $1040 \pm 50$ \\
\hline HAM- 1880 & $40-42$ & 4.1 & 0.24 & -25.5 & $87.35 \pm 0.59$ & \pm 50 \\
\hline HAM-1881 & $42-44$ & 4.1 & 0.39 & -25.6 & $87.01 \pm 0.59$ & $1110 \pm 60$ \\
\hline HAM-1882 & $44-46$ & 4.1 & 0.25 & -25.2 & $85.43 \pm 0.59$ & $1260 \pm 60$ \\
\hline HAM-1883 & $46-48$ & 4.0 & 0.41 & -25.5 & $84.41 \pm 0.61$ & $1350 \pm 60$ \\
\hline HAM-1884 & $48-50$ & 4.2 & 0.13 & -25.3 & $88.72 \pm 0.60$ & $960 \pm 50$ \\
\hline HAM-1885 & $50-52$ & 4.2 & 0.20 & -25.6 & $83.69 \pm 0.58$ & $1420 \pm 60$ \\
\hline HAM-1886 & $52-54$ & 4.2 & 0.10 & -25.2 & $81.48 \pm 0.57$ & $1640 \pm 60$ \\
\hline HAM-1887 & $54-56$ & 4.0 & 0.37 & -25.2 & $88.19 \pm 0.60$ & $1010 \pm 50$ \\
\hline HAM-1888 & $56-58$ & 4.1 & 0.18 & -25.4 & $82.28 \pm 0.57$ & $1560 \pm 60$ \\
\hline HAM-1889 & $58-60$ & 4.2 & 0.13 & -25.3 & $89.60 \pm 0.60$ & $880 \pm 50$ \\
\hline HAM-1890 & $60-62$ & 4.0 & 0.20 & -25.3 & $85.38 \pm 0.59$ & $1260 \pm 60$ \\
\hline HAM-1891 & $62-64$ & 4.1 & 0.39 & -25.5 & $83.13 \pm 0.58$ & $1480 \pm 60$ \\
\hline HAM-1892 & $64-66$ & 3.8 & 0.42 & -24.7 & $79.73 \pm 0.58$ & $1820 \pm 60$ \\
\hline HAM-1893 & $66-68$ & 3.9 & 0.24 & -25.1 & $78.81 \pm 0.58$ & $1910 \pm 60$ \\
\hline HAM-1894 & $68-70$ & 4.0 & 0.26 & -25.1 & $76.41 \pm 0.55$ & $2160 \pm 60$ \\
\hline HAM-1895 & $70-72$ & 4.1 & 0.16 & -25.0 & $75.48 \pm 0.55$ & $2260 \pm 60$ \\
\hline HAM-1896 & $72-74$ & 4.4 & 0.15 & -25.1 & $71.87 \pm 0.53$ & $2650 \pm 60$ \\
\hline HAM-1897 & $74-76$ & 5.0 & 0.15 & -25.1 & $71.20 \pm 0.53$ & $2730 \pm 60$ \\
\hline HAM-1898 & $76-78$ & 5.1 & 0.10 & -24.9 & $75.28 \pm 0.54$ & $2280 \pm 60$ \\
\hline HAM-1899 & $78-80$ & 5.0 & 0.11 & -24.8 & $77.80 \pm 0.56$ & $2020 \pm 60$ \\
\hline HAM-1900 & $80-82$ & 5.0 & 0.18 & -24.9 & $80.02 \pm 0.56$ & $1790 \pm 60$ \\
\hline HAM-1901 & $82-84$ & 5.1 & 0.18 & -24.8 & $83.46 \pm 0.57$ & $1460 \pm 60$ \\
\hline
\end{tabular}




\section{TRITTAU FOREST}

\section{Profile-Related Data}

Location (longitude, latitude):

Location (country, next city or village):

Soil order and type (FAO classification):

Soil order and type (local classification):

Parent material:

Mean annual temperature:

Annual rainfall:

Vegetation and land use:

Site description:

Date of sampling:

Date of measurement:

Collector:

Submitter:

Submitter's comment:

Lab comment:

Literature references: $53^{\circ} 37^{\prime} \mathrm{N}, 10^{\circ} 28^{\prime} \mathrm{E}$

Germany, Trittau (Hahnheide)

Cambic Podzol

Schwach podsolierte Braunerde above Pseudogley-

Parabraunerde

Weichselian boulder cover sand above Weichselian or late Saalean boulder loam (Fuhlsbüttel moraine)

\section{$8.1^{\circ} \mathrm{C}$}

$742 \mathrm{~mm}$

110-yr-old beech stand, forest

$70 \mathrm{~m}$ asl, slope

1982

1983/1984

Becker-Heidmann

Becker-Heidmann

The profile seemed to be disturbed

--

Neue (1980)

Description of the Profile at Trittau Forest:

\begin{tabular}{cll} 
Depth $(\mathrm{cm})$ & Horizon & Description \\
\hline-5 to -3 & L & Litter \\
-3 to -2 & Of & Fungus \\
-2 to 0 & Oh & Many roots \\
$0-4$ & Aeh & Silty loamy sand, gray (10YR 5/1), few roots, undulating border \\
$4-7$ & Bhs & Dark brown (7.5YR 3/2), many fine roots \\
$7-20$ & Bv1 & Yellowish brown (10YR 5/8), many roots \\
$20-32$ & Bv2 & Weathered \\
$32-44$ & SwBv & \\
$44-54$ & IISdBt1 & Loamy sand, brownish yellow (10YR 6/6), very few roots, single vertical \\
& & roots \\
$54+$ & IISdBt2 & Dark brown (10YR 4/6), few roots, manganese concretions
\end{tabular}

TABLE 5. Trittau Forest

\begin{tabular}{ccccccc}
\hline Lab code & Depth $(\mathrm{cm})$ & $\mathrm{pH}$ & $\mathrm{C}$ & $\delta^{13} \mathrm{C}$ & ${ }^{14} \mathrm{C}$ activity & ${ }^{14} \mathrm{C}$ age \\
\hline HAM-1704 & $0-1$ & 3.1 & 4.61 & -27.6 & $96.85 \pm 0.61$ & $215 \pm 50$ \\
HAM-1705 & $1-4$ & 3.1 & 4.05 & -27.4 & $96.78 \pm 0.69$ & $220 \pm 60$ \\
HAM-1708 & $4-7$ & 3.3 & 2.75 & -26.9 & $100.61 \pm 0.61$ & Modern \\
HAM-1709 & $7-10$ & 3.5 & 0.99 & -26.7 & $99.86 \pm 0.61$ & Modern \\
HAM-1710 & $10-12$ & 3.7 & 1.02 & -26.8 & $98.81 \pm 0.61$ & $65 \pm 50$ \\
HAM-1711 & $12-14$ & 3.9 & 1.11 & -26.6 & $98.58 \pm 0.61$ & $90 \pm 50$ \\
HAM-1712 & $14-16$ & 4.1 & 0.72 & -26.5 & $97.82 \pm 0.61$ & $155 \pm 50$ \\
HAM-1713 & $16-18$ & 4.0 & 1.08 & -26.5 & $94.50 \pm 0.60$ & $430 \pm 50$ \\
HAM-1714 & $18-20$ & 4.1 & 1.02 & -26.4 & $96.36 \pm 0.61$ & $275 \pm 50$ \\
HAM-1715 & $20-22$ & 4.1 & 0.76 & -26.4 & $94.85 \pm 0.60$ & $400 \pm 50$
\end{tabular}


TABLE 5. Trittau Forest (Continued)

\begin{tabular}{cccccrc}
\hline Lab code & Depth $(\mathrm{cm})$ & $\mathrm{pH}$ & $\mathrm{C}$ & $\delta^{13} \mathrm{C}$ & \multicolumn{1}{c}{${ }^{14} \mathrm{C}$ activity } & ${ }^{14} \mathrm{C}$ age \\
\hline HAM-1716 & $22-24$ & 4.1 & 0.77 & -26.4 & $96.64 \pm 0.60$ & $255 \pm 50$ \\
HAM-1717 & $24-26$ & 4.1 & 0.66 & -26.3 & $93.69 \pm 0.60$ & $500 \pm 50$ \\
HAM-1718 & $26-28$ & 4.1 & 0.54 & -26.2 & $95.26 \pm 0.60$ & $370 \pm 50$ \\
HAM-1719 & $28-30$ & 4.1 & 0.44 & -26.0 & $97.95 \pm 0.61$ & $150 \pm 50$ \\
HAM-1720 & $30-32$ & 4.1 & 0.38 & -26.0 & $95.66 \pm 0.60$ & $340 \pm 50$ \\
HAM-1721 & $32-34$ & 4.1 & 0.30 & -25.6 & $105.25 \pm 0.63$ & Modern \\
HAM-1722 & $34-36$ & 4.1 & 0.21 & -25.3 & $97.56 \pm 0.85$ & $190 \pm 70$ \\
HAM-1723 & $36-38$ & 4.0 & 0.17 & -25.0 & $97.24 \pm 0.61$ & $225 \pm 50$ \\
HAM-1724 & $38-40$ & 4.0 & 0.15 & -25.1 & $94.07 \pm 0.59$ & $490 \pm 50$ \\
HAM-1725 & $40-42$ & 4.0 & 0.13 & -24.6 & $94.65 \pm 0.60$ & $450 \pm 50$ \\
HAM-1726 & $42-44$ & 3.9 & 0.12 & -25.0 & $122.70 \pm 0.69$ & Modern \\
HAM-1727 & $44-46$ & 3.9 & 0.10 & -24.9 & $101.57 \pm 0.62$ & Modern \\
HAM-1728 & $46-48$ & 3.8 & 0.10 & -24.8 & $102.90 \pm 0.63$ & Modern \\
HAM-1729 & $48-50$ & 3.8 & 0.08 & -24.2 & $93.99 \pm 0.69$ & $510 \pm 60$ \\
HAM-1730 & $50-52$ & 3.7 & 0.08 & -24.3 & $98.96 \pm 0.61$ & $95 \pm 50$ \\
HAM-1731 & $52-54$ & 3.7 & 0.07 & -24.3 & $123.23 \pm 1.38$ & Modern \\
HAM-1732 & $54-56$ & 3.7 & 0.08 & -24.3 & $117.03 \pm 2.02$ & Modern \\
HAM-1733 & $56-58$ & 3.7 & 0.08 & -24.4 & $115.97 \pm 1.37$ & Modern \\
HAM-1734 & $58-60$ & 3.7 & 0.07 & -24.2 & $124.35 \pm 1.93$ & Modern \\
\hline
\end{tabular}

Bulk density (Neue 1980: Table 24, p. 185)

\begin{tabular}{cc}
\hline $\begin{array}{c}\text { Depth } \\
(\mathrm{cm})\end{array}$ & $\begin{array}{c}\text { Bulk density } \\
\left(\mathrm{g} \mathrm{cm}^{-3}\right)\end{array}$ \\
\hline $0-3$ & 1.13 \\
$3-5$ & 1.29 \\
$5-9$ & 1.30 \\
$9-20$ & 1.34 \\
$20-38$ & 1.67 \\
$38-48$ & 1.72 \\
$48-68$ & 1.81 \\
\hline
\end{tabular}

\section{KLEIN ALTENDORF}

\section{Profile-Related Data}

Location (longitude, latitude):

Location (country, next city or village):

Soil order and type (FAO classification):

Soil order and type (local classification):

Parent material:

Mean annual temperature:

Annual rainfall:

Vegetation and land use:

Site description:

Date of sampling:

Date of measurement:

Collector: $50^{\circ} 38^{\prime} \mathrm{N}, 6^{\circ} 59^{\prime} \mathrm{E}$

Germany, Klein Altendorf south of Bonn

Haplic Luvisol

Parabraunerde

Würmian loess

$8.9^{\circ} \mathrm{C}$

$625 \mathrm{~mm}$

Test area for fruit production, 1956-1981 apples, 1981-1982 cherries

$177.5 \mathrm{~m}$ asl, plane

1982

1984

Becker-Heidmann 
Submitter:

Submitter's comment:

Lab comment:

Literature references:

\author{
Becker-Heidmann \\ See literature references \\ Becker-Heidmann (1989); Scharpenseel and Becker- \\ Heidmann (1994b)
}

\section{Description of the Profile at Klein Altendorf Experimental Station}

\begin{tabular}{lll} 
Depth $(\mathrm{cm})$ & Horizon & Description \\
\hline $0-14$ & Ap1 & Light yellowish brown (10YR 6/4), loamy silt, frequent roots \\
$14-24$ & Ap2 & Very frequent fine roots, loamy silt \\
$24-40$ & ApAl & Clay eluviated, light yellowish brown (10YR 6/4), loamy silt, few roots \\
$40-54$ & Al1 & Clay eluviated, very pale brown (10YR 7/4), loamy silt, very few roots \\
$54-68$ & Al2 & Very pale brown (10YR 7/4), loamy silt \\
$68-78$ & Bt1 & Clay enriched, brownish yellow (10YR 6/6), loamy silt, no roots \\
$78-92+$ & Bt2 & Bright yellowish brown (10YR 6/6), loamy silt
\end{tabular}

TABLE 6. Klein Altendorf

\begin{tabular}{ccccccr}
\hline Lab code & Depth $(\mathrm{cm})$ & pH & C & \multicolumn{1}{c}{${ }^{13} \mathrm{C}$} & \multicolumn{1}{c}{14 activity } & \multicolumn{1}{c}{${ }^{14}$ age } \\
\hline HAM-1810 & $0-2$ & 6.5 & 0.70 & -25.0 & $87.25 \pm 0.66$ & $1090 \pm 60$ \\
HAM-1811 & $2-4$ & 6.6 & 0.82 & -25.2 & $86.61 \pm 0.66$ & $1150 \pm 60$ \\
HAM-1812 & $4-6$ & 6.4 & 0.70 & -25.0 & $89.04 \pm 0.67$ & $930 \pm 60$ \\
HAM-1813 & $6-8$ & 6.2 & 0.87 & -25.7 & $85.77 \pm 0.66$ & $1220 \pm 60$ \\
HAM-1814 & $8-10$ & 6.0 & 0.88 & -24.9 & $88.40 \pm 0.66$ & $990 \pm 60$ \\
HAM-1815 & $10-12$ & 5.9 & 0.97 & -25.0 & $88.72 \pm 0.66$ & $960 \pm 60$ \\
HAM-1816 & $12-14$ & 5.8 & 1.00 & -25.9 & $89.02 \pm 0.84$ & $920 \pm 80$ \\
HAM-1817 & $14-16$ & 5.8 & 1.11 & -25.1 & $94.35 \pm 0.69$ & $470 \pm 60$ \\
HAM-1818 & $16-18$ & 6.0 & 1.01 & -24.9 & $87.49 \pm 0.66$ & $1080 \pm 60$ \\
HAM-1819 & $18-20$ & 6.2 & 1.19 & -25.7 & $95.23 \pm 0.62$ & $380 \pm 50$ \\
HAM-1820 & $20-22$ & 6.1 & 1.16 & -25.7 & $96.43 \pm 0.67$ & $280 \pm 60$ \\
HAM-1821 & $22-24$ & 6.0 & 1.17 & -26.0 & $96.30 \pm 0.67$ & $290 \pm 60$ \\
HAM-1822 & $24-26$ & 6.0 & 0.92 & -24.7 & $89.99 \pm 0.65$ & $850 \pm 60$ \\
HAM-1823 & $26-28$ & 6.1 & 0.81 & -25.4 & $89.09 \pm 0.63$ & $920 \pm 60$ \\
HAM-1824 & $28-30$ & 6.2 & 0.74 & -24.5 & $90.43 \pm 0.65$ & $820 \pm 60$ \\
HAM-1825 & $30-32$ & 6.3 & 0.58 & -23.9 & $89.50 \pm 0.64$ & $910 \pm 60$ \\
HAM-1826 & $32-34$ & 6.3 & 0.44 & -24.2 & $87.90 \pm 0.62$ & $1050 \pm 60$ \\
HAM-1827 & $34-36$ & 6.4 & 0.43 & -24.1 & $89.98 \pm 0.65$ & $860 \pm 60$ \\
HAM-1828 & $36-38$ & 6.3 & 0.41 & -24.1 & $87.49 \pm 0.64$ & $1090 \pm 60$ \\
HAM-1829 & $38-40$ & 6.1 & 0.38 & -24.0 & $87.17 \pm 0.63$ & $1120 \pm 60$ \\
HAM-1830 & $40-42$ & 6.1 & 0.37 & -24.6 & $84.50 \pm 0.62$ & $1360 \pm 60$ \\
HAM-1831 & $42-44$ & 6.3 & 0.38 & -24.7 & $81.79 \pm 0.62$ & $1620 \pm 60$ \\
HAM-1832 & $44-46$ & 6.2 & 0.37 & -23.5 & $79.80 \pm 0.63$ & $1840 \pm 60$ \\
HAM-1833 & $46-48$ & 6.3 & 0.36 & -23.3 & $81.17 \pm 0.61$ & $1700 \pm 60$ \\
HAM-1834 & $48-50$ & 6.4 & 0.36 & -24.3 & $80.51 \pm 0.61$ & $1750 \pm 60$ \\
HAM-1835 & $50-52$ & 6.5 & 0.36 & -24.9 & $79.67 \pm 0.61$ & $1830 \pm 60$ \\
HAM-1836 & $52-54$ & 6.2 & 0.35 & -23.8 & $78.80 \pm 0.60$ & $1930 \pm 60$ \\
HAM-1837 & $54-56$ & 6.2 & 0.36 & -24.0 & $79.38 \pm 0.75$ & $1870 \pm 80$ \\
HAM-1838 & $56-58$ & 6.5 & 0.36 & -23.8 & $74.43 \pm 0.59$ & $2390 \pm 60$ \\
HAM-1839 & $58-60$ & 6.5 & 0.36 & -23.9 & $72.15 \pm 0.57$ & $2640 \pm 60$ \\
HAM-1840 & $60-62$ & 6.5 & 0.39 & -24.1 & $68.25 \pm 0.56$ & $3080 \pm 70$
\end{tabular}


TABLE 6. Klein Altendorf (Continued)

\begin{tabular}{ccccccc}
\hline Lab code & Depth $(\mathrm{cm})$ & $\mathrm{pH}$ & $\mathrm{C}$ & $\delta^{13} \mathrm{C}$ & ${ }^{14} \mathrm{C}$ activity & ${ }^{14} \mathrm{C}$ age \\
\hline HAM-1841 & $62-64$ & 6.6 & 0.35 & -23.9 & $67.36 \pm 0.55$ & $3190 \pm 70$ \\
HAM-1842 & $64-66$ & 6.3 & 0.35 & -23.6 & $66.26 \pm 0.55$ & $3330 \pm 70$ \\
HAM-1843 & $66-68$ & 6.3 & 0.34 & -23.8 & $65.00 \pm 0.54$ & $3480 \pm 70$ \\
HAM-1844 & $68-70$ & 6.6 & 0.38 & -23.8 & $60.60 \pm 0.53$ & $4040 \pm 70$ \\
HAM-1845 & $70-72$ & 6.6 & 0.38 & -24.2 & $59.93 \pm 0.53$ & $4130 \pm 70$ \\
HAM-1846 & $72-74$ & 6.4 & 0.39 & -24.9 & $58.72 \pm 0.53$ & $4280 \pm 70$ \\
HAM-1847 & $74-76$ & 6.5 & 0.38 & -24.9 & $59.30 \pm 0.52$ & $4200 \pm 70$ \\
HAM-1848 & $76-78$ & 6.6 & 0.39 & -24.6 & $62.88 \pm 0.56$ & $3730 \pm 70$ \\
HAM-1849 & $78-80$ & 6.3 & 0.33 & -24.5 & $64.05 \pm 0.54$ & $3590 \pm 70$ \\
HAM-1850 & $80-82$ & 6.4 & 0.31 & -24.8 & $63.50 \pm 0.54$ & $3650 \pm 70$ \\
HAM-1851 & $82-84$ & 6.4 & 0.26 & -24.6 & $60.48 \pm 0.58$ & $4050 \pm 80$ \\
HAM-1852 & $84-86$ & 6.6 & 0.44 & -24.6 & $60.40 \pm 0.53$ & $4060 \pm 70$ \\
HAM-1853 & $86-88$ & 6.7 & 0.44 & -24.6 & $60.55 \pm 0.52$ & $4040 \pm 70$ \\
HAM-1854 & $88-90$ & 6.6 & 0.35 & -24.6 & $59.60 \pm 0.52$ & $4160 \pm 70$ \\
HAM-1855 & $90-92$ & 6.5 & 0.35 & -24.7 & $62.33 \pm 0.53$ & $3800 \pm 70$ \\
\hline
\end{tabular}

\section{SAvarit, Profile 1}

\section{Profile-Related Data}

Location (longitude, latitude):

$46^{\circ} 07^{\prime} \mathrm{N}, 0^{\circ} 50^{\prime} \mathrm{W}$

Location (country, next city or village):

France, Savarit, $60 \mathrm{~km}$ southwest of Niort in PoitouCharentes province

Soil order and type (FAO classification): $\quad$ Rendzic Leptosol

Soil order and type (USDA classification): Lithic Rendoll, clayey skeletal mesic udic

Soil order and type (local classification):

Parent material:

Groies

Mean annual temperature:

Malm

Annual rainfall:

Vegetation and land use:

$15^{\circ} \mathrm{C}$

$790 \mathrm{~mm}$

Wheat, sunflower, currently experimental site for studies on biological nitrogen fixation (BNF)

Site description:

Date of sampling: $24 \mathrm{~m}$ asl, slight slope, top of a toposequence

Date of measurement: 1990

1990

Collector:

Becker-Heidmann

Submitter:

Becker-Heidmann

Submitter's comment:

Both Savarit soil profiles contain large amounts of carbonate. Therefore, after measuring the $\mathrm{pH}$ of the total samples, carbon, $\delta^{13} \mathrm{C}$ and ${ }^{14} \mathrm{C}$ activity were measured on the inorganic and organic fraction of the samples separately. The high carbon content, ca. 3-4\% throughout the profile, supports good crop production. The amounts of organic and inorganic carbon are nearly equal except in the uppermost $2 \mathrm{~cm}$. The $\delta^{13} \mathrm{C}$ values of organic and inorganic carbon are very distinguishable, with ca. $-22 \%$ for organic and $-2 \%$ for inorganic C. We detected no substantial exchange between carbonate- and Humus-C. The humus is well decomposed throughout the whole profile, consistently with high $\mathrm{pH}$. The ${ }^{14} \mathrm{C}$ activity of the organic carbon is rather low $(<100 \mathrm{pmC})$, which reflects good decomposition of the humus. The ${ }^{14} \mathrm{C}$ activity of the carbonate is low, corresponding to a high ${ }^{14} \mathrm{C}$ age between 8000 and $11,000 \mathrm{BP}$. The reason for its great variation over the soil profile is not clear.

Literature references:

Becker-Heidmann (1992); Drachenberg (1992) 


\section{Description of the Profile at Savarit 1}

\begin{tabular}{cll} 
Depth $(\mathrm{cm})$ & Horizon & Description \\
\hline $0-14$ & Ap & Silty loam, dark brown, 10 YR 3/3, calcareous, gravels \\
$14-20$ & $\mathrm{R}$ & Clayey silt, brown 10 YR 4/3, calcareous, gravels \\
$20+$ & $\mathrm{C}$ &
\end{tabular}

TABLE 7. Savarit, Profile 1

\begin{tabular}{lcccccccccc}
\hline Lab code & $\begin{array}{c}\text { Depth } \\
(\mathrm{cm})\end{array}$ & $\mathrm{pH}$ & $\mathrm{C}_{\text {org }}$ & $\delta^{13} \mathrm{C}_{\text {org }}$ & $\begin{array}{c}{ }^{14} \mathrm{C}_{\text {org }} \\
\text { activity }\end{array}$ & ${ }^{14} \mathrm{C}_{\text {org }}$ age & $\mathrm{C}_{\text {in }}$ & $\delta^{13} \mathrm{C}_{\text {in }}$ & $\begin{array}{c}{ }^{14} \mathrm{C}_{\text {in }} \\
\text { activity }\end{array}$ & ${ }^{14} \mathrm{C}_{\text {in }}$ age \\
\hline HAM-3035 & $0-2$ & 7.4 & 2.83 & -22.3 & $96.10 \pm 0.56$ & $365 \pm 50$ & 3.81 & -2.1 & $39.96 \pm 0.40$ & $7370 \pm 80$ \\
HAM-3036 & $2-4$ & 7.5 & 3.37 & -20.8 & $95.35 \pm 0.57$ & $450 \pm 50$ & 3.52 & -2.3 & $28.53 \pm 0.36$ & $10,080 \pm 110$ \\
HAM-3037 & $4-6$ & 7.4 & 3.22 & -21.0 & $95.46 \pm 0.56$ & $435 \pm 50$ & 3.67 & -2.4 & $23.21 \pm 0.35$ & $11,730 \pm 130$ \\
HAM-3038 & $6-8$ & 7.4 & 3.54 & -22.2 & $95.02 \pm 0.58$ & $460 \pm 50$ & 3.40 & -2.8 & $35.95 \pm 0.38$ & $8220 \pm 90$ \\
HAM-3039 & $8-10$ & 7.4 & 3.48 & -21.6 & $95.06 \pm 0.56$ & $460 \pm 50$ & 3.26 & -2.5 & $33.66 \pm 0.52$ & $8750 \pm 130$ \\
HAM-3040 & $10-12$ & 7.3 & 3.37 & -21.7 & $95.58 \pm 0.56$ & $415 \pm 50$ & 3.45 & -2.7 & $31.64 \pm 0.37$ & $9240 \pm 100$ \\
HAM-3041 & $12-14$ & 7.4 & 3.19 & -21.0 & $95.39 \pm 0.56$ & $445 \pm 50$ & 3.64 & -2.5 & $31.52 \pm 0.37$ & $9270 \pm 100$ \\
HAM-3042 & $14-16$ & 7.4 & 3.38 & -20.1 & $93.59 \pm 0.55$ & $610 \pm 50$ & 3.59 & -2.7 & $25.13 \pm 0.35$ & $11,090 \pm 120$ \\
HAM-3043 & $16-18$ & 7.4 & 2.99 & -21.5 & $91.65 \pm 0.55$ & $760 \pm 50$ & 3.57 & -2.3 & $24.67 \pm 0.35$ & $11,240 \pm 120$ \\
\hline
\end{tabular}

\section{SAVARIT, Profile 2}

\section{Profile-Related Data}

Same as Profile 1, except:

Site description: Bottom of the toposequence

Submitter's comment:

The values of $\mathrm{pH}$, organic carbon, $\delta^{13} \mathrm{C}$ and ${ }^{14} \mathrm{C}$ are similar to those of Profile 1 . The carbonate content is higher, and even more striking; the variation of the values is relatively high. The $\delta^{13} \mathrm{C}$ value is generally $c a$. $2 \%$ higher. The $\delta^{13} \mathrm{C}$ depth distribution of the organic carbon shows an unexpected rise from $-20 \%$ to $-16 \%$ at $c a .8 \mathrm{~cm}$. The ${ }^{14} \mathrm{C}$ activity of the carbonate fraction changes several times between 20 and $30 \mathrm{pMC}$; the corresponding ${ }^{14} \mathrm{C}$ age differs between 10,000 and $15,000 \mathrm{BP}$ within the lower half of the soil profile. The reason for this irregularity is not clear.

\section{Description of Profile at Savarit 2}

\begin{tabular}{cll} 
Depth $(\mathrm{cm})$ & Horizon & Description \\
\hline $0-18$ & Ap & Silty loam, brown 10 YR 4/3, calcareous, many fine roots, gravels \\
$18-30$ & R & Silty loam, brown 10 YR 4/3, calcareous, some fine roots, gravels \\
$30+$ & C &
\end{tabular}

TABLE 8. Savarit, Profile 2

\begin{tabular}{ccccccccccc}
\hline Lab code & $\begin{array}{c}\text { Depth } \\
(\mathrm{cm})\end{array}$ & pH & $\mathrm{C}_{\text {org }}$ & $\delta^{13} \mathrm{C}_{\text {org }}$ & $\begin{array}{c}{ }^{14} \mathrm{C}_{\text {org }} \\
\text { activity }\end{array}$ & ${ }^{14} \mathrm{C}_{\text {org }}$ age & $\mathrm{C}_{\text {in }}$ & $\delta^{13} \mathrm{C}_{\text {in }}$ & ${ }^{14} \mathrm{C}_{\text {in }}$ \\
activity & ${ }^{14} \mathrm{C}_{\text {in }}$ age \\
\hline HAM-3044 & $0-2$ & 7.4 & 3.00 & -20.6 & $95.32 \pm 0.57$ & $455 \pm 50$ & 5.28 & -1.9 & $16.83 \pm 0.33$ & $14,310 \pm 170$ \\
HAM-3045 & $2-4$ & 7.5 & 3.18 & -19.5 & $94.24 \pm 0.56$ & $565 \pm 50$ & 5.09 & -1.4 & $16.10 \pm 0.33$ & $14,670 \pm 170$ \\
HAM-3046 & $4-6$ & 7.4 & 3.36 & -20.1 & $95.87 \pm 0.56$ & $420 \pm 50$ & 5.00 & -1.5 & $20.11 \pm 0.34$ & $12,880 \pm 150$ \\
HAM-3047 & $6-8$ & 7.4 & 3.24 & -19.8 & $96.71 \pm 0.56$ & $355 \pm 50$ & 5.22 & -1.7 & $21.50 \pm 0.34$ & $12,350 \pm 140$ \\
HAM-3048 & $8-10$ & 7.5 & 3.33 & -16.6 & $97.90 \pm 0.57$ & $305 \pm 50$ & 5.15 & -1.1 & $26.31 \pm 0.35$ & $10,730 \pm 120$ \\
HAM-3049 & $10-12$ & 7.3 & 3.23 & -19.6 & $93.15 \pm 0.56$ & $660 \pm 50$ & 5.02 & -1.6 & $18.28 \pm 0.33$ & $13,650 \pm 160$ \\
HAM-3050 & $12-14$ & 7.3 & 3.41 & -21.4 & $95.39 \pm 0.56$ & $440 \pm 50$ & 4.93 & -1.5 & $26.77 \pm 0.36$ & $10,590 \pm 120$ \\
HAM-3051 & $14-16$ & 7.4 & 3.48 & -20.3 & $92.57 \pm 0.56$ & $700 \pm 50$ & 5.10 & -1.6 & $15.66 \pm 0.32$ & $14,890 \pm 180$
\end{tabular}


TABLE 8. Savarit, Profile 2 (Continued)

\begin{tabular}{|c|c|c|c|c|c|c|c|c|c|c|}
\hline Lab code & $\begin{array}{c}\text { Depth } \\
(\mathrm{cm})\end{array}$ & $\mathrm{pH}$ & $\mathrm{C}_{\text {org }}$ & $\delta^{13} C_{\text {org }}$ & $\begin{array}{c}{ }^{14} \mathrm{C}_{\text {org }} \\
\text { activity }\end{array}$ & ${ }^{14} \mathrm{C}_{\text {org }}$ age & $\mathrm{C}_{\mathrm{in}}$ & $\delta^{13} C_{i n}$ & $\begin{array}{c}{ }^{14} \mathrm{C}_{\text {in }} \\
\text { activity }\end{array}$ & ${ }^{14} \mathrm{C}_{\text {in }}$ age \\
\hline HAM-3052 & $16-18$ & 7.4 & 2.99 & -20.6 & $97.58 \pm 0.56$ & $265 \pm 50$ & 5.54 & -1.1 & $28.61 \pm 0.36$ & $10,050 \pm 110$ \\
\hline HAM-3053 & $18-20$ & 7.5 & 2.36 & -19.9 & $95.23 \pm 0.56$ & $475 \pm 50$ & 5.57 & -0.8 & $19.29 \pm 0.34$ & $13,220 \pm 150$ \\
\hline HAM-3054 & $20-22$ & 7.5 & 2.21 & -17.7 & $93.45 \pm 0.55$ & $660 \pm 50$ & 5.61 & -0.5 & $22.86 \pm 0.34$ & $11,850 \pm 130$ \\
\hline HAM-3055 & $22-24$ & 7.5 & 1.94 & -19.4 & $92.59 \pm 0.55$ & $710 \pm 50$ & 5.36 & -0.7 & $20.84 \pm 0.34$ & $12,600 \pm 140$ \\
\hline
\end{tabular}

\section{AKKo}

\section{Profile-Related Data}

Location (longitude, latitude):

$32^{\circ} 54^{\prime} \mathrm{N}, 35^{\circ} 15^{\prime} \mathrm{E}$

Location (country, next city or village): $\quad$ Israel, Akko

Soil order and type (USDA classification): Typic Pelloxerert, fine, mixed (calcareous), thermic

Soil order and type (local classification): Grumusol

Mean annual temperature:

$19^{\circ} \mathrm{C}$

Annual rainfall:

$626 \mathrm{~mm}$

Vegetation and land use: $\quad$ Noncultivated

Site description:

The profile was located on site GR-4 of the Western Galilee Experimental Farm in the coastal plain near Akko, in the north of Israel. The soil had been studied before by Yaalon and Kalmar $(1972,1978)$ regarding its pedoturbation by cracking and swelling processes.

Date of sampling:

Date of measurement:

1985

Collector:

$1986 / 1987$

Submitter:

Dov Kalmar, Dan Yaalon

Lab comment:

Dov Kalmar

This Vertisol has a pH close to 8 throughout the profile. Accordingly, the carbonate content is on average as high as the organic carbon content, almost exceeding the latter in the bottom of the soil, and only less in the A1 horizon. The $\delta^{13} \mathrm{C}$ value of the organic matter corresponds to the $\mathrm{C}_{3}$-type crops grown here, with $-28 \% 0$ at the soil surface, and also the shift with depth of $c a$. 3\%o is in the expected range for terrestrial soils (cf. Becker-Heidmann 1989; Bertram 1986). The $\delta^{13} \mathrm{C}$ value of the inorganic carbon ca. $-13 \%$ is low compared to primary carbonate with $0 \%$. As in the other carbonate-containing profiles, an exchange between organic and inorganic matter has occurred within the whole profile. The shift, when related to the absolute $\delta^{13} \mathrm{C}$ values of the organic fraction, is comparable. Therefore, the same mechanism can be assumed. The exchange is also obvious when comparing the ${ }^{14} \mathrm{C}$ activity values. The carbonate of this soil is assumed to belong to the original sediment from which the soil developed, i.e., it should have a very low ${ }^{14} \mathrm{C}$ activity corresponding to a high ${ }^{14} \mathrm{C}$ age. Instead, it follows a depth curve similar to that of the ${ }^{14} \mathrm{C}$ activity of the organic carbon, at a lower level, ending up with a ${ }^{14} \mathrm{C}$ activity of $c a .20 \mathrm{pMC}$, corresponding to a ${ }^{14} \mathrm{C}$ age of $14,000 \mathrm{BP}$. The ${ }^{14} \mathrm{C}$ activities of the organic fraction as well as of the carbonate carbon fraction decrease distinctively with depth, and there is no discontinuity in the depth curve. So far, a pedoturbation, as expected for a Vertisol, could not be found.

Literature references:

Becker-Heidmann (1990); Yaalon and Kalmar (1972)

\section{Description of the Profile at Akko}

\begin{tabular}{cll} 
Depth $(\mathrm{cm})$ & Horizon & Description \\
\hline $0-15$ & A & $\begin{array}{c}\text { Heavy clay, very dark grayish brown, very small carbonate nodules, } \\
\text { many fine and medium roots }\end{array}$ \\
15-40 & B1 & $\begin{array}{c}\text { Heavy clay, very dark grayish brown, very small carbonate nodules, } \\
\text { many fine roots }\end{array}$
\end{tabular}




$\begin{array}{ccc}40-100 & \text { B21 } & \begin{array}{c}\text { Heavy clay, very dark grayish brown, very small carbonate nodules, } \\ \text { many slickensides, very few fine roots } \\ \text { Heavy clay, very dark grayish brown, very small carbonate nodules, } \\ \text { many well-expressed slickensides, very few fine roots }\end{array} \\ 100-140 & \text { B22 } & \begin{array}{c}\text { Heavy clay, very dark grayish brown, some carbonate nodules, many } \\ \text { slickensides, no roots }\end{array}\end{array}$

TABLE 9. Akko

\begin{tabular}{|c|c|c|c|c|c|c|c|c|c|c|}
\hline $\mathrm{L} a$ & $\begin{array}{l}\text { Depth } \\
\text { (cm) }\end{array}$ & $\mathrm{pH}$ & 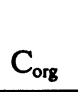 & $C_{\text {org }}$ & $\mathrm{xg}$ act. & Corg age & $\mathrm{C}_{\text {in }}$ & 0 & ${ }^{14} \mathrm{C}_{\text {in }}$ act. & in a \\
\hline 1 & $0-2$ & 7.4 & 4 & 26.5 & $1.58 \pm 0.87$ & Modern & 0.17 & -15.5 & $3.68 \pm 0.70$ & $70 \pm 60$ \\
\hline & & & & & & & & & & \\
\hline & 4 & 7.7 & 1.13 & -26.1 & & & 0.04 & -12.2 & $69.73 \pm 0.77$ & $900 \pm 100$ \\
\hline & & 7.6 & 0.96 & -24.7 & $106.44 \pm$ & & 0.17 & -15.6 & & \\
\hline & & 7.5 & & & & & & -13.5 & & \\
\hline & 10 & 7.7 & & & & & 0.32 & -12.4 & & \\
\hline & & 7.7 & 0.67 & & & & & -13.0 & & \\
\hline & & 7.6 & 0.78 & -24.7 & & & 0.20 & & 61 & \\
\hline & & 7.6 & 0.71 & -2 & & & & & & \\
\hline & $21-2$ & 7.8 & 0.59 & & & & & -12.8 & & \\
\hline & & 7.7 & & -24.2 & & & & -13.2 & & \\
\hline & & 7.7 & & & & & & .7 & & \\
\hline & & 8.0 & 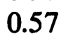 & & & & & & & \\
\hline & & 9 & & & & & & & & \\
\hline & & 7.8 & & & & & & .5 & & \\
\hline & & 7.8 & 1 & & & & & & & \\
\hline & & 7.8 & & & & & & & & \\
\hline & & 7.9 & 0. & & & & & & & \\
\hline & & 7.9 & 55 & -2 & & & & & & \\
\hline & & 7.9 & & & & & & & & \\
\hline & & 7.9 & 0.40 & & & & & & & \\
\hline & & 9 & & & & & & & & \\
\hline & & 7.5 & 0. & & & & & & & 70 \\
\hline & & 7.9 & & & & & 4 & -14.1 & $\pi$ & \\
\hline & & 1 & & & & & & & & \\
\hline & & 0 & & & & & & & & \\
\hline & & 9 & & & & & & & & \\
\hline & & 7.9 & & & & & & & & \\
\hline & & 8.0 & & & & & & & & \\
\hline & & 8.0 & & & & & & & & \\
\hline & & 8.0 & & & & & & & & \\
\hline & & 8.0 & & & & & & & & \\
\hline & & 3 & & & & & & & & \\
\hline & & 7.8 & & & & & & & & \\
\hline & & & & & & & & & & \\
\hline & & 7.7 & & & & & & & & \\
\hline & & 8.2 & & & & & & & & \\
\hline & & & & & & & & & & \\
\hline & & 8.2 & & & & & & & & \\
\hline & & 7.9 & & & & & & & & \\
\hline & & 8.0 & & & & & & & & \\
\hline & & 8.1 & & & & & & & & \\
\hline & & 8.2 & & & & & & & & \\
\hline & & \&? & & & & & & & & \\
\hline & & & & & & & & & & \\
\hline & & 8.2 & & & & & & & & \\
\hline & & & & & & & & & & \\
\hline
\end{tabular}


Bulk density (Yaalon and Kalmar 1972)

\begin{tabular}{cc}
\hline Depth $(\mathrm{cm})$ & Bulk density $\left(\mathrm{g} \mathrm{cm}^{-3}\right)$ \\
\hline $0-15$ & 1.25 \\
$15-100$ & 1.31 \\
$100-140$ & 1.36 \\
$140-185$ & 1.37 \\
\hline
\end{tabular}

\section{QEDMA}

\section{Profile-Related Data}

Location (longitude, latitude):

Location (country, next city or village):

Soil order and type (FAO classification):

Soil order and type (USDA classification):

Soil order and type (local classification):

Parent material:

Mean annual temperature:

Annual rainfall:

Vegetation and land use:

Site description:

The profile was located at a cement mining pit. Therefore, the soil was sampled down to $9 \mathrm{~m}$, giving the chance to study the differences of soil and sediment.

Date of sampling:

Date of measurement:

Collectors:

Submitter:

Submitter's comment:

The $\mathrm{pH}$ is nearly the same in value and depth curve as in the Akko profile. The organic carbon content is generally extremely low $(<0.5 \%)$, whereas the carbonate carbon content is comparably high, $2.5 \%$ in the topsoil and between 0.5 and $1 \%$ in layer $\mathrm{C}$. The lowest sediment layer, Cca, again has $1.5 \%$. Although the $\delta^{13} \mathrm{C}$ of the inorganic carbon is nearly homogeneous through the profile, the $\delta^{13} \mathrm{C}$ value of the organic carbon shows a great variability and an increasing tendency down to $140 \mathrm{~cm}$. It can be concluded that decomposition processes, increasing the $\delta^{13} \mathrm{C}$ value of the soil remaining residue fraction, are considerable, but that pedoturbation has disturbed the expected linearily increasing depth curve. The inorganic carbon is obviously not influenced by the actual $\delta^{13} \mathrm{C}$ of the organic matter. The constant $\delta^{13} \mathrm{C}$ value of $-10 \%$ throughout the profile indicates that the exchange process between organic and inorganic carbon occurred a long time ago. This interpretation is supported by the ${ }^{14} \mathrm{C}$ activity of the inorganic carbon, which is very low, $c a .40 \%$, corresponding to a ${ }^{14} \mathrm{C}$ age of nearly $8000 \mathrm{BP}$. Only the topmost surface layers seem to take part in a recent exchange process. The ${ }^{14} \mathrm{C}$ activity depth distribution curves of both organic and inorganic carbon show only a very slight decrease down to a depth of $90 \mathrm{~cm}$, which is the maximum depth of observed cracks, and decrease more steeply in the lower part of the profile. In this soil, in contrast to the Akko soil, a distinctive self-mulching effect can therefore be recognized from the ${ }^{14} \mathrm{C}$ activity curves. The increase in $\delta^{13} \mathrm{C}$ can be explained by the comparison of transport and mulching speeds: Peloturbation lasts $2000 \mathrm{yr}$ and is slower than organic carbon transport (Yaalon, personal communication).

Literature references:

Becker-Heidmann (1990)

\section{Description of the Profile at Qedma:}

\begin{tabular}{cll} 
Depth $(\mathrm{cm})$ & Horizon & Description \\
\hline $0-8$ & A11 & Heavy clay, very dark grayish brown \\
8-26 & A12 & Heavy clay, very dark grayish brown
\end{tabular}




\begin{tabular}{|c|c|c|}
\hline $26-56$ & B1 & Dry and heavy clay, carbonate nodules at $52-56 \mathrm{~cm}$ \\
\hline $56-112$ & B21 & $\begin{array}{l}\text { Heavy clay, very dark grayish brown many well expressed slickensides, } \\
\text { polyeder, Mn concretions, cracks down to } 100-112 \mathrm{~cm}\end{array}$ \\
\hline $112-140$ & B22 & Heavy clay, very dark grayish brown, many slickensides \\
\hline $140-250$ & B23 & Slickensides, carbonate nodules at $250 \mathrm{~cm}$ \\
\hline $250-750$ & C & Bedrock \\
\hline $750-900$ & $\mathrm{Cca}$ & Calcic horizon, bedrock \\
\hline
\end{tabular}

TABLE 10. Qedma

\begin{tabular}{|c|c|c|c|c|c|c|c|c|c|c|}
\hline Lab code & $\begin{array}{l}\text { Depth } \\
\text { (cm) }\end{array}$ & $\mathrm{pH}$ & $\mathrm{C}_{\text {org }}$ & $\delta^{13} C_{\text {org }}$ & ${ }^{14} \mathrm{C}_{\text {org }}$ act. & ${ }^{14} \mathrm{C}_{\text {org }}$ age & $\mathrm{C}_{\text {in }}$ & $\delta^{13} C_{\text {in }}$ & ${ }^{14} C_{\text {in }}$ act. & ${ }^{14} \mathrm{C}_{\text {in }}$ age \\
\hline HAM-2552 & $0-2$ & 8.1 & 0.35 & -25.0 & $93.99 \pm 0.49$ & $495 \pm 40$ & 2.30 & -8.9 & $22.31 \pm 0.29$ & $12,050 \pm 100$ \\
\hline HAM-2553 & $2-4$ & 8.1 & 0.31 & -20.7 & $75.50 \pm$ & $2330 \pm 140$ & 2.58 & -9.4 & $41.03 \pm 0.55$ & $7160 \pm 110$ \\
\hline HAM-2554 & $4-6$ & 8.0 & 0.29 & -20.2 & $106.24 \pm 0.61$ & Modern & 2.62 & -11.9 & $37.64 \pm 0.32$ & $7850 \pm 70$ \\
\hline HAM-2555 & $6-8$ & 8.1 & 0.30 & -19.6 & $106.09 \pm 0.52$ & Modern & 2.56 & -- & $35.16 \pm 0.32$ & $8400 \pm 70$ \\
\hline HAM-2556 & $8-10$ & 8.2 & 0.36 & -21.2 & $94.08 \pm 0.78$ & $550 \pm 70$ & 2.61 & -9.8 & $33.57 \pm 0.78$ & $8770 \pm 120$ \\
\hline HAM-2557 & $10-12$ & 8.2 & 0.37 & -20.3 & $110.32 \pm 0.61$ & Modern & 2.26 & -9.7 & $43.21 \pm 0.34$ & $6740 \pm 60$ \\
\hline HAM-2558 & $12-14$ & 8.1 & 0.37 & -23.8 & $105.41 \pm 0.52$ & Modern & 2.68 & -9.8 & $36.05 \pm 0.32$ & $8200 \pm 70$ \\
\hline HAM-2559 & $14-16$ & 8.0 & 0.38 & -19.6 & $101.64 \pm 0.77$ & Modern & 2.46 & -9.6 & $35.55 \pm 0.32$ & $8310 \pm 70$ \\
\hline HAM-2560 & $16-18$ & 8.1 & 0.41 & -19.8 & $101.64 \pm 0.51$ & Modern & 2.37 & -9.7 & $36.40 \pm 0.32$ & $8120 \pm 70$ \\
\hline HAM-2561 & $18-20$ & 8.0 & 0.40 & -18.9 & $102.99 \pm 0.60$ & Modern & 2.36 & -9.9 & $33.63 \pm 0.38$ & $8750 \pm 90$ \\
\hline HAM-2562 & $20-22$ & 8.0 & 0.36 & -23.5 & $103.77 \pm 0.59$ & Modern & 2.47 & -9.7 & $38.53 \pm 0.40$ & $7660 \pm 80$ \\
\hline HAM-2563 & $22-24$ & 8.1 & 0.31 & -21.2 & $103.44 \pm 0.58$ & Modern & 2.50 & -9.5 & $46.21 \pm 0.59$ & $6200 \pm 100$ \\
\hline HAM-2564 & $24-26$ & 8.0 & 0.34 & -21.8 & $103.82 \pm 0.61$ & Modern & 2.38 & -8.8 & $40.32 \pm 0.40$ & $7300 \pm 80$ \\
\hline HAM-2565 & $26-28$ & 7.9 & 0.32 & -22.2 & $80.31 \pm 0.48$ & $1805 \pm 45$ & 2.16 & -9.4 & $38.52 \pm 0.33$ & $7660 \pm 70$ \\
\hline HAM-2566 & $28-30$ & 7.8 & 0.32 & -21.1 & $100.26 \pm 0.83$ & Modern & 2.37 & -9.6 & $42.59 \pm 0.56$ & $6860 \pm 110$ \\
\hline HAM-2567 & $30-32$ & 8.2 & 0.31 & -22.7 & $90.37 \pm 0.66$ & $850 \pm 60$ & 2.22 & -9.7 & $38.95 \pm 0.33$ & $7570 \pm 70$ \\
\hline HAM-2568 & $32-36$ & 8.0 & 0.31 & -18.7 & $=0.57$ & $920 \pm 50$ & 2.39 & -9.4 & $37.71 \pm 0.40$ & $7830 \pm 80$ \\
\hline HAM-2569 & $36-40$ & 7.8 & 0.30 & -17.6 & $92.88 \pm 0.61$ & 710 & 2.46 & -9.9 & $37.67 \pm 0.84$ & $=170$ \\
\hline 2570 & $40-44$ & 7.8 & 0.29 & -16.6 & $86.43 \pm 0.47$ & $1310 \pm 40$ & 2.39 & -9.9 & $36.82 \pm 0.39$ & 803 \\
\hline HAM-2571 & $44-48$ & 8.0 & 0.30 & -19.3 & $89.35 \pm 0.47$ & $995 \pm 40$ & 2.56 & -9.7 & $35.83 \pm 0.39$ & 80 \\
\hline HAM-2572 & $48-52$ & 8.2 & 0.27 & -22.4 & $92.29 \pm 0.48$ & $685 \pm 40$ & 2.54 & -10.3 & $36.80 \pm 0.51$ & $8030 \pm 110$ \\
\hline HAM-2573 & $52-56$ & 8.1 & 0.20 & -18.0 & $86.24 \pm 0.65$ & $1300 \pm 60$ & 2.68 & -9.8 & $37.07 \pm 0.72$ & 150 \\
\hline HAM & $56-60$ & 7.9 & 0.27 & -16.4 & -- & -- & 2.47 & -9.6 & $=0.32$ & 70 \\
\hline HAM-2575 & $60-64$ & 8.1 & 0.28 & -16.3 & $83.54 \pm 0.52$ & $1585 \pm 50$ & 2.42 & -9.4 & 0.32 & $=70$ \\
\hline HAM-2576 & $64-68$ & 8.1 & 0.15 & -16.8 & $79.04 \pm 0.73$ & $2020 \pm 70$ & 2.60 & -9.6 & 0.43 & $=110$ \\
\hline HAM-2577 & $68-72$ & 8.3 & 0.24 & -16.0 & $81.84 \pm 0.67$ & $1760 \pm 70$ & 2.78 & -9.5 & \pm 0.81 & 150 \\
\hline HAM & $72-76$ & 8.2 & 0.25 & -17.3 & $81.78 \pm 0.52$ & $1740 \pm 50$ & 2.43 & -9.5 & $37.71 \pm 0.38$ & 80 \\
\hline HAM-2579 & $76-80$ & 8.3 & 0.25 & -16.4 & $79.72 \pm 0.50$ & $1960 \pm 50$ & 2.51 & -9.6 & $30.36 \pm 0.31$ & 958 \\
\hline HAM-2580 & $80-84$ & 8.3 & 0.18 & -17.1 & $77.91 \pm 0.69$ & \pm 70 & 2.61 & -9.7 & 0.79 & 120 \\
\hline HAM-2581 & $84-88$ & 8.1 & 0.22 & -14.8 & $78.34 \pm 0.62$ & $2130 \pm 60$ & 2.48 & -9.5 & $3 \pm 0.37$ & 100 \\
\hline HAM & $88-92$ & 8.3 & 0.16 & -16.0 & $77.32 \pm 0.68$ & $2210 \pm 70$ & 2.51 & -9.5 & $30.40 \pm 0.54$ & 140 \\
\hline HAN & $92-96$ & 8.3 & 0.20 & -15.6 & $77.34 \pm 0.72$ & $2220 \pm 70$ & 2.52 & -9.3 & $27.08 \pm 0.50$ & $10,490 \pm 150$ \\
\hline HAM & $96-100$ & 8.3 & 0.23 & -18.0 & $74.12 \pm 0.46$ & 50 & 2.44 & -9.5 & -- & -- \\
\hline HAM-2585 & $100-104$ & 8.4 & 0.17 & -20.3 & $71.32 \pm 0.63$ & 2790 & 2.58 & -8.9 & -- & -- \\
\hline HAM-2586 & $104-108$ & 8.5 & 0.23 & -18.0 & $65.78 \pm 0.47$ & $3480 \pm 60$ & 2.54 & -8.7 & $20.58 \pm 0.34$ & $0 \pm 130$ \\
\hline HAM-2587 & $108-112$ & 8.5 & 0.20 & -13.0 & $56.45 \pm 1.75$ & $4790 \pm 240$ & 2.56 & -8.8 & $17.16 \pm 0.47$ & 230 \\
\hline HAM-2588 & $112-116$ & 8.2 & 0.19 & -15.6 & $43.09 \pm 0.56$ & $6920 \pm 110$ & 2.63 & -8.7 & $15.70 \pm 0.32$ & $14,870 \pm 160$ \\
\hline HAM-2589 & $116-120$ & 8.4 & 0.14 & -14.2 & $44.64 \pm 0.57$ & $6650 \pm 100$ & 2.45 & -9.1 & $18.33 \pm 0.28$ & $13,630 \pm 120$ \\
\hline HAM-2590 & $120-124$ & 8.4 & 0.18 & -13.8 & $51.25 \pm 0.65$ & 100 & 2.66 & -8.6 & $12.14 \pm 0.45$ & $16,940 \pm 290$ \\
\hline HAM-2591 & $124-128$ & 8.3 & 0.16 & -14.3 & $41.87 \pm 1.29$ & $7170 \pm 250$ & 2.49 & -9.5 & $10.96 \pm 0.48$ & $17,760 \pm 380$ \\
\hline HAM-2592 & $128-132$ & 8.5 & 0.11 & -12.1 & $37.85 \pm 0.39$ & $8010 \pm 80$ & 2.22 & -7.6 & $6.32 \pm 0.57$ & $22,180 \pm 450$ \\
\hline HAM-2593 & $132-136$ & 8.5 & 0.18 & -13.5 & $43.82 \pm 0.94$ & $6810 \pm 110$ & 2.23 & -8.6 & $7.89 \pm 0.39$ & $20,400 \pm 280$ \\
\hline HAM-2594 & $136-140$ & 8.3 & 0.15 & -12.0 & $44.13 \pm 1.59$ & $6780 \pm 290$ & 2.20 & -9.1 & $24.33 \pm 0.29$ & $11,350 \pm 90$ \\
\hline HAM-2595 & $140-144$ & 8.3 & 0.18 & -12.0 & $21.95 \pm 0.35$ & $12,390 \pm 120$ & 2.18 & -8.4 & $5.31 \pm 0.97$ & $23,580 \pm 1370$ \\
\hline HAM-2596 & $144-148$ & 8.3 & 0.17 & -12.6 & $39.80 \pm 0.47$ & $7600 \pm 90$ & 1.93 & -8.9 & $7.30 \pm 0.43$ & $21,020 \pm 500$ \\
\hline HAM-2597 & $148-152$ & 8.3 & 0.15 & -13.4 & $17.07 \pm 0.33$ & $4,390 \pm 150$ & 2.01 & -8.6 & $5.87 \pm 0.29$ & $22,780 \pm 390$ \\
\hline HAM-2598 & $152-156$ & 8.3 & 0.20 & -14.3 & -- & -- & 1.79 & -7.9 & $6.51 \pm 0.36$ & $21,940 \pm 430$ \\
\hline HAM-2599 & $156-160$ & 8.4 & 0.16 & -13.2 & $34.59 \pm 0.49$ & $8720 \pm 110$ & 1.82 & -8.6 & $6.09 \pm 0.42$ & $22,480 \pm 550$ \\
\hline HAM-2600 & $160-164$ & -. & 0.23 & -13.2 & $21.49 \pm 3.00$ & $12,540 \pm 1030$ & 1.82 & -8.7 & $5.26 \pm 0.55$ & $23,660 \pm 610$ \\
\hline
\end{tabular}


TABLE 10. Qedma (Continued)

\begin{tabular}{lcccccccccc}
\hline Lab code & $\begin{array}{c}\text { Depth } \\
(\mathrm{cm})\end{array}$ & $\mathrm{pH}$ & $\mathrm{C}_{\text {org }}$ & $\delta^{13} \mathrm{C}_{\text {org }}$ & ${ }^{14} \mathrm{C}_{\text {org }}$ act. & ${ }^{14} \mathrm{C}_{\text {org }}$ age & $\mathrm{C}_{\text {in }}$ & $\delta^{13} \mathrm{C}_{\text {in }}$ & ${ }^{14} \mathrm{C}_{\text {in }}$ act. & ${ }^{14} \mathrm{C}_{\text {in }}$ age \\
\hline HAM-2601 & $164-168$ & 8.3 & 0.26 & -13.0 & $26.58 \pm 0.35$ & $10,840 \pm 110$ & 1.77 & -8.8 & $4.25 \pm 0.29$ & $25,370 \pm 540$ \\
HAM-2602 & $168-172$ & 8.4 & 0.36 & -13.4 & $33.14 \pm 0.52$ & $9060 \pm 130$ & 1.67 & -9.2 & $5.61 \pm 0.30$ & $23,140 \pm 430$ \\
HAM-2603 & $172-176$ & 8.4 & 0.09 & -14.1 & $36.48 \pm 1.01$ & $8280 \pm 220$ & 1.90 & -9.8 & $6.72 \pm 0.43$ & $21,690 \pm 530$ \\
HAM-2604 & $176-180$ & 8.4 & 0.14 & -13.0 & $28.79 \pm 0.43$ & $10,200 \pm 120$ & 1.55 & -9.1 & $6.75 \pm 0.25$ & $21,650 \pm 290$ \\
HAM-2605 & $180-184$ & 8.3 & 0.10 & -14.0 & $25.79 \pm 0.48$ & $11,060 \pm 150$ & 1.97 & -8.8 & $4.09 \pm 0.45$ & $25,680 \pm 820$ \\
HAM-2606 & $184-188$ & 8.4 & 0.12 & -13.7 & $23.31 \pm 0.49$ & $11,888 \pm 170$ & 1.93 & -8.8 & - & - \\
HAM-2607 & $188-192$ & 8.4 & 0.21 & -13.9 & $23.63 \pm 0.49$ & $11,770 \pm 170$ & 1.76 & -9.6 & $3.46 \pm 0.42$ & $27,020 \pm 1080$ \\
HAM-2608 & $192-196$ & 8.3 & 0.18 & -14.0 & $22.68 \pm 0.34$ & $12,100 \pm 120$ & 1.64 & -10.4 & $3.71 \pm 0.42$ & $26,460 \pm 980$ \\
HAM-2609 & $196-200$ & 8.4 & 0.26 & -15.0 & $18.85 \pm 0.47$ & $13,570 \pm 200$ & 1.45 & -10.0 & $3.06 \pm 0.42$ & $28,010 \pm 1100$ \\
HAM-2610 & $200-210$ & 8.2 & 0.21 & -16.1 & $21.68 \pm 0.28$ & $12,420 \pm 100$ & 1.56 & -10.2 & $1.76 \pm 0.23$ & $32,450 \pm 1040$ \\
HAM-2611 & $210-220$ & 8.3 & 0.23 & -16.3 & -- & -- & 1.47 & -11.3 & - & - \\
HAM-2612 & $220-230$ & 8.4 & 0.15 & -15.3 & $21.54 \pm 0.82$ & $12,490 \pm 300$ & 1.48 & -11.1 & -- & - \\
HAM-2613 & $230-240$ & 8.3 & 0.08 & -14.9 & $21.20 \pm 0.71$ & $12,420 \pm 270$ & 1.58 & -10.8 & - & - \\
HAM-2614 & $240-250$ & 8.3 & 0.17 & -13.8 & $30.89 \pm 0.73$ & $9620 \pm 190$ & 1.84 & -10.4 & $1.77 \pm 0.41$ & $32,410 \pm 1920$ \\
\hline
\end{tabular}

The texture is characterized by $21.7 \%$ sand, $25.4 \%$ silt and $52.9 \%$ clay (Andresen 1987). The clay fraction consists mostly of montmorillonite (ca. 57\%), kaolinite (ca. 21\%) and illite (ca. 13\%), besides $3 \%$ each of calcite, free oxides and quartz ( $\mathrm{Gal}$ et al. 1974).

\section{Patancheru, Profile P}

\section{Profile-Related Data}

Location (longitude, latitude):

Location (country, next city or village):

Soil order and type (FAO classification):

Soil order and type (USDA classification):

Soil order and type (local classification):

Parent material:

Mean annual temperature:

Annual rainfall:

Vegetation and land use:

Site description:

The profile is on the ICRISAT farm, $c a .500 \mathrm{~m}$ southeast of the Rhodustalf profile. Like this profile, it had been prepared for the International Benchmark Sites Network for Agrotechnology Transfer (IBSNAT). A comparable site nearby was later used within the decomposition studies with ${ }^{14} \mathrm{C}$ labeled groundnut straw by Singer (1993). The vertisols are very homogeneous over the ICRISAT farm area.

Date of sampling:

Date of measurement:

Collector:

Submitter:

Submitter's comment:

1983

$1988 / 1989$

\section{Becker-Heidmann}

Becker-Heidmann

The Vertisol differs from the Alfisol in some ways; it contains more clay and silt, but has no argillic horizon. The $\mathrm{pH}$ is $0.5-2$ higher, because $\mathrm{CaCO}_{3}$ content is about as high as the organic carbon content in the topsoil and even higher in the subsoil. Inorganic carbon is unhomogeneously distributed, increasing slightly from the top to the AC-C boundary and decreasing in horizon $\mathrm{C}$ with depth. Organic $\mathrm{C}$ decreases only slightly in the Ap, remains constant ca. $0.4 \%$ from $\mathrm{A} 1$ through $\mathrm{A} 4$, and decreases in $\mathrm{AC}$ down to $0.2 \%$ in $\mathrm{C}$. With ${ }^{14} \mathrm{C}$ activity generally below $80 \% \mathrm{NBS}$, there is very little recent carbon in the soil. As usual, the ${ }^{14} \mathrm{C}$ activity decreases in Ap and below A14, reaching values of $40 \%$ comparable to the Alfisol. The $\delta^{13} \mathrm{C}$ value of organic 
carbon is higher than expected for the $C_{3}$ plants grown in this soil, and the $\delta^{13} \mathrm{C}$ value of the carbonate is lower than primary (marine) carbonate. Therefore, an exchange of organic and inorganic carbon in the soil via $\mathrm{HCO}_{3}^{-}$in solution/dissolution processes is most probable. The decrease of the ${ }^{14} \mathrm{C}$ activity and the increase of ${ }^{14} \mathrm{C}$ age, respectively, of organic carbon is generally very low, noteworthy only in the top $10 \mathrm{~cm}$ as an indicator of decomposition, and below $90 \mathrm{~cm}$, which corresponds to the maximum depth of cracks due to the self-mulching effect. The typical swelling and shrinking cracks as well as the slickensides in the Vertisol were found down to a depth of $60-90 \mathrm{~cm}$, corresponding to a constant ${ }^{14} \mathrm{C}$ age, while the ${ }^{14} \mathrm{C}$ age rises below $90 \mathrm{~cm}$ depth. The increase of the $\delta^{13} \mathrm{C}$ value of the inorganic carbon to $-2 \%$ within the $\mathrm{AC}$ means that it consists mainly of primary carbonate here, not influenced by biogenic processes.

Comment on both the Alfisol (R) and the Vertisol (P) profile:

The results of the two ICRISAT soils, concerning decomposition and fixation of organic matter, correspond not only to the abovementioned results of decomposition experiments by Singer (1993), but also to biomass determinations (organic C, Chloroform and fumigize method) by Sahrawat (personal communication). He found more biomass in the Alfisol than in the Vertisol, i.e., the decomposition is more intensive in the Alfisol than in the Vertisol.

Literature references:

Becker-Heidmann (1990); Murthy (1982)

\section{Description of Profile $P$ at Patancheru}

\begin{tabular}{cll} 
Depth $(\mathrm{cm})$ & Horizon & Description \\
\hline $0-20$ & Ap & Very dark grayish brown (10YR 3/2), common roots, lime \\
$20-40$ & A12 & Very dark grayish brown (10YR 3/2), common roots, lime \\
$40-60$ & A13 & Very dark gray (10YR 3/1), very few roots, lime concretions \\
$60-90$ & A14 & Very dark gray (10YR 3/1), very few roots, lime concretions \\
$90-130$ & AC & Very dark gray (10YR 3/1), darkbrown (10YR 3/3) mottles \\
$130-180+$ & C & Yellowish to olive brown (2.5Y 5/4), basaltic alluvium
\end{tabular}

TABLE 11. Patancheru, Profile P

\begin{tabular}{|c|c|c|c|c|c|c|c|c|c|c|}
\hline Lab code & $\begin{array}{c}\text { Depth } \\
(\mathrm{cm})\end{array}$ & $\mathrm{pH}$ & $\mathrm{C}_{\text {org }}$ & $\delta^{13} C_{\text {org }}$ & ${ }^{14} \mathrm{C}_{\text {org }}$ act. & ${ }^{14} \mathrm{C}_{\text {org }}$ age & $\mathrm{C}_{\text {in }}$ & $\delta^{13} C_{\text {in }}$ & ${ }^{14} \mathrm{C}_{\text {in }}$ act. & ${ }^{14} \mathrm{C}_{\text {in }}$ age \\
\hline HAM-2054 & $0-2$ & 7.4 & 0.53 & -15.5 & $81.81 \pm 0.53$ & $1615 \pm 50$ & 0.45 & -6.7 & $67.86 \pm 0.65$ & $3110 \pm 80$ \\
\hline HAM-2055 & $2-4$ & 7.4 & 0.57 & -15.2 & 70.3 & $2830 \pm 60$ & 0.38 & -7.5 & 6 & 30 \\
\hline HAM-2056 & $4-6$ & 7.4 & 0.52 & -14.9 & $73.57 \pm 0.56$ & $2470 \pm 60$ & 0.45 & -7.0 & $68.52 \pm 0.66$ & 80 \\
\hline HAM-2057 & $6-8$ & 7.4 & 0.46 & -14.8 & $74.10 \pm 0.50$ & $2410 \pm 50$ & 0.32 & -6.7 & $67.50 \pm 0$ & 80 \\
\hline HAM-2058 & $8-10$ & 7.4 & 0.50 & -14.9 & $73.64 \pm 0.50$ & $2460 \pm 60$ & 0.48 & -6.7 & 66.3 & 80 \\
\hline HAM-2059 & $10-12$ & 7.4 & 0.52 & -14.3 & $71.86 \pm 0.50$ & \pm 50 & 0.37 & -6.4 & $64.80 \pm 1.15$ & $3490 \pm 140$ \\
\hline 2060 & 12 & 7.4 & 0.44 & -14 & 72. & $=60$ & 0.42 & -6.8 & -- & -- \\
\hline HAM-2061 & $14-16$ & 7.4 & 0.53 & -14.7 & 71. & \pm 50 & 0.46 & -6.9 & $64.35 \pm 0.64$ & $3540 \pm 80$ \\
\hline 2062 & $16-18$ & 7.4 & 0.43 & -14.8 & $72.13 \pm 0.51$ & 262 & 0.53 & -7.0 & $64.69=$ & $3500 \pm 80$ \\
\hline 2063 & $18-20$ & 7.4 & 0.44 & -14.8 & $63.08 \pm 0.47$ & $3700 \pm 60$ & 0.58 & -6.9 & $70.94 \pm 0.70$ & $2760 \pm 80$ \\
\hline HAM-2064 & $20-22$ & 7.4 & 0.45 & -14.8 & $70.34 \pm 0.49$ & $2830 \pm 60$ & 0.54 & -7.2 & $65.13 \pm 0.63$ & $3440 \pm 80$ \\
\hline 2065 & $22-24$ & 7.4 & 0.46 & -14.9 & $73.50 \pm 0.50$ & $2470 \pm 60$ & 0.35 & -7.4 & $45.55 \pm 0.61$ & $6320 \pm 110$ \\
\hline 2066 & $24-26$ & 7.4 & 0.45 & -14.6 & $32.20 \pm 0.38$ & $9100 \pm 90$ & 0.50 & -6.9 & $64.04 \pm$ & 3580 \\
\hline 2067 & $26-28$ & 7.5 & 0.47 & -14.6 & \pm 0.49 & 303 & 0.45 & -7.3 & 64. & 60 \\
\hline 2068 & $28-30$ & 7.5 & 0.46 & -14.6 & $68.13 \pm 0.49$ & 3080 & 0.51 & -6.7 & 63.01 & 90 \\
\hline 2069 & $30-32$ & 7.5 & 0.46 & -14.4 & 69. & 60 & 0.42 & -7.0 & 63.88 & $3600 \pm 80$ \\
\hline 070 & $32-34$ & 7.5 & 0.46 & -14.8 & $64.70 \pm 0.47$ & $3500 \pm 60$ & 0.34 & -7.4 & $64.38 \pm 0.63$ & \pm 80 \\
\hline HAM-2071 & $34-36$ & 7.4 & 0.47 & -14.6 & $68.85 \pm 0.49$ & $3000 \pm 60$ & 0.42 & -6.9 & $64.42 \pm 0.65$ & $3530 \pm 80$ \\
\hline-2072 & $36-38$ & 7.6 & 0.44 & -14.5 & $74.24 \pm 1.08$ & $2390 \pm 120$ & 0.50 & -6.8 & $61.09 \pm 0.46$ & $3960 \pm 60$ \\
\hline HAM-2073 & $38-40$ & 7.6 & 0.48 & -14.5 & $71.45 \pm 0.50$ & $2700 \pm 60$ & 0.34 & -7.2 & $62.32 \pm 0.63$ & $3800 \pm 80$ \\
\hline HAM-2074 & $40-42$ & 7.6 & 0.46 & -14.4 & $75.49 \pm 0.51$ & $2260 \pm 50$ & 0.48 & -6.5 & $60.79 \pm 0.46$ & $4000 \pm 60$ \\
\hline HAM-2075 & $42-44$ & 7.6 & 0.48 & -14.5 & $73.13 \pm 0.60$ & 70 & 0.38 & -6.8 & $45.47 \pm 0.70$ & $6330 \pm 120$ \\
\hline-2076 & $44-46$ & 7.6 & 0.48 & -14.7 & $75.56 \pm 0.68$ & $2250 \pm 70$ & 0.46 & -6.6 & $59.66 \pm 0.62$ & $4150 \pm 80$ \\
\hline $\mathrm{H}$ & 4 & 7. & 0.48 & r & 68.3 & 3060 & 0.50 & -6.8 & 63.1 & $3700 \pm 80$ \\
\hline
\end{tabular}


TABLE 11. Patancheru, Profile P (Continued)

\begin{tabular}{|c|c|c|c|c|c|c|c|c|c|c|}
\hline Lab code & $\begin{array}{l}\text { Depth } \\
\text { (cm) }\end{array}$ & $\mathrm{pH}$ & $\mathrm{C}_{\text {org }}$ & $\delta^{13} C_{\text {org }}$ & ${ }^{14} \mathrm{C}_{\text {org }}$ act. & ${ }^{14} \mathrm{C}_{\text {org }}$ age & $\mathrm{C}_{\text {in }}$ & $\delta^{13} C_{\text {in }}$ & ${ }^{14} \mathrm{C}_{\text {in }}$ act. & ${ }^{14} \mathrm{C}_{\text {in }}$ age \\
\hline HAM-2078 & $48-50$ & 7.6 & 0.48 & -14.5 & $74.30 \pm 0.48$ & $2390 \pm 50$ & 0.44 & -6.7 & $61.39 \pm 0.62$ & $3920 \pm 80$ \\
\hline HAM-2079 & $50-52$ & 7.7 & 0.46 & -14.4 & $28.47 \pm 0.50$ & $10,090 \pm 140$ & 0.36 & -6.9 & $59.51 \pm 0.61$ & $4170 \pm 80$ \\
\hline HAM-2080 & $52-54$ & 7.7 & 0.44 & -14.6 & $68.24 \pm 1.26$ & $3070 \pm 150$ & 0.41 & -6.9 & $10.23 \pm 0.46$ & $18,310 \pm 360$ \\
\hline HAM-2081 & & 7.7 & 0.46 & -14.4 & $77.24 \pm 0.69$ & $2070 \pm 70$ & 0.51 & -7.0 & $54.86 \pm 0.60$ & $4820 \pm 90$ \\
\hline HAM-2082 & $56-58$ & 7.7 & 0.42 & -14.7 & $81.61 \pm 0.61$ & $1630 \pm 60$ & 0.55 & -6.9 & $58.33 \pm 0.62$ & $4330 \pm 90$ \\
\hline HAM-2083 & $58-60$ & 7.7 & 0.43 & -14.3 & -- & -- & 0.61 & -7.7 & -- & -- \\
\hline HAM-2084 & $60-62$ & 7.7 & 0.46 & -14.9 & $83.54 \pm 0.61$ & $1440 \pm 60$ & 0.43 & -7.4 & $61.64 \pm 0.63$ & $3890 \pm 80$ \\
\hline HAM-2085 & $62-64$ & 7.8 & 0.47 & -14.3 & $76.47 \pm 0.95$ & $2160 \pm 100$ & 0.52 & -6.9 & $60.14 \pm 0.63$ & $4080 \pm 80$ \\
\hline HAM-2086 & $64-66$ & 7.8 & 0.46 & -14.3 & $71.33 \pm 0.62$ & $2710 \pm 70$ & 0.37 & -6.9 & $57.69 \pm 0.61$ & $4420 \pm 90$ \\
\hline HAM-2087 & $66-68$ & 7.7 & 0.46 & -14.2 & $75.82 \pm 2.37$ & $2220 \pm 250$ & 0.54 & -6.8 & $23.79 \pm 0.48$ & $11,530 \pm 160$ \\
\hline HAM-2088 & $68-70$ & 7.7 & 0.45 & -14.3 & $57.40 \pm 0.62$ & $4460 \pm 90$ & 0.57 & -6.7 & $61.88 \pm 0.63$ & $3860 \pm 80$ \\
\hline HAM-2089 & $70-72$ & 7.7 & 0.42 & -14.3 & $30.85 \pm 0.52$ & $9450 \pm 140$ & 0.62 & -6.7 & $48.47 \pm 0.58$ & $5820 \pm 100$ \\
\hline HAM-2090 & $72-74$ & 7.8 & 0.44 & -14.2 & $68.68 \pm 0.65$ & $3020 \pm 80$ & 0.61 & -6.5 & $59.16 \pm 0.62$ & $4220 \pm 80$ \\
\hline HAM-2091 & $74-76$ & 7.9 & 0.46 & -14.4 & $67.73 \pm 3.01$ & $=360$ & 0.42 & -6.7 & $57.22 \pm 0.46$ & $4480 \pm 70$ \\
\hline HAM-2092 & $76-78$ & 7.8 & 0.48 & -14.2 & $65.26 \pm 0.50$ & $3430 \pm 60$ & 0.40 & -6.7 & $61.24 \pm 0.77$ & $3940 \pm 100$ \\
\hline HAM-2093 & $78-80$ & 7.8 & 0.44 & -14.2 & $70.69 \pm 0.93$ & $2790 \pm 110$ & 0.61 & -6.3 & $58.11 \pm 0.61$ & $4360 \pm 80$ \\
\hline HAM-2094 & $80-82$ & 7.8 & 0.47 & -14.0 & $66.09 \pm 0.64$ & $3330 \pm 80$ & 0.48 & -6.3 & 62 & 90 \\
\hline HAM-2095 & $82-84$ & 7.9 & 0.46 & -14.7 & $75.97 \pm 0.68$ & $2210 \pm 70$ & 0.43 & -7.0 & $60.39 \pm 0.62$ & 80 \\
\hline HAI & $84-86$ & 7.9 & 0.46 & -14.0 & $70.78 \pm 0.66$ & $2780 \pm 80$ & 0.63 & -6.3 & $55.57 \pm 0.61$ & $4720 \pm 90$ \\
\hline HAN & $86-88$ & 8.0 & 0.44 & -14.2 & $47.85 \pm 0.42$ & $5920 \pm 70$ & 0.44 & -6.4 & $23.35 \pm 0.74$ & $11,680 \pm 250$ \\
\hline HAM-2098 & $88-90$ & 7.9 & 0.43 & -14.2 & $67.08 \pm 0.66$ & $3210 \pm 80$ & 0.42 & -6.8 & $55.92 \pm 0.60$ & $4670 \pm 90$ \\
\hline HAM-2099 & $90-92$ & 7.9 & 0.44 & -14.4 & $60.17 \pm 0.53$ & $4080 \pm 70$ & 0.42 & -6.4 & $52.32 \pm 3.68$ & $5200 \pm 560$ \\
\hline HAM-2100 & 92-94 & 7.9 & 0.39 & -14.2 & $66.97 \pm 0.65$ & 3220 & 0.63 & 6.1 & $53.25 \pm 0.60$ & $5060 \pm 90$ \\
\hline HAM & $94-96$ & 8.0 & 0.40 & -14.0 & $63.48 \pm 0$ & \pm 80 & 0.50 & -5.8 & $48.39 \pm 0.64$ & $5830 \pm 110$ \\
\hline HAM-2102 & $96-98$ & 8.0 & 0.38 & -14.0 & $46.04 \pm 0.57$ & 100 & 0.59 & 5.7 & $50.48 \pm 0.59$ & $5490 \pm 90$ \\
\hline HAM-2103 & $98-100$ & 7.9 & 0.46 & -14.3 & $74.66 \pm 0.67$ & 2350 & 0.40 & -5.9 & $53.12 \pm 0.60$ & $5080 \pm 90$ \\
\hline HAM-2104 & $100-102$ & 8.1 & 0.35 & -14.0 & $60.49 \pm 0.80$ & $4040 \pm 110$ & 0.53 & -5.0 & $47.87 \pm 0.58$ & 5920 \\
\hline HAM-2105 & $102-104$ & 8.0 & 0.35 & -13.8 & -- & -- & 0.41 & -5.4 & -- & 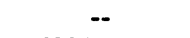 \\
\hline HAM-2106 & $104-106$ & 8.0 & 0.34 & -13.4 & $60.74 \pm 0.56$ & $4000 \pm 70$ & 0.66 & -4.5 & $42.91 \pm 0.41$ & 30 \\
\hline HAM-2107 & $106-108$ & 8.0 & 0.34 & -13.6 & $72.92 \pm 0.83$ & $2540 \pm 90$ & 0.51 & -4.9 & $44.32 \pm 0.57$ & 100 \\
\hline HAM-2108 & $108-110$ & 8.1 & 0.30 & -13.3 & $36.61 \pm 1.60$ & $8070 \pm 350$ & 0.78 & -4.2 & $38.72 \pm 0.54$ & 110 \\
\hline HAN & $110-112$ & 8.0 & 0.33 & -13.9 & $71.89 \pm 0.67$ & $2650 \pm 70$ & 0.56 & -4.9 & $44.66 \pm 0.57$ & 100 \\
\hline HAM-2110 & $112-114$ & 8.0 & 0.32 & -13.8 & $68.01 \pm 0.75$ & $3100 \pm 90$ & 0.50 & -5.2 & $41.70 \pm 0.55$ & $7030 \pm 110$ \\
\hline HAM-2111 & $114-116$ & 8.0 & 0.27 & -13.2 & $59.11 \pm 0.83$ & $4220 \pm 110$ & 0.50 & -4.9 & $37.61 \pm 0.54$ & $7860 \pm 120$ \\
\hline HAM-2112 & $116-118$ & 8.0 & 0.29 & -14.5 & $47.68 \pm 0.58$ & 100 & 0.48 & -4.7 & $2.83 \pm 0.40$ & $28,640 \pm 1150$ \\
\hline HAM-2113 & $118-120$ & 8.0 & 0.27 & -13.5 & $70.86 \pm 0.86$ & 100 & 0.50 & -4.4 & $41.38 \pm 0.55$ & $7090 \pm 110$ \\
\hline HAM-2114 & $120-122$ & 8.0 & 0.22 & -13.2 & 63.48 & 100 & 0.46 & -4.6 & $38.99 \pm 0.58$ & $7570 \pm 120$ \\
\hline HAM-2115 & $122-124$ & 8.1 & 0.20 & -12.4 & $58.69 \pm 0.46$ & 428 & 0.68 & -3.9 & $59 \pm 0.51$ & $9780 \pm 140$ \\
\hline HAM-2116 & $124-126$ & 8.0 & 0.20 & -13.3 & $71.37 \pm 0.57$ & 271 & 0.82 & -3.4 & $40.91 \pm 0.55$ & $=110$ \\
\hline HAM-2117 & $126-128$ & 8.1 & 0.22 & -11.1 & $61.34 \pm 0.86$ & 110 & 1.04 & -2.3 & $30.68 \pm 0.51$ & $9490 \pm 130$ \\
\hline HAM-2118 & $128-130$ & 8.0 & 0.20 & -11.3 & $51.09 \pm 0.49$ & 5390 & 1.26 & -2.2 & $22.36 \pm 0.49$ & $12,030 \pm 180$ \\
\hline HAM-2119 & $130-132$ & 8.0 & 0.22 & -13.0 & $68.19 \pm 0.96$ & $3080 \pm 110$ & 0.52 & -3.5 & 33.92 & 120 \\
\hline HAM-2120 & $132-134$ & 8.1 & 0.18 & -12.0 & $67.84 \pm 0.90$ & $3120 \pm 110$ & 0.61 & -3.3 & $30.72 \pm 0.50$ & 130 \\
\hline HAM-2121 & $134-136$ & 8.0 & 0.23 & -12.8 & $40.05 \pm 0.55$ & $7350 \pm 110$ & 0.53 & -3.6 & $28.27 \pm 0.50$ & 140 \\
\hline HAM-2122 & $136-138$ & 8.1 & 0.21 & -12.6 & $63.23 \pm 0.63$ & $3680 \pm 80$ & 0.46 & -3.5 & $27.42 \pm 0.49$ & 10,390 \\
\hline HAM & $138-140$ & 8.1 & 0.18 & -12.4 & $66.13 \pm 0.64$ & $3320 \pm 80$ & 0.58 & -3.3 & $30.10 \pm 0.50$ & $9640 \pm 140$ \\
\hline HAM-2124 & $140-142$ & 8.0 & 0.21 & -14.1 & $70.20 \pm 0.87$ & $2840 \pm 100$ & 0.50 & -3.3 & $30.38 \pm 0.51$ & $9570 \pm 140$ \\
\hline HAM-2125 & $142-144$ & 8.0 & 0.19 & -11.5 & - & -- & 0.95 & -2.7 & -- & -- \\
\hline HAM-2126 & $144-146$ & 8.1 & 0.18 & -11.5 & -- & -- & 0.59 & -3.1 & $29.54 \pm 0.50$ & $9800 \pm 140$ \\
\hline HAM-2127 & $146-148$ & 8.1 & 0.19 & -14.0 & $68.37 \pm 0.67$ & $50 \pm 80$ & 0.67 & -3.6 & $22.27 \pm 0.47$ & $12,070 \pm 170$ \\
\hline HAM-2128 & $148-150$ & 8.0 & 0.21 & -11.6 & $71.00 \pm 1.00$ & $2750 \pm 110$ & 0.77 & -3.3 & $26.37 \pm 0.49$ & $10,710 \pm 150$ \\
\hline HAM-2129 & $150-152$ & 8.1 & 0.21 & -12.5 & - & -- & 0.68 & -2.4 & $20.39 \pm 0.46$ & $12,770 \pm 180$ \\
\hline HAM-2130 & $152-154$ & 8.1 & 0.10 & -11.4 & $58.97 \pm 0.63$ & $4240 \pm 90$ & 0.61 & -2.5 & $19.10 \pm 0.47$ & $13,300 \pm 200$ \\
\hline HAM-2131 & $154-156$ & 8.1 & 0.17 & -11.4 & $65.34 \pm 0.67$ & $3420 \pm 80$ & 0.42 & -3.0 & $28.37 \pm 0.51$ & $10,120 \pm 140$ \\
\hline HAM-2132 & $156-158$ & 8.1 & 0.17 & -11.2 & $65.77 \pm 0.83$ & $3370 \pm 100$ & 0.61 & -2.6 & $21.54 \pm 0.48$ & $12,330 \pm 180$ \\
\hline HAM-2133 & $158-160$ & 8.1 & 0.18 & -12.4 & $60.56 \pm 0.48$ & $4030 \pm 60$ & 0.35 & -3.4 & $20.06 \pm 0.47$ & $12,900 \pm 190$ \\
\hline
\end{tabular}


TABLE 11. Patancheru, Profile P (Continued)

\begin{tabular}{|c|c|c|c|c|c|c|c|c|c|c|}
\hline Lab code & $\begin{array}{c}\text { Depth } \\
\text { (cm) }\end{array}$ & $\mathrm{pH}$ & $\mathrm{C}_{\text {org }}$ & $\delta^{13} C_{\text {org }}$ & ${ }^{14} \mathrm{C}_{\text {org }}$ act. & ${ }^{14} \mathrm{C}_{\text {org }}$ age & $C_{\text {in }}$ & $\delta^{13} C_{i n}$ & ${ }^{14} \mathrm{C}_{\text {in }}$ act. & ${ }^{14} C_{\text {in }}$ age \\
\hline HAM-2134 & $160-162$ & 8.1 & 0.17 & -11.2 & $61.33 \pm 0.66$ & $3930 \pm 90$ & 0.33 & -2.9 & $18.60 \pm 0.47$ & $13,510 \pm 200$ \\
\hline HAM-2135 & $162-164$ & 8.1 & 0.12 & -13.4 & $63.81 \pm 0.65$ & $3610 \pm 80$ & 0.42 & -3.2 & $27.06 \pm 0.50$ & $10,500 \pm 150$ \\
\hline HAM-2136 & $164-166$ & 8.1 & 0.16 & -12.2 & $58.98 \pm 0.90$ & $4240 \pm 120$ & 0.36 & -2.9 & $21.30 \pm 0.47$ & $12,420 \pm 180$ \\
\hline HAM-2137 & $166-168$ & 8.1 & 0.15 & -12.4 & $70.77 \pm 1.06$ & $2780 \pm 120$ & 0.41 & -2.8 & $24.63 \pm 0.49$ & $11,260 \pm 160$ \\
\hline HAM-2138 & $168-170$ & 8.1 & 0.17 & -12.3 & $62.27 \pm 0.89$ & $3810 \pm 120$ & 0.17 & -2.8 & $21.19 \pm 0.48$ & $12,460 \pm 180$ \\
\hline HAM-2139 & $170-172$ & 8.0 & 0.19 & -11.7 & $66.38 \pm 0.91$ & $3290 \pm 110$ & 0.35 & -3.2 & $24.08 \pm 0.48$ & $11,440 \pm 160$ \\
\hline HAM-2140 & $172-174$ & 8.1 & 0.15 & -10.4 & $45.01 \pm 0.64$ & $6410 \pm 110$ & 0.62 & -1.9 & $11.29 \pm 0.44$ & $17,520 \pm 310$ \\
\hline HAM-2141 & $174-176$ & 8.0 & 0.16 & -11.4 & $71.86 \pm 0.96$ & $2650 \pm 110$ & 0.60 & -2.1 & $18.22 \pm 0.47$ & $13,680 \pm 210$ \\
\hline HAM-2142 & $176-178$ & 8.1 & 0.16 & -12.4 & $61.40 \pm 0.70$ & $3920 \pm 90$ & 0.37 & -3.1 & $26.36 \pm 0.49$ & $10,710 \pm 150$ \\
\hline HAM-2143 & $178-180$ & 8.0 & 0.19 & -13.5 & $74.36 \pm 0.71$ & $2380 \pm 80$ & 0.50 & -2.5 & $22.99 \pm 0.48$ & $11,810 \pm 170$ \\
\hline HAM-2144 & $180-182$ & 8.1 & 0.15 & -12.7 & $68.05 \pm 1.06$ & $3090 \pm 130$ & 0.30 & -3.0 & $24.86 \pm 0.48$ & $11,180 \pm 160$ \\
\hline HAM-2145 & 182-184 & 8.0 & 0.16 & -12.5 & $66.20 \pm 1.02$ & $3310 \pm 120$ & 0.40 & -3.1 & $19.08 \pm 0.46$ & $13,310 \pm 200$ \\
\hline HAM-2146 & $184-186$ & 8.1 & 0.15 & -13.0 & $68.34 \pm 0.95$ & $3060 \pm 110$ & 0.29 & -3.0 & $22.85 \pm 0.48$ & $11,860 \pm 170$ \\
\hline
\end{tabular}

Bulk density: 1-1.2 $\mathrm{g} \mathrm{cm}^{-3}$, according to VERMA (1990), cited in Singer (1993).

\section{Patancheru, Profile R}

\section{Profile-Related Data}

Location (longitude, latitude):

Location (country, next city or village):

Soil order and type (FAO classification):

$17^{\circ} 35^{\prime} \mathrm{N}, 78^{\circ} 17^{\prime} \mathrm{E}$

Soil order and type (USDA classification):

India, Patancheru near Hyderabad (ICRISAT)

Chromic Luvisol

Udic Rhodustalf, clayey-skeletal, mixed, nonacid, isohyperthermic

Soil order and type (local classification):

Parent material:

Patancheru series

Mean annual temperature:

Weathered granite-gneiss

Annual rainfall:

$25.8^{\circ} \mathrm{C}$

Vegetation and land use:

$760 \mathrm{~mm}$

Site description:

Rainfed Sorghum, maize, pulses

$540 \mathrm{~m}$ asl

The sampling area is located within the experimental farm of the Institute for Crops Research of the SemiArid Tropics (ICRISAT). The mean soil temperature is $1^{\circ}$ higher than the air temperature. On both soils also decomposition experiments with ${ }^{14} \mathrm{C}$ labeled straw have been conducted (SINGER 1993). The chosen soil profile had been prepared for the International Benchmark Sites Network for Agrotechnology Transfer (IBSNAT).

Date of sampling:

Date of measurement:

1983

Collector:

Submitter:

$1984 / 1985$

Becker-Heidmann

Becker-Heidmann

Submitter's comment:

The C content of the Rhodustalf profile is very low. Only the topmost 2-cm layer and the bottom of the A12 horizon shows a maximum value of $0.8 \%$, probably due to the recently settled grass vegetation. The site had been fallow since 1973, when ICRISAT was founded. The decomposition of SOM then probably led to the decrease of $C$ content from the bottom to the top of the formerly plowed horizon. In the $B$ horizon the $C$ content is decreasing with depth in the normal manner. The increase beneath the $\mathrm{B}$, in the $\mathrm{Cv}$, is according to increasing $\mathrm{CaCO}_{3}$ content.

Concerning the natural ${ }^{14} \mathrm{C}$ activity, four zones can be distinguished in the Patancheru profile. The ${ }^{14} \mathrm{C}$ activity is equal to the atmospheric bomb carbon level only in the topmost $2-\mathrm{cm}$ layer. The ${ }^{14} \mathrm{C}$ activity is nearly 
uniform, as expected, but $<120$ pMC below the first $4 \mathrm{~cm}$ of the plowed horizon. This indicates low annual $\mathrm{C}$ input combined with rapid turnover, in accordance with the low $\mathrm{C}$ content of the soil. This interpretation is strongly supported by experiments of Singer (1993) who found $76 \%$ of incorporated ${ }^{14} \mathrm{C}$-labeled groundnut straw decomposed in a nearby Alfisol after 30 weeks. The $\delta^{13} \mathrm{C}$ value decreases from $-15 \%$ to $-18 \%$. $\delta^{13} \mathrm{C}$ usually is not only more negative but also increases with depth in the topsoil, correlating with $\mathrm{C}_{3}$ plants and the increasing decomposition stage of organic matter with depth (Stout, Goh and Rafter (1981); O'Brien and Stout (1978); Becker-Heidmann and Scharpenseel (1986)). The reason for a different curve in this profile is most probably a recent change in cultivation from pulses $\left(C_{3}\right.$ plants $)$ to sorghum $\left(C_{4}\right)$, as could be clarified by measurements of $\delta^{13} \mathrm{C}$ of the vegetation presently found there (Becker-Heidmann 1989, Table 5.1). Some of the fresh organic substances with higher $\delta^{13} \mathrm{C}$ values move downwards and are added to the older organic matter, which originated in the $\mathrm{C}_{3}$ crops. Below Ap and $\mathrm{A} 1, \delta^{13} \mathrm{C}$ shows a normal pattern. From $\mathrm{A} 12$ to $\mathrm{B} 21 \mathrm{t}$ horizons, the ${ }^{14} \mathrm{C}$ activity decreases continuously until it is $c a .60 \mathrm{pMC}$, corresponding to a rapidly decreasing rejuvenation by translocated carbon. Accordingly, increasing $\delta^{13} \mathrm{C}$ shows a rise in more decomposed organic matter with depth. In the B22t horizon, then, the tendencies of both ${ }^{14} \mathrm{C}$ activity and $\delta^{13} \mathrm{C}$ inflect coincidently with the beginning of the decline of the clay content. We also found the same effect in Alfisols from temperate zone forests, e.g., at Wohldorf and Ohlendorf Forest (see above) (Becker-Heidmann and Scharpenseel 1986).

Because of the low precipitation at Patancheru, little young organic matter enters and moves downwards into the profile. But with decreasing amounts of clay and, consequently, of clay-complexated old organic matter, the influence of young organic matter grows with depth. Greenland (1971) and Theng (1979) found 98\% of the carbon of tropical red soils ( $\mathrm{C}$ content $1.7 \%$ ) bound to clay minerals.

Literature references:

Becker-Heidmann (1989); Murthy et al. (1982)

\section{Description of Profile $R$ at Patancheru}

\begin{tabular}{cll} 
Depth $(\mathrm{cm})$ & Horizon & Description \\
\hline $0-10$ & Ap & Yellowish red (5YR 5/6), many fine roots \\
$10-20$ & A12 & Reddish brown (5YR 4/4), few fine roots \\
$20-30$ & B1 & Reddish brown (2.5YR 4/4), few fine roots \\
$30-48$ & B21t & Dark reddish brown (2.5YR 3/4), few fine roots \\
$48-102$ & B22t & Dark reddish brown (2.5YR 3/4), very few roots \\
$102-146$ & BC & Reddish (2.5YR 5/4) to yellowish brown (10YR 6/4), iron concretions \\
$146-160+$ & C & Yellowish brown (10YR 6/4), weathered granite-gneiss
\end{tabular}

TABLE 12. Patancheru, Profile $R$

\begin{tabular}{lcccccc}
\hline Lab code & Depth $(\mathrm{cm})$ & $\mathrm{pH}$ & $\mathrm{C}$ & $\delta^{13} \mathrm{C}$ & ${ }^{14} \mathrm{C}$ activity & ${ }^{14}$ C age* \\
\hline HAM-1975 & $0-2$ & 6.7 & 0.72 & -14.9 & $114.88 \pm 0.71$ & Modern \\
HAM-1976 & $2-4$ & 6.3 & 0.42 & -16.2 & $101.16 \pm 0.66$ & Modern \\
HAM-1977 & $4-6$ & 6.6 & 0.45 & -16.4 & $107.37 \pm 0.67$ & Modern \\
HAM-1978 & $6-8$ & 6.5 & 0.43 & -16.8 & $105.48 \pm 0.67$ & Modern \\
HAM-1979 & $8-10$ & 6.4 & 0.48 & -16.8 & $107.44 \pm 0.68$ & Modern \\
HAM-1980 & $10-12$ & 6.2 & 0.42 & -17.3 & $104.38 \pm 0.66$ & Modern \\
HAM-1981 & $12-14$ & 6.0 & 0.44 & -17.8 & $99.60 \pm 0.64$ & $150 \pm 50$ \\
HAM-1982 & $14-16$ & 5.9 & 0.56 & -17.6 & $97.42 \pm 0.63$ & $330 \pm 50$ \\
HAM-1983 & $16-18$ & 5.8 & 0.59 & -17.8 & $95.09 \pm 0.62$ & $520 \pm 50$ \\
HAM-1984 & $18-20$ & 5.8 & 0.65 & -17.6 & $92.09 \pm 0.61$ & $780 \pm 50$ \\
HAM-1985 & $20-22$ & 5.7 & 0.60 & -18.0 & $90.52 \pm 0.61$ & $910 \pm 50$ \\
HAM-1986 & $22-24$ & 5.8 & 0.67 & -17.2 & $89.25 \pm 0.60$ & $1040 \pm 50$ \\
HAM-1987 & $24-26$ & 5.8 & 0.58 & -17.3 & $88.94 \pm 0.60$ & $1070 \pm 50$ \\
HAM-1988 & $26-28$ & 5.8 & 0.55 & -17.4 & $87.57 \pm 0.60$ & $1190 \pm 60$
\end{tabular}


TABLE 12. Patancheru, Profile R (Continued)

\begin{tabular}{|c|c|c|c|c|c|c|}
\hline Lab code & Depth $(\mathrm{cm})$ & $\mathrm{pH}$ & $\mathrm{C}$ & $\delta^{13} \mathrm{C}$ & ${ }^{14} \mathrm{C}$ activity & ${ }^{14} \mathrm{C}$ age* \\
\hline HAM-1989 & $28-30$ & 5.9 & 0.55 & -16.9 & $86.81 \pm 0.59$ & $1270 \pm 50$ \\
\hline HAM-1990 & $30-32$ & 5.9 & 0.52 & -16.7 & $86.16 \pm 0.59$ & $1330 \pm 60$ \\
\hline HAM-1991 & $32-34$ & 6.3 & 0.53 & -16.9 & $83.74 \pm 0.62$ & $1560 \pm 60$ \\
\hline HAM-1992 & $34-36$ & 5.9 & 0.50 & -16.9 & $82.79 \pm 0.58$ & $1650 \pm 60$ \\
\hline HAM-1993 & $36-38$ & 6.2 & 0.47 & -16.1 & $78.71 \pm 0.56$ & $2070 \pm 60$ \\
\hline HAM-1994 & $38-40$ & 5.9 & 0.48 & -16.1 & $77.76 \pm 0.56$ & $2170 \pm 60$ \\
\hline HAM-1995 & $40-42$ & 5.9 & 0.52 & -15.2 & $77.37 \pm 0.55$ & $2220 \pm 60$ \\
\hline HAM-1996 & $42-44$ & 5.8 & 0.46 & -15.5 & $73.21 \pm 0.55$ & $2660 \pm 60$ \\
\hline HAM-1997 & $44-46$ & 5.8 & 0.51 & -15.5 & $69.79 \pm 0.53$ & $3040 \pm 60$ \\
\hline HAM-1998 & $46-48$ & 5.7 & 0.53 & -15.4 & $65.51 \pm 0.52$ & $3550 \pm 60$ \\
\hline HAM-1999 & $48-50$ & 5.8 & 0.51 & -15.4 & $65.54 \pm 0.52$ & $3550 \pm 60$ \\
\hline HAM-2000 & $50-52$ & 5.7 & 0.45 & -15.5 & $67.06 \pm 0.52$ & $3360 \pm 60$ \\
\hline HAM-2001 & $52-54$ & 5.8 & 0.54 & -15.3 & $71.06 \pm 0.53$ & $2900 \pm 60$ \\
\hline HAM-2002 & $54-56$ & 5.7 & 0.33 & -15.7 & $62.38 \pm 0.51$ & $3940 \pm 70$ \\
\hline HAM-2003 & $56-58$ & 5.7 & 0.40 & -15.2 & $67.52 \pm 0.52$ & $3310 \pm 60$ \\
\hline HAM-2004 & $58-60$ & 5.7 & 0.44 & -15.3 & $64.39 \pm 0.51$ & $3690 \pm 60$ \\
\hline HAM-2005 & $60-62$ & 5.7 & 0.36 & -15.1 & $63.97 \pm 0.60$ & $3750 \pm 80$ \\
\hline HAM-2006 & $62-64$ & 5.7 & 0.40 & -15.1 & $61.99 \pm 0.59$ & $4000 \pm 80$ \\
\hline HAM-2007 & $64-66$ & 5.8 & 0.43 & -14.9 & $63.38 \pm 0.60$ & $3660 \pm 80$ \\
\hline HAM-2008 & $66-68$ & 5.7 & 0.33 & -14.9 & $61.02 \pm 0.58$ & $3970 \pm 80$ \\
\hline HAM-2009 & $68-70$ & 5.7 & 0.31 & -15.0 & $60.79 \pm 0.58$ & $4160 \pm 80$ \\
\hline HAM-2010 & $70-72$ & 5.8 & 0.32 & -15.4 & $57.88 \pm 0.57$ & $4550 \pm 80$ \\
\hline HAM-2011 & $72-74$ & 5.8 & 0.33 & -15.7 & $60.61 \pm 0.58$ & $4170 \pm 80$ \\
\hline HAM-2012 & $74-76$ & 6.0 & 0.49 & -15.2 & $66.44 \pm 0.60$ & $3440 \pm 70$ \\
\hline HAM-2013 & $76-78$ & 5.8 & 0.33 & -16.0 & $62.50 \pm 0.60$ & $3920 \pm 80$ \\
\hline HAM-2014 & $78-80$ & 5.8 & 0.33 & -16.4 & $64.72 \pm 0.60$ & $3630 \pm 70$ \\
\hline HAM-2015 & $80-82$ & 5.7 & 0.26 & -17.0 & $63.37 \pm 0.60$ & $3790 \pm 80$ \\
\hline HAM-2016 & $82-84$ & 5.7 & 0.26 & -16.4 & $66.00 \pm 0.60$ & $3480 \pm 70$ \\
\hline HAM-2017 & $84-86$ & 5.7 & 0.29 & -15.8 & $62.46 \pm 0.59$ & $3930 \pm 80$ \\
\hline HAM-2018 & $86-88$ & 5.8 & 0.31 & -16.9 & $65.92 \pm 0.60$ & $3480 \pm 70$ \\
\hline HAM-2019 & $88-90$ & 5.8 & 0.22 & -17.1 & $66.74 \pm 0.61$ & $3380 \pm 70$ \\
\hline HAM-2020 & $90-92$ & 5.8 & 0.32 & -16.0 & $67.69 \pm 0.61$ & $3280 \pm 70$ \\
\hline HAM-2021 & $92-94$ & 5.8 & 0.29 & -16.7 & $67.25 \pm 0.61$ & $3320 \pm 70$ \\
\hline HAM-2022 & 94-96 & 5.8 & 0.24 & -17.3 & $67.28 \pm 0.61$ & $3310 \pm 70$ \\
\hline HAM-2023 & $96-98$ & 5.8 & 0.28 & -17.7 & $65.62 \pm 0.60$ & $3500 \pm 70$ \\
\hline HAM-2024 & $98-100$ & 5.8 & 0.25 & -17.9 & $65.74 \pm 0.60$ & $3480 \pm 70$ \\
\hline HAM-2025 & $100-102$ & 5.8 & 0.33 & -17.7 & $66.69 \pm 0.60$ & $3370 \pm 70$ \\
\hline HAM-2026 & $102-104$ & 5.8 & 0.24 & -17.6 & $64.73 \pm 0.59$ & $3610 \pm 70$ \\
\hline HAM-2027 & $104-106$ & 5.8 & 0.23 & -17.5 & $67.97 \pm 0.61$ & $3220 \pm 70$ \\
\hline HAM-2028 & $106-108$ & 5.8 & 0.27 & -18.1 & $65.98 \pm 0.60$ & $3450 \pm 70$ \\
\hline HAM-2029 & $108-110$ & 5.8 & 0.22 & -18.1 & $67.34 \pm 0.61$ & $3290 \pm 70$ \\
\hline HAM-2030 & $110-112$ & 5.8 & 0.30 & -17.1 & $71.70 \pm 0.62$ & $2800 \pm 70$ \\
\hline HAM-2031 & $112-114$ & 5.8 & 0.24 & -17.5 & $67.06 \pm 0.60$ & $3330 \pm 70$ \\
\hline HAM-2032 & $114-116$ & 5.8 & 0.25 & -17.3 & $66.88 \pm 0.60$ & $3360 \pm 70$ \\
\hline HAM-2033 & $116-118$ & 5.8 & 0.28 & -17.4 & $66.53 \pm 0.61$ & $3400 \pm 70$ \\
\hline HAM-2034 & $118-120$ & 6.0 & 0.19 & -18.1 & $67.31 \pm 0.61$ & $3290 \pm 70$ \\
\hline HAM-2035 & $120-122$ & 5.8 & 0.28 & -17.2 & $65.50 \pm 0.60$ & $3530 \pm 70$ \\
\hline HAM-2036 & $122-124$ & 6.4 & 0.24 & -17.5 & $66.30 \pm 0.60$ & $3420 \pm 70$ \\
\hline HAM-2037 & $124-126$ & 6.8 & 0.20 & -16.9 & $65.49 \pm 0.60$ & $3530 \pm 70$ \\
\hline
\end{tabular}


TABLE 12. Patancheru, Profile R (Continued)

\begin{tabular}{lcccccc}
\hline Lab code & Depth $(\mathrm{cm})$ & $\mathrm{pH}$ & $\mathrm{C}$ & $\delta^{13} \mathrm{C}$ & ${ }^{14} \mathrm{C}$ activity & ${ }^{14} \mathrm{C}$ age \\
\hline HAM-2038 & $126-128$ & 6.7 & 0.19 & -17.2 & $65.03 \pm 0.60$ & $3580 \pm 70$ \\
HAM-2039 & $128-130$ & 7.1 & 0.17 & -16.1 & $59.79 \pm 0.61$ & $4280 \pm 80$ \\
HAM-2040 & $130-132$ & 7.0 & 0.23 & -15.7 & $58.14 \pm 0.57$ & $4510 \pm 80$ \\
HAM-2041 & $132-134$ & 7.2 & 0.24 & -15.2 & $57.10 \pm 0.56$ & $4660 \pm 80$ \\
HAM-2042 & $134-136$ & 6.6 & 0.23 & -17.2 & $65.68 \pm 0.60$ & $3500 \pm 70$ \\
HAM-2043 & $136-138$ & 6.6 & 0.19 & -16.9 & $64.81 \pm 0.59$ & $3620 \pm 70$ \\
HAM-2044 & $138-140$ & 6.9 & 0.25 & -15.3 & $60.28 \pm 0.58$ & $4220 \pm 80$ \\
HAM-2045 & $140-142$ & 7.1 & 0.24 & -15.0 & $59.68 \pm 0.57$ & $4150 \pm 80$ \\
HAM-2046 & $142-144$ & 7.1 & 0.23 & -15.4 & $60.60 \pm 0.58$ & $4180 \pm 80$ \\
HAM-2047 & $144-146$ & 7.2 & 0.27 & -13.1 & $55.91 \pm 0.56$ & $4670 \pm 80$ \\
HAM-2048 & $146-148$ & 7.1 & 0.33 & -12.8 & $54.04 \pm 0.55$ & $4940 \pm 80$ \\
HAM-2049 & $148-150$ & 7.2 & 0.41 & -10.8 & $46.80 \pm 0.61$ & $6100 \pm 110$ \\
HAM-2050 & $150-152$ & 6.9 & 0.29 & -12.1 & $50.57 \pm 0.54$ & $5480 \pm 90$ \\
HAM-2051 & $152-154$ & 7.1 & 0.40 & -11.3 & $50.22 \pm 0.54$ & $5530 \pm 90$ \\
HAM-2052 & $154-156$ & 7.0 & 0.34 & -11.8 & $49.44 \pm 0.56$ & $5660 \pm 90$ \\
HAM-2053 & $156-158$ & 7.1 & 0.38 & -10.9 & $46.77 \pm 0.53$ & $6100 \pm 90$ \\
\hline
\end{tabular}

*No isotope correction

Bulk density: $1.5-1.56 \mathrm{~g} \mathrm{~cm}^{-3}$, according to VERMA (1990), cited in Singer (1993).

\section{Los BAÑos}

\section{Profile-Related Data}

Location (longitude, latitude): $14^{\circ} 10^{\prime} \mathrm{N}, 121^{\circ} 16^{\prime} \mathrm{E}$

Location (country, next city or village): Philippines, Los Baños, Luzon, IRRI

Soil order and type (USDA classification): Aeric Tropaquept, fine, mixed, nonacid/noncalcareous, isohyperthermic

Soil order and type (local classification): Maahas clay series

Parent material:

Mean annual temperature:

Mainly volcanic tuffa

Annual rainfall:

$26^{\circ} \mathrm{C}$

Vegetation and land use:

Site description:

$2150 \mathrm{~mm}$

Rice (former coconut)

$21 \mathrm{~m}$ asl

The Aeric Tropaquept on the IRRI farm at Los Baños was chosen, because a site of this series has also been used for decomposition experiments with ${ }^{14} \mathrm{C}$-labeled rice straw (Martin 1985; Neue and Scharpenseel 1987). The soil was sampled after harvest, before puddling. In earlier geological periods the area was below the sea level of the nearby laguna.

Date of sampling:

1983

Date of measurement:

1985

Collector:

Becker-Heidmann

Submitter:

Becker-Heidmann

Submitter's comment:

Because of intensive rice cultivation, the Los Baños profile has a low organic $\mathrm{C}$ content and, consequently, a high ${ }^{14} \mathrm{C}$ activity level in the topsoil. The ${ }^{14} \mathrm{C}$ activity is uniform down to $8 \mathrm{~cm}$, with a value of $121 \mathrm{pMC}$; below, in the main rooty zone, there is a maximum. After puddling, the ${ }^{14} \mathrm{C}$ activity should be the same everywhere within the Apg. The ${ }^{14} \mathrm{C}$ activity of the carbon input to the soil has been decreasing from one season to the next because of the continuing decrease of the ${ }^{14} \mathrm{C}$ activity of the atmosphere since the atomic bomb 
test ban treaty. Therefore, at the time of sampling, after harvest and before puddling, we expected in the main rooty zone between 10 and $14 \mathrm{~cm}$ a lower ${ }^{14} \mathrm{C}$ activity than elsewhere in the Apg. But the uppermost $8 \mathrm{~cm}$ showed a lower ${ }^{14} \mathrm{C}$ activity. This may be interpreted as a rapid translocation of the freshly decomposed material to the surface. This interpretation is supported by the results of decomposition experiments with ${ }^{14} \mathrm{C}$-labeled rice straw in the same soil (Becker-Heidmann et al. 1985).

The uniform value of $\delta^{13} \mathrm{C}$ in the entire $\mathrm{Ap} 1 \mathrm{~g}$ horizon indicates regular homogenization by puddling, whereas the Ap2g seems to be rarely puddled. Below the Apg horizon, the ${ }^{14} \mathrm{C}$ activity decreases to $\mathrm{ca} .100$ $\mathrm{pMC}$; the ${ }^{14} \mathrm{C}$ increases. The portion of soluble, percolating organic substances is obviously too low to cause a considerable rejuvenation in the subsoil. The steep slope of the ${ }^{14} \mathrm{C}$ activity and the $\delta^{13} \mathrm{C}$ curve at the plow pan, together with the low $\mathrm{C}$ content and maximum clay concentration at this layer, indicates a strong fixation of highly metabolized and aged humic substances to the clay. The percolating younger material passes through to the bottom, as can be concluded from both the $\delta^{13} \mathrm{C}$ inflection and the high ${ }^{14} \mathrm{C}$ activity values in the whole basal soil.

Spikes of high-concentration bomb carbon can be found in the lower part of the ${ }^{14} \mathrm{C}$ activity depth distribution curve. These are located exactly at the transition layers of adjacent horizons, where there are wetter conditions than in the middle of the horizons, because drainage is impeded by texture changes. Thus, percolating organic matter cannot be bound permanently to clay in these layers, which would raise the ${ }^{14} \mathrm{C}$ age. Another explanation could be that fine roots would concentrate in these layers and, because of their high turnover rate, permanently introduce modern C. However, the amount of fresh root material necessary for such a shift in ${ }^{14} \mathrm{C}$ activity would also significantly alter $\delta^{13} \mathrm{C}$, which has not been observed.

Literature references:

Becker-Heidmann and Scharpenseel (1989); Scharpenseel and Becker-Heidmann (1993); Neue et al. (1990); Scharpenseel and Becker-Heidmann (1994b); Martin (1985); Scharpenseel, Pfeiffer and Becker-Heidmann (1995a, 1995b)

\section{Description of the Profile at Los Baños:}

\begin{tabular}{|c|c|c|}
\hline Depth $(\mathrm{cm})$ & Horizon & Description \\
\hline $0-14$ & Ap1g & Black (10YR 2/1), fine roots \\
\hline $14-22$ & Ap2g & \\
\hline $22-36$ & IIA3 & $\begin{array}{l}\text { Black (10YR } 2 / 1) \text {, yellowish brown (10YR } 5 / 6) \text { mottles, larger aggre- } \\
\text { gates with reddish brown coating (Fe), little fine roots }\end{array}$ \\
\hline $36-50$ & IIB1 & Yellowish brown, black tongues from IIA3 \\
\hline $50-76$ & IIB2 & Yellowish brown, green-blue tongues from IIB2g \\
\hline $76-90$ & IIB2 $g$ & Green-blue \\
\hline $90+$ & IIIR & Green-blue, large brown mottles (tuffa) \\
\hline
\end{tabular}

TABLE 13. Los Baños

\begin{tabular}{lcccccc}
\hline Lab code & Depth $(\mathrm{cm})$ & $\mathrm{pH}$ & $\mathrm{C}$ & $\delta^{13} \mathrm{C}$ & ${ }^{14} \mathrm{C}$ activity & ${ }^{14} \mathrm{C}$ age \\
\hline HAM-2147 & $0-2$ & 6.0 & 2.02 & -22.8 & $121.80 \pm 0.73$ & Modern \\
HAM-2148 & $2-4$ & 5.8 & 2.26 & -22.9 & $121.80 \pm 0.72$ & Modern \\
HAM-2149 & $4-6$ & 5.6 & 2.08 & -23.0 & $121.40 \pm 0.72$ & Modern \\
HAM-2150 & $6-8$ & 5.7 & 1.90 & -22.8 & $121.35 \pm 0.77$ & Modern \\
HAM-2151 & $8-10$ & 5.8 & 1.89 & -22.9 & $123.65 \pm 0.76$ & Modern \\
HAM-2152 & $10-12$ & 6.1 & 1.82 & -22.7 & $124.60 \pm 0.73$ & Modern \\
HAM-2153 & $12-14$ & 6.1 & 1.65 & -22.8 & $123.20 \pm 0.73$ & Modern \\
HAM-2154 & $14-16$ & 6.1 & 2.10 & -22.3 & $121.36 \pm 0.72$ & Modern \\
HAM-2155 & $16-18$ & 6.4 & 1.65 & -22.0 & $121.36 \pm 0.76$ & Modern \\
HAM-2156 & $18-20$ & 6.4 & 1.36 & -21.5 & $118.48 \pm 0.71$ & Modern
\end{tabular}


TABLE 13. Los Baños (Continued)

\begin{tabular}{lcccccc}
\hline Lab code & Depth(cm) & $\mathrm{pH}$ & $\mathrm{C}$ & $\delta^{13} \mathrm{C}$ & ${ }^{14} \mathrm{C}$ activity & ${ }^{14} \mathrm{C}$ age \\
\hline HAM-2157 & $20-22$ & 6.2 & 1.41 & -20.8 & $116.08 \pm 0.70$ & Modern \\
HAM-2158 & $22-24$ & 6.3 & 0.99 & -19.0 & $109.27 \pm 0.67$ & Modern \\
HAM-2159 & $24-26$ & 6.3 & 0.93 & -17.7 & $106.52 \pm 0.67$ & Modern \\
HAM-2160 & $26-28$ & 6.5 & 0.80 & -16.8 & $104.76 \pm 0.66$ & Modern \\
HAM-2161 & $28-30$ & 6.4 & 0.63 & -16.1 & $101.61 \pm 0.65$ & Modern \\
HAM-2162 & $30-32$ & 6.7 & 0.64 & -16.1 & $101.11 \pm 0.65$ & Modern \\
HAM-2163 & $32-34$ & 6.5 & 0.70 & -16.1 & $102.45 \pm 0.65$ & Modern \\
HAM-2164 & $34-36$ & 6.6 & 0.52 & -16.2 & $111.29 \pm 0.68$ & Modern \\
HAM-2165 & $36-38$ & 6.6 & 0.55 & -15.9 & $106.19 \pm 0.66$ & Modern \\
HAM-2166 & $38-40$ & 6.5 & 0.51 & -15.8 & $101.62 \pm 0.65$ & Modern \\
HAM-2167 & $40-42$ & 6.5 & 0.45 & -15.7 & $103.74 \pm 0.66$ & Modern \\
HAM-2168 & $42-44$ & 6.5 & 0.45 & -15.8 & $108.65 \pm 0.67$ & Modern \\
HAM-2169 & $44-46$ & 6.5 & 0.42 & -15.7 & $104.21 \pm 0.68$ & Modern \\
HAM-2170 & $46-48$ & 6.5 & 0.36 & -15.8 & $102.45 \pm 0.65$ & Modern \\
HAM-2171 & $48-50$ & 6.5 & 0.35 & -15.9 & $119.38 \pm 0.72$ & Modern \\
HAM-2172 & $50-52$ & 6.5 & 0.30 & -16.0 & $104.08 \pm 0.66$ & Modern \\
HAM-2173 & $52-54$ & 6.5 & 0.30 & -16.0 & $102.87 \pm 0.72$ & Modern \\
HAM-2174 & $54-56$ & 6.4 & 0.29 & -16.0 & $102.52 \pm 0.65$ & Modern \\
HAM-2175 & $56-58$ & 6.6 & 0.28 & -16.0 & $109.69 \pm 0.68$ & Modern \\
HAM-2176 & $58-60$ & 6.5 & 0.26 & -16.2 & $101.43 \pm 0.65$ & Modern \\
HAM-2177 & $60-62$ & 6.5 & 0.24 & -16.1 & $101.51 \pm 0.65$ & Modern \\
HAM-2178 & $62-64$ & 6.3 & 0.24 & -16.3 & $100.03 \pm 0.64$ & Modern \\
HAM-2179 & $64-66$ & 6.6 & 0.23 & -16.4 & $102.30 \pm 0.66$ & Modern \\
HAM-2180 & $66-68$ & 6.5 & 0.23 & -16.6 & $102.01 \pm 0.65$ & Modern \\
HAM-2181 & $68-70$ & 6.4 & 0.21 & -16.5 & $103.46 \pm 0.65$ & Modern \\
HAM-2182 & $70-72$ & 6.4 & 0.22 & -16.6 & $99.78 \pm 0.64$ & $150 \pm 50$ \\
HAM-2183 & $72-74$ & 6.5 & 0.22 & -16.8 & $103.00 \pm 0.65$ & Modern \\
HAM-2184 & $74-76$ & 6.4 & 0.19 & -16.9 & $99.89 \pm 0.69$ & $140 \pm 60$ \\
HAM-2185 & $76-78$ & 6.5 & 0.19 & -17.3 & $107.81 \pm 0.67$ & Modern \\
HAM-2186 & $78-80$ & 6.7 & 0.20 & -17.3 & $109.08 \pm 0.86$ & Modern \\
HAM-2187 & $80-82$ & 6.7 & 0.20 & -17.0 & $102.14 \pm 0.72$ & Modern \\
\hline
\end{tabular}

Bulk density: (Martin 1985) wet (not directly applicable for our data, as our soil was sampled during dry season

\begin{tabular}{cc}
\hline Depth $(\mathrm{cm})$ & Bulk density $\left(\mathrm{g} \mathrm{cm}^{-3}\right)$ \\
\hline $0-5$ & 1.08 \\
$5-10$ & 1.13 \\
$10-15$ & 1.22 \\
$15-20$ & 1.37 \\
\hline
\end{tabular}

PangIL

\section{Profile-Related Data}

Location (longitude, latitude):

Location (country, next city or village):

Soil order and type (FAO classification):

Soil order and type (USDA classification): $14^{\circ} 24^{\prime} \mathrm{N}, 121^{\circ} 28^{\prime} \mathrm{E}$

Philippines, Pangil, Luzon

Eutric Gleysol

Aeric Tropaquept, fine, kaolinitic, nonacid/noncalcareous, isohyperthermic 
Soil order and type (local classification):

Parent material:

Mean annual temperature:

Bay clay series

Alluvium (unspecified)

Annual rainfall:

$26^{\circ} \mathrm{C}$

Vegetation and land use:

$2150 \mathrm{~mm}$

Site description: $<5 \mathrm{~m}$ asl, flat

Rice (since $>10 \mathrm{yr}$ )

The soil is on the site of an experimental station of IRRI. It has been used for lowland rice, but should be prepared for an upland rice experiment shortly after the sampling. The soil is known to suffer from phosphorusdeficiency. The profile was located within a plot that has never been fertilized.

Date of sampling:

1983

Date of measurement:

$1986 / 1987$

Collector:

Becker-Heidmann

Submitter:

Submitter's comment:

The $\delta^{13} \mathrm{C}$ value is $c a .-22 \%$ in Apg, a value that has been found in several other tropical rice soils (see below). The increase of $\delta^{13} \mathrm{C}$ with depth due to decomposition is rather small. The ${ }^{14} \mathrm{C}$ activity is the same as the ${ }^{14} \mathrm{C}$ activity of the atmosphere in the topsoil. An inhibited decomposition in topsoil can be inferred from the slight increase of $\delta^{13} \mathrm{C}$ combined with the high $\mathrm{C}$ content. This interpretation fits with the known phosphorus deficiency and the low $\mathrm{pH}$ in this alluvial soil. It is also known that the farmers had not continuously grown rice in this field because of the low productivity. The ${ }^{14} \mathrm{C}$ activity decreases very steeply below the plow pan, indicating again the low decomposition but also a low infiltration rate of fresh organic matter, i.e., an effective hardpan.

Lab comment:

Literature references:

Becker-Heidmann and Scharpenseel (1992a); Scharpenseel and Becker-Heidmann (1989)

\section{Description of Profile at Pangil}

Depth $(\mathrm{cm})$ Horizon Description

\begin{tabular}{rll}
\hline $0-16$ & Apg & Dark yellowish brown (10YR 3/4) \\
$16-20$ & ABcn & Manganese concretions \\
$20-60$ & Bg1 & Grayish brown (10YR 3/2), red mottles (increasing to the bottom) \\
$60+$ & Bg2 & Lateral water seepage and groundwater table at 70+1
\end{tabular}

TABLE 14. Pangi

\begin{tabular}{lcccccc}
\hline Lab code & Depth $(\mathrm{cm})$ & $\mathrm{pH}$ & $\mathrm{C}$ & $\delta^{13} \mathrm{C}$ & ${ }^{14} \mathrm{C}$ activity & ${ }^{14} \mathrm{C}$ age \\
\hline HAM-2365 & $0-2$ & 4.8 & 2.45 & -21.8 & $122.06 \pm 0.84$ & Modern \\
HAM-2366 & $2-4$ & 4.6 & 2.33 & -22.0 & $122.46 \pm 0.87$ & Modern \\
HAM-2367 & $4-6$ & 4.6 & 2.35 & -22.1 & $122.92 \pm 0.84$ & Modern \\
HAM-2368 & $6-8$ & 4.7 & 2.32 & -22.2 & $122.12 \pm 0.84$ & Modern \\
HAM-2369 & $8-10$ & 4.8 & 2.20 & -22.1 & $124.56 \pm 0.85$ & Modern \\
HAM-2370 & $10-12$ & 4.9 & 1.88 & -22.3 & $121.16 \pm 0.93$ & Modern \\
HAM-2371 & $12-14$ & 5.2 & 1.62 & -22.5 & $119.73 \pm 0.88$ & Modern \\
HAM-2372 & $14-16$ & 5.7 & 1.37 & -22.3 & $113.03 \pm 0.80$ & Modern \\
HAM-2373 & $16-18$ & 6.1 & 0.90 & -21.4 & $104.53 \pm 0.76$ & Modern \\
HAM-2374 & $18-20$ & 6.0 & 0.62 & -20.8 & $96.84 \pm 0.73$ & $330 \pm 60$ \\
HAM-2375 & $20-22$ & 6.0 & 0.48 & -21.2 & $90.24 \pm 0.70$ & $890 \pm 60$ \\
HAM-2376 & $22-24$ & -- & -- & -- & -- & - \\
HAM-2377 & $24-26$ & 6.0 & 0.49 & -20.1 & $89.33 \pm 0.70$ & $980 \pm 60$ \\
HAM-2378 & $26-28$ & 6.1 & 0.47 & -21.4 & $86,33 \pm 0.65$ & $1240 \pm 60$
\end{tabular}


TABLE 14. Pangi (Continued)

\begin{tabular}{lcccccc}
\hline Lab code & Depth $(\mathrm{cm})$ & $\mathrm{pH}$ & $\mathrm{C}$ & $\delta^{13} \mathrm{C}$ & ${ }^{14} \mathrm{C}$ activity & ${ }^{14} \mathrm{C}$ age \\
\hline HAM-2379 & $28-30$ & 6.0 & 0.47 & -21.2 & $83.75 \pm 0.61$ & $1490 \pm 50$ \\
HAM-2380 & $30-32$ & 6.0 & 0.41 & -20.7 & $84,85 \pm 0.73$ & $1390 \pm 70$ \\
HAM-2381 & $32-34$ & 6.1 & 0.42 & -21.4 & $83.78 \pm 0.60$ & $1480 \pm 50$ \\
HAM-2382 & $34-36$ & 6.0 & 0.36 & -20.5 & $83.94 \pm 0.65$ & $1480 \pm 60$ \\
HAM-2383 & $36-38$ & 6.1 & 0.38 & -20.5 & $82.35 \pm 0.60$ & $1630 \pm 50$ \\
HAM-2384 & $38-40$ & 6.1 & 0.37 & -20.7 & $82.63 \pm 0.67$ & $1600 \pm 70$ \\
HAM-2385 & $40-42$ & 5.9 & 0.32 & -20.5 & $83.43 \pm 0.68$ & $1530 \pm 70$ \\
HAM-2386 & $42-44$ & 5.9 & 0.35 & -20.1 & $83.75 \pm 0.68$ & $1500 \pm 70$ \\
HAM-2387 & $44-46$ & 6.1 & 0.48 & -19.7 & $91.87 \pm 0.71$ & $770 \pm 60$ \\
HAM-2388 & $46-48$ & 6.0 & 0.30 & -19.8 & $83.59 \pm 0.67$ & $1520 \pm 70$ \\
HAM-2389 & $48-50$ & 6.2 & 0.37 & -20.8 & $82.38 \pm 0.68$ & $1630 \pm 70$ \\
HAM-2390 & $50-52$ & 6.1 & 0.33 & -21.1 & $84.19 \pm 0.66$ & $1440 \pm 60$ \\
HAM-2391 & $52-54$ & 6.3 & 0.35 & -21.1 & $81.28 \pm 0.65$ & $1730 \pm 60$ \\
HAM-2392 & $54-56$ & 5.8 & 0.31 & -21.5 & $79.75 \pm 0.64$ & $1870 \pm 70$ \\
HAM-2393 & $56-58$ & 5.9 & 0.31 & -21.5 & $78.95 \pm 0.66$ & $1960 \pm 70$ \\
HAM-2394 & $58-60$ & 6.1 & 0.32 & -21.9 & $80.57 \pm 0.65$ & $1790 \pm 70$ \\
HAM-2395 & $60-62$ & 5.9 & 0.28 & -21.9 & $80.68 \pm 0.64$ & $1770 \pm 60$ \\
HAM-2396 & $62-64$ & 5.7 & 0.32 & -21.0 & $77.60 \pm 0.63$ & $2100 \pm 70$ \\
HAM-2397 & $64-66$ & 5.8 & 0.32 & -21.7 & $80.65 \pm 0.64$ & $1780 \pm 60$ \\
HAM-2398 & $66-68$ & 5.6 & 0.32 & -22.9 & $76.58 \pm 0.77$ & $2180 \pm 80$ \\
HAM-2399 & $68-70$ & 5.6 & 0.31 & -21.8 & $50.92 \pm 0.69$ & $5470 \pm 60$ \\
\hline
\end{tabular}

Pao

\section{Profile-Related Data}

Location (longitude, latitude):

$16^{\circ} \mathrm{N}, 120^{\circ} 16^{\prime} \mathrm{E}$

Location (country, next city or village): $\quad$ Philippines, Pao, Pangasinan, Luzon

Soil order and type (USDA classification): Aeric Tropaquept, fine silty over clayey, mixed, noncalcareous, isohyperthermic

Soil order and type (local classification): San Manuel clay loam series

Mean annual temperature:

$28^{\circ} \mathrm{C}$

Annual rainfall:

Vegetation and land use:

$2250 \mathrm{~mm}$

Rice (rainy season) / Mungbeans (dry season, planned)

Site description:

Date of sampling: -.

1983

Date of measurement:

1990

Collector:

Submitter:

Submitter's comment:

Becker-Heidmann

Becker-Heidmann

The organic carbon content is low, ca. $1 \%$ in the Apg and $<0.5 \%$ in the subsoil. The $\delta^{13} \mathrm{C}$ value as well as the increase of ${ }^{13} \mathrm{C}$ from the surface through the B1 is comparatively high. This might be due to methane formation. The ${ }^{14} \mathrm{C}$ activity in the Ap1g is only slight lower than the ${ }^{14} \mathrm{C}$ activity of the atmosphere, indicating a low decomposition activity. The comparatively high activity in the subsoil, its very slow decrease, and especially the single very high value corresponding to a low $\delta^{13} \mathrm{C}$ at the $\mathrm{B} 1-\mathrm{B} 2 \mathrm{cn}$ boundary, indicate a high percolation rate.

Lab comment: 
Literature references:

Becker-Heidmann (1990); Scharpenseel and BeckerHeidmann (1994a); Scharpenseel and Becker-

Heidmann (1994b)

Description of the Profile at Pao:

\begin{tabular}{lll} 
Depth $(\mathrm{cm})$ & Horizon & Description \\
\hline $0-10$ & Ap1g & Puddled zone, 8-10 “traffic pan" \\
$10-19$ & Ap2g & Seldomly puddled zone \\
$19-50$ & B1 & \\
$50-83$ & B2cn & Iron and manganese concretions \\
$83+$ & B2g & Grayish blue
\end{tabular}

TABLE 15. Pao

\begin{tabular}{|c|c|c|c|c|c|c|}
\hline Lab code & Depth $(\mathrm{cm})$ & $\mathrm{pH}$ & $\mathrm{C}$ & $\delta^{13} \mathrm{C}$ & ${ }^{14} \mathrm{C}$ activity & ${ }^{14} \mathrm{C}$ age* \\
\hline HAM-2330 & $0-2$ & 7.0 & 0.98 & -18.7 & $114.02 \pm 0.80$ & Modern \\
\hline & & & 0.97 & -18.2 & & \\
\hline HAM-2332 & 4 & & 0.99 & -18.4 & $114.80 \pm 0.81$ & lodern \\
\hline & 6 & 7.1 & 1.02 & -17.9 & $117.49 \pm 0.82$ & Modern \\
\hline HAM-2334 & $8-10$ & 7.0 & 0.95 & -17.7 & $114.26 \pm 0.62$ & Modern \\
\hline HAM-2335 & 10 & & 0.93 & -17.2 & $112.39 \pm 0.62$ & Modern \\
\hline & & 7.2 & & -16.2 & $111.48 \pm 0.03$ & Modern \\
\hline & & 6.9 & 0.71 & -14.9 & \pm 0.77 & Modern \\
\hline 2320 & & 6.9 & 0. & -15.2 & & $130 \pm 60$ \\
\hline & & 6.9 & & -14.5 & $97.86 \pm 0.75$ & \pm 60 \\
\hline & & 6.9 & 0.49 & -14.3 & 79 & $0 \pm 70$ \\
\hline & & 6.9 & 0.52 & -14.3 & & $0 \pm 70$ \\
\hline & & & 0.49 & -13.7 & & $410 \pm 70$ \\
\hline & & 6.9 & 0 & & & \\
\hline & & & 0.4 & -15.3 & & \pm 50 \\
\hline & & 6.9 & 0.3 & -14.6 & 73 & \pm 70 \\
\hline & & 6 & 0.42 & -14.0 & & 70 \\
\hline & & & 0.34 & -14.5 & & \pm 70 \\
\hline & & 6 & 0.36 & -14.0 & & $0 \pm 70$ \\
\hline 0 & & 7 & 0.36 & -13.5 & & \pm 50 \\
\hline & & & 0.32 & -13.5 & & \pm 70 \\
\hline & & 6.9 & 0.29 & -15.1 & 128 & Modern \\
\hline-2352 & & & 0.28 & -15.7 & & $670 \pm 70$ \\
\hline 2252 & & 6 & 0.29 & -13.8 & $4 \pm 0.72$ & $10 \pm 70$ \\
\hline & & & 0.26 & -14.3 & $9 \pm 0.73$ & \pm 70 \\
\hline $101-2 J J$ & & & 0.25 & -14.9 & & \\
\hline AM-2356 & & 6.5 & 0.24 & -14.6 & & $930 \pm 70$ \\
\hline & & 6 & & -14.6 & & $840 \pm 50$ \\
\hline AM-2358 & & 6.6 & & -14.4 & & $000+70$ \\
\hline HAM-2359 & $71-74$ & 6.8 & & -14.6 & 73 & $1110 \pm 70$ \\
\hline HAM-2360 & & 6.5 & & & $7.78 \pm 0.75$ & $350 \pm 70$ \\
\hline UA 2261 & & 6.5 & 0.27 & -14.8 & $0.10 \pm 0.55$ & $1000 \pm 50$ \\
\hline 2562 & & 6.3 & 0.29 & -14.6 & \pm 0.72 & $1050 \pm 70$ \\
\hline HA & & 7.3 & 0.31 & -14.6 & & $1120 \pm 70$ \\
\hline HAM-2364 & $86-88$ & 6.5 & 0.33 & -14.6 & $87.64 \pm 0.71$ & $1230 \pm ?$ \\
\hline
\end{tabular}

${ }^{*} \delta^{13} \mathrm{C}$ correction applied, as the high $\delta^{13} \mathrm{C}$ here results not from $\mathrm{C}_{4}$ plants but from methane production. 


\section{BUgALLON}

\section{Profile-Related Data}

Location (longitude, latitude): $15^{\circ} 55^{\prime} \mathrm{N}, 120^{\circ} 15^{\prime} \mathrm{E}$

Location (country, next city or village): Philippines, Bugallon, Pangasinan, Luzon

Soil order and type (USDA classification): Haplic Hydraquent, fine loamy to sandy, mixed, non-

Parent material: calcareous, isohyperthermic

Mean annual temperature: Alluvium

Annual rainfall: $28^{\circ} \mathrm{C}$ $2250 \mathrm{~mm}$

Vegetation and land use:

Site description:

Grasses; rice until $3 \mathrm{yr}$ before sampling

The soil profile at Bugallon is located near Pao, ca. $700 \mathrm{~m}$ west of the road from Tarlac to Dagupan City. The area belongs to the western part of the Agno River basin between the foothills of the Zambales Mountains in the west and the floodplains of the Agno River. The surface relief is the result of alternate sedimentation and erosion processes during the development of the river beds. The soil has been studied by Haupenthal et al. (1979) with regard to $\mathrm{Zn}$ deficiency and by Scharpenseel et al. (1981) with regard to $\mathrm{Cr}$ - and Ni-toxicity and the possible interaction with the $\mathrm{Zn}$-deficiency. A P deficiency has also been noticed. The soil has developed from ultrabasic, ophiolitic and basic alluvium of the nearby Zambales hills.

Date of sampling: 1983

Date of measurement:

1987

Collector:

Submitter:

Submitter's comment:

The Bugallon soil was sampled only down to $30 \mathrm{~cm}$, because artesian groundwater rapidly filled the profile. The carbon content decreases steeply at the plow pan, from $2 \%$ in the topsoil to $0.5 \%$ in the subsoil.

The $\delta^{13} \mathrm{C}$ value increases very steeply from a usual value of $-22 \%$ at the surface to $-14 \%$ in $\mathrm{B} 2 \mathrm{~g}$. Here we have an indication of a decomposition producing mainly methane.

Lab comment:

Literature references:

Becker-Heidmann (1990); Scharpenseel and BeckerHeidmann (1994b)

\section{Description of the Profile at Bugallon:}

\begin{tabular}{cll} 
Depth $(\mathrm{cm})$ & Horizon & Description \\
\hline $0-12$ & Ap & Black, rooty to very rooty, silty clay \\
$12-45+$ & B2g & $\begin{array}{l}\text { Olive brown to black, little rooty, clay loam } \\
\text { Groundwater table from artesian activity }\end{array}$
\end{tabular}

TABLE 16. Bugallon

\begin{tabular}{lcccccc}
\hline Lab code & Depth $(\mathrm{cm})$ & $\mathrm{pH}$ & $\mathrm{C}$ & $\delta^{13} \mathrm{C}$ & ${ }^{14} \mathrm{C}$ activity & ${ }^{14} \mathrm{C}$ age \\
\hline HAM-2315 & $0-2$ & 7.7 & 2.17 & -22.0 & $116.19 \pm 0.63$ & Modern \\
HAM-2316 & $2-4$ & 7.4 & 2.22 & -21.2 & $116.69 \pm 0.64$ & Modern \\
HAM-2317 & $4-6$ & 7.1 & 2.18 & -21.6 & $109.07 \pm 0.71$ & Modern \\
HAM-2318 & $6-8$ & 7.4 & 1.96 & -21.4 & $114.76 \pm 0.62$ & Modern \\
HAM-2319 & $8-10$ & 7.5 & 1.89 & -20.9 & $111.01 \pm 0.66$ & Modern \\
HAM-2320 & $10-12$ & 7.3 & 1.39 & -20.4 & $109.62 \pm 0.60$ & Modern \\
HAM-2321 & $12-14$ & 7.6 & 1.04 & -17.4 & $104.88 \pm 0.61$ & Modern \\
HAM-2322 & $14-16$ & 7.4 & 0.79 & -14.5 & $100.96 \pm 0.65$ & Modern
\end{tabular}


TABLE 16. Bugallon (Continued)

\begin{tabular}{lcccccr}
\hline Lab code & Depth $(\mathrm{cm})$ & $\mathrm{pH}$ & $\mathrm{C}$ & $\delta^{13} \mathrm{C}$ & ${ }^{14} \mathrm{C}$ activity & \multicolumn{1}{c}{${ }^{14} \mathrm{C}$ age } \\
\hline HAM-2323 & $16-18$ & 6.7 & 0.68 & -13.5 & $95.71 \pm 0.81$ & $540 \pm 70$ \\
HAM-2324 & $18-20$ & 6.9 & 0.62 & -13.4 & $95.43 \pm 0.67$ & $560 \pm 60$ \\
HAM-2325 & $20-22$ & 7.2 & 0.46 & -13.3 & $95.10 \pm 0.56$ & $595 \pm 45$ \\
HAM-2326 & $22-24$ & 6.7 & 0.75 & -13.8 & $67.74 \pm 0.80$ & $3310 \pm 90$ \\
HAM-2327 & $24-26$ & 6.9 & 0.76 & -13.9 & $65.75 \pm 0.79$ & $3550 \pm 90$ \\
HAM-2328 & $26-28$ & 6.5 & 0.71 & -13.5 & $66.54 \pm 0.78$ & $3460 \pm 90$ \\
HAM-2329 & $28-30$ & 6.6 & 0.52 & -13.4 & $65.18 \pm 0.78$ & $3630 \pm 90$ \\
\hline
\end{tabular}

\section{Tiaong, Profile L}

\section{Profile-Related Data}

Location (longitude, latitude): $13^{\circ} 59^{\prime} \mathrm{N}, 121^{\circ} 21^{\prime} \mathrm{E}$

Location (country, next city or village): Philippines, Tiaong, Luzon

Soil order and type (USDA classification): Haplic Hydraquent, coarse silty, mixed, calcareous,

Parent material: isohyperthermic

Mean annual temperature:

Volcanic ash alluvium from Mt. Banahaw

Annual rainfall: $26^{\circ} \mathrm{C}$

Vegetation and land use:

Site description:

$2150 \mathrm{~mm}$

Rice (grasses at sampling time)

43 asl

The profile is the lowest one of a toposequence, investigated earlier by Scharpenseel (1978) and Scharpenseel et al. (1981). It is similar to the Bugallon profile, but contains much more plant debris and organic matter. It contains a large number of different species of snails, some of them down to $60 \mathrm{~cm}$ depth. Big roots, probably from bamboo, were found in 50 to $60 \mathrm{~cm}$ depth (not within the sampling area). Artesian water filled the profile up to $30 \mathrm{~cm}$ depth, when the sampling reached $60 \mathrm{~cm}$. The local problems with rice cultivation result from a pronounced $\mathrm{Zn}$ deficiency. At the time of sampling the soil was fallow and covered by grasses.

Date of sampling:

1983

Date of measurement: $\quad 1990$

Collector:

Submitter:

Becker-Heidmann

Becker-Heidmann

Submitter's comment:

The lower profile of the Tiaong toposequence is characterized by a high $\mathrm{pH}$ of 7.5 to 8 throughout the profile and the highest organic carbon content of all studied Philippine rice soils. It contains also a great deal of carbonate, $>3 \%$ in the topsoil and even $2 \%$ in most parts of the subsoil. The $\delta^{13} \mathrm{C}$ value of the organic matter increases with depth within the Apg, as expected, and decreases below the plow pan in the subsoil. As the $\delta^{13} \mathrm{C}$ value of the carbonate lies between the $\delta^{13} \mathrm{C}$ value of primary carbonates and the $\delta^{13} \mathrm{C}$ of fresh $\mathrm{C}_{3}$ plant material, it is obvious that organic and inorganic carbon have exchanged in this profile. The parallel depth distribution curves of organic and inorganic $\delta^{13} \mathrm{C}$ suggest that this exchange is permanently occurring.

Lab comment:

Literature references:

--

Becker-Heidmann (1990); Scharpenseel and BeckerHeidmann 1994b

\section{Description of Profile $L$ at Tiaong}

\begin{tabular}{cll} 
Depth $(\mathrm{cm})$ & Horizon & Description \\
\hline $0-16$ & Ap1g & Dark brown, very rooty, fibers of bamboo \\
$16-22$ & Ap2g & Dark brown, very rooty
\end{tabular}


22-58 B21g Bluish green, rooty

58-64+ B22g Green blue, snails

TABLE 17. Tiaong, Profile L

\begin{tabular}{|c|c|c|c|c|c|c|c|c|c|c|}
\hline Lab code & Depth $(\mathrm{cm})$ & $\mathrm{pH}$ & $\mathrm{C}_{\text {org }}$ & $\delta^{13} C_{\text {org }}$ & ${ }^{14} \mathrm{C}_{\text {org }}$ act. & ${ }^{14} \mathrm{C}_{\text {org }}$ age & $\mathrm{C}_{\text {in }}$ & $\delta^{13} C_{\text {in }}$ & ${ }^{4} C_{\text {in }}$ act. & ${ }^{4} C_{\text {in }}$ age \\
\hline $\mathrm{HA}$ & $0-2$ & 7.9 & 9.27 & -21.6 & $113.69 \pm 0.81$ & Modern & 3.29 & -8.7 & .76 & $180 \pm 60$ \\
\hline HA & & 7.9 & 8.58 & -222 & $.32 \pm 0.80$ & & 3.41 & -8.2 & 0.75 & \pm 60 \\
\hline HAM-2190 & $4-6$ & 8.0 & 8.89 & -21.7 & 112.5 & Modern & 3.57 & -7.1 & $91.85 \pm 0.73$ & $680 \pm 70$ \\
\hline HAM-2191 & $6-$ & 8.1 & 8.52 & -18.7 & $118.42 \pm$ & Modern & 3.23 & -4.8 & 0.55 & $765 \pm 50$ \\
\hline HAM-2192 & $8-10$ & 8.2 & 7.98 & -17.9 & $115.16 \pm 0.81$ & Modern & 3.14 & -5.3 & $97.42 \pm 0.75$ & $210 \pm 60$ \\
\hline HAM-2193 & $10-12$ & 8.0 & 7.74 & -18.8 & 116 & $\mathrm{rn}$ & 3.28 & -5.1 & $115.66 \pm 0.82$ & Modern \\
\hline HAM-2194 & $12-14$ & 7.7 & 6.47 & -17.2 & $118.42 \pm 0.82$ & & 3.37 & -7.5 & 0.76 & Modern \\
\hline HAM-2195 & & 8.0 & 6.65 & -17.8 & $94.20 \pm 0.74$ & 70 & 3.47 & -5.7 & 0.74 & $\neq 60$ \\
\hline HAl & $16-18$ & 7.8 & 6.13 & -17.4 & $118.32 \pm 0.64$ & ern & 3.87 & -5.6 & .57 & \\
\hline HA & & 7.8 & 5.94 & -16.6 & $112.87 \pm 0.80$ & m & 3.25 & -5.4 & 0.75 & \\
\hline HA & & 8.0 & 5.53 & -19.0 & & & 2.93 & -5.9 & 0.72 & 12 \\
\hline HA & 2 & 8.0 & 2.01 & -19.0 & $104.13 \pm 0.77$ & & 2.65 & -6.5 & 90 & 80 \\
\hline HAl & $24-26$ & 7.6 & 4.34 & -18.9 & $03 \pm 0.58$ & & 2.32 & -7.5 & 0.53 & 50 \\
\hline $\mathrm{HA}$ & $26-28$ & 7.7 & 4.08 & -19.3 & & & 2.54 & -7.5 & .70 & 70 \\
\hline 202 & $28-30$ & 7.6 & 3.37 & -19.4 & & & 2.75 & -7.1 & .70 & 70 \\
\hline $\mathrm{HA}$ & $30-32$ & 7.5 & 4.88 & -19.8 & 74 & & 2.38 & -8.4 & .69 & 163 \\
\hline HAl & 32 & 7.6 & 4.00 & -18.9 & 91.1 & & 2.88 & -8.5 & .70 & 19 \\
\hline 205 & $34-36$ & 7.5 & 3.31 & -19.1 & 91.4 & & 3.01 & -8.3 & .70 & \\
\hline-2206 & $36-38$ & 7.5 & 3.60 & -19.1 & 89.1 & 10 & 2.71 & -8.4 & .70 & 17 \\
\hline 207 & $38-40$ & 7.4 & 4.17 & -19.4 & 93.4 & & 2.42 & -8.9 & .70 & 16 \\
\hline 2208 & -42 & 7.5 & 3.50 & -19.6 & .73 & & 2.85 & -8.5 & .57 & 60 \\
\hline HAM-2209 & $42-44$ & 7.4 & 4.30 & -19.2 & $92.16 \pm \ldots$ & & 2.60 & -10.9 & 78 & 60 \\
\hline 2210 & -46 & 7.6 & 2.93 & -20.3 & $90.53 \pm 0.72$ & \pm 70 & 2.40 & -9.2 & .69 & 70 \\
\hline 11 & & 7.5 & 2.85 & -20.4 & $91.52 \pm 0.73$ & $790 \pm 70$ & 2.71 & -9.2 & 0.69 & 171 \\
\hline 12 & & 7.5 & 2.95 & -20.2 & $93.12 \pm 0.73$ & \pm 70 & 2.60 & -8.9 & 0.60 & 60 \\
\hline $\mathrm{H}$ & & 7.4 & 2.58 & -20.8 & $118.09 \pm 0.78$ & Modern & 2.67 & -9.4 & $81.23 \pm 0.69$ & 167 \\
\hline HAl & -54 & 7.5 & 2.37 & -20.5 & $93.71 \pm 0.73$ & $590 \pm 70$ & 2.28 & - & $80.39 \pm 0.59$ & $1750 \pm 60$ \\
\hline & & 7.6 & 2.05 & -22.0 & $95.17 \pm 0.73$ & $450 \pm 60$ & 2.16 & -8.7 & $80.26 \pm 0.68$ & $1770 \pm 70$ \\
\hline 2216 & -58 & 7.3 & 1.79 & -21.8 & $101.11 \pm 0.76$ & Modern & 1.79 & -9.1 & $86.55 \pm 0.54$ & $1160 \pm 50$ \\
\hline HAM-2217 & & 7.4 & 0.93 & -22.4 & & \pm 70 & 1.53 & -9.3 & $82.44 \pm 0.69$ & $1550 \pm 70$ \\
\hline HAM-2218 & $60-62$ & 8.0 & 1.19 & -23.3 & $89.49 \pm 0.71$ & $920 \pm 70$ & 0.75 & -9.8 & $81.63 \pm 0.69$ & $1630 \pm 70$ \\
\hline HAM-2219 & & 7.3 & 0.72 & -24.7 & -- & -- & 0.46 & -10.6 & -- & -- \\
\hline HAM-2220 & $64-66$ & 7.3 & 1.02 & -25.2 & $99.90 \pm 0.58$ & $5 \pm 50$ & 0.27 & -14.7 & $85.81 \pm 0.71$ & $1230 \pm 70$ \\
\hline
\end{tabular}

\section{Tiaong, Profile H}

\section{Profile-Related Data}

Location (longitude, latitude):

\section{$13^{\circ} 59^{\prime} \mathrm{N}, 121^{\circ} 21^{\prime} \mathrm{E}$}

Location (country, next city or village): Philippines, Tiaong, Luzon

Soil order and type (USDA classification): Typic Haplaquoll, fine silty, mixed, noncalcareous, isohyperthermic

Parent material:

Mean annual temperature:

Volcanic ash from nearby Mt. Banahaw

Annual rainfall:

Vegetation and land use:

Site description:

$26^{\circ} \mathrm{C}$

$2150 \mathrm{~mm}$

Banana, coconut

$45 \mathrm{~m}$ asl

This profile was located at the top of the toposequence at Tiaong, $c a .200 \mathrm{~m}$ apart from Profile $\mathrm{L}$ in a small banana and coconut grove, and was characterized by an epiaquic water regime.

Date of sampling:

1983 
Date of measurement:

Collector:

Submitter:

Submitter's comment:

The soil had not been plowed; thus, its $\mathrm{A} 11$ horizon shows a maximum of the ${ }^{14} \mathrm{C}$ activity between 6 and 8 $\mathrm{cm}$ below surface, in the main rooty zone, and a constant decrease below that. In the $\mathrm{A} 12$ horizon the ${ }^{14} \mathrm{C}$ activity varies ca. $110 \mathrm{pMC}$, and in the $\mathrm{A} 12$ and $\mathrm{B} 2 \mathrm{~g}$ horizons there are only a few layers where ${ }^{14} \mathrm{C}$ activity is $<100 \mathrm{pMC}$. This means that a continuous admixture of fresh organic matter had occurred throughout the whole profile. Because of the wet conditions combined with nonintensive cultivation, an accumulation of organic matter is probable. With decreasing clay content, $\delta^{13} \mathrm{C}$ decreases with depth and ${ }^{14} \mathrm{C}$ activity increases slightly. At the transition zone between the A12 and the water-saturated B2g horizon, the redox potential seasonally changes with the groundwater level, as proven by a strong enrichment of $\mathrm{Mn}$ in the layers of the ${ }^{14} \mathrm{C}$ activity peaks. These conditions prevent fixation and aging of organic substances and lead to the "modern" ${ }^{14} \mathrm{C}$ activity peak.

The fact that organic substances with high ${ }^{14} \mathrm{C}$ activity ("bomb radiocarbon", i.e., built after the beginning of thermonuclear testing) are found in the subsoil can be interpreted as intact bioturbation within the total profile depth of this Mollisol. Measurements of the ${ }^{14} \mathrm{C}$ activity of earthworms from a Mollisol showing the "bomb carbon" in worms of all depths, support this hypothesis (Scharpenseel et al. 1986). Recent carbon can be introduced also in clay-organic complexes of all depths in this clayey and silty soil. As a comparison, the age of the total organic matter of a Mollisol from Inner Mongolia in China (Becker-Heidmann, Liu and Scharpenseel 1988) increases with $69.3 \mathrm{yr} \mathrm{cm}^{-1}$ depth, which corresponds to a very slow soil development via an extended pararendzina phase. Here $\delta^{13} \mathrm{C}$ does not deviate from the value of fresh plant material throughout the profile, indicating early protection against further degradation. The reason for the slow soil development is most probably the climatic regime, with temperatures of $-20^{\circ} \mathrm{C}$ and $<25 \mathrm{~mm}$ rainfall in January and $c a .18^{\circ} \mathrm{C}$ and between 25 and $50 \mathrm{~mm}$ rainfall in June, rather than the influence of the geological conditions.

Literature references:

\section{Description of the Profile at Tiaong $(\mathrm{H})$ :}

\begin{tabular}{rll} 
Depth $(\mathrm{cm})$ & Horizon & Description \\
\hline $0-20$ & A11 & Black, rooty, big earthworms \\
$20-36$ & A12 & Slightly rooty \\
$36-60$ & B2 & Grayish brown, reddish brown mottles, no roots \\
60 & & Groundwater table
\end{tabular}

TABLE 18. Tiaong $(\mathrm{H})$

\begin{tabular}{lcccccc}
\hline Lab code & Depth $(\mathrm{cm})$ & $\mathrm{pH}$ & $\mathrm{C}$ & $\delta^{13} \mathrm{C}$ & ${ }^{14} \mathrm{C}$ activity & ${ }^{14} \mathrm{C}$ age \\
\hline HAM-2222 & $0-2$ & 6.4 & 3.74 & -20.2 & $118.43 \pm 0.82$ & Modern \\
HAM-2223 & $2-4$ & 6.3 & 3.98 & -20.1 & $119.23 \pm 0.82$ & Modern \\
HAM-2224 & $4-6$ & 6.7 & 3.47 & -19.7 & $118.32 \pm 0.82$ & Modern \\
HAM-2225 & $6-8$ & 6.9 & 3.44 & -19.5 & $120.96 \pm 0.83$ & Modern \\
HAM-2226 & $8-10$ & 7.0 & 2.71 & -19.3 & $115.75 \pm 0.81$ & Modern \\
HAM-2227 & $10-12$ & 6.9 & 2.67 & -19.3 & $116.54 \pm 0.82$ & Modern \\
HAM-2228 & $12-14$ & 7.2 & 2.42 & -18.7 & $111.57 \pm 0.79$ & Modern \\
HAM-2229 & $14-16$ & 7.2 & 2.36 & -18.5 & $110.72 \pm 0.79$ & Modern \\
HAM-2230 & $16-18$ & 7.1 & 1.86 & -17.8 & $107.92 \pm 0.78$ & Modern \\
HAM-2231 & $18-20$ & 7.2 & 1.75 & -16.5 & $103.15 \pm 0.76$ & Modern
\end{tabular}

Becker-Heidmann and Scharpenseel (1989); Scharpenseel and Becker-Heidmann (1992b); Scharpenseel et al. (1989) 
TABLE 18. Tiaong $(\mathrm{H})$ (Continued)

\begin{tabular}{lccccrc}
\hline Lab code & Depth $(\mathrm{cm})$ & $\mathrm{pH}$ & $\mathrm{C}$ & ${ }^{13} \mathrm{C}$ & \multicolumn{1}{c}{${ }^{14} \mathrm{C}$ activity } & ${ }^{14} \mathrm{C}$ age \\
\hline HAM-2232 & $20-22$ & 7.0 & 1.53 & -16.2 & $102.72 \pm 0.75$ & Modern \\
HAM-2233 & $22-24$ & 7.0 & 1.31 & -16.3 & $101.55 \pm 0.75$ & Modern \\
HAM-2234 & $24-26$ & 7.1 & 1.27 & -15.8 & $98.37 \pm 0.74$ & $280 \pm 60$ \\
HAM-2235 & $26-28$ & 7.0 & 1.16 & -15.8 & $101.18 \pm 0.75$ & Modern \\
HAM-2236 & $28-30$ & 7.1 & 0.92 & -16.0 & $101.16 \pm 0.75$ & Modern \\
HAM-2237 & $30-32$ & 7.1 & 0.80 & -16.5 & $100.03 \pm 0.74$ & Modern \\
HAM-2238 & $32-34$ & 7.0 & 0.63 & -16.8 & $98.97 \pm 0.74$ & $220 \pm 60$ \\
HAM-2239 & $34-36$ & 6.9 & 0.59 & -17.3 & $100.63 \pm 0.75$ & Modern \\
HAM-2240 & $36-38$ & 6.9 & 0.50 & -17.8 & $107.59 \pm 0.77$ & Modern \\
HAM-2241 & $38-40$ & 6.9 & 0.47 & -18.1 & $107.08 \pm 0.77$ & Modern \\
HAM-2242 & $40-42$ & 7.0 & 0.36 & -18.3 & $100.06 \pm 0.74$ & Modern \\
HAM-2243 & $42-44$ & 7.1 & 0.41 & -18.4 & $99.74 \pm 0.74$ & $130 \pm 60$ \\
HAM-2244 & $44-46$ & 6.9 & 0.40 & -18.7 & $96.80 \pm 0.75$ & $360 \pm 60$ \\
HAM-2245 & $46-48$ & 6.8 & 0.31 & -15.9 & $103.86 \pm 0.76$ & Modern \\
HAM-2246 & $48-50$ & 6.9 & 0.31 & -16.8 & $100.24 \pm 0.74$ & Modern \\
HAM-2247 & $50-52$ & 6.9 & 0.30 & -19.6 & $102.84 \pm 0.77$ & Modern \\
HAM-2248 & $52-54$ & 6.6 & 0.30 & -18.9 & $101.55 \pm 0.79$ & Modern \\
\hline
\end{tabular}

SAN Dionisio, Profile T

\section{Profile-Related Data}

Location (longitude, latitude):

$11^{\circ} 32^{\prime} \mathrm{N}, 123^{\circ} 07^{\prime} \mathrm{E}$

Location (country, next city or village): $\quad$ Philippines, San Dionisio, Panay

Soil order and type (USDA classification): Typic Paleudult, coarse loamy over clayey, mixed,

Parent material:

Mean annual temperature: oxidic, acid, isohyperthermic

Annual rainfall:

Vegetation and land use:

Colluvium above estuarine clayey alluvium

$26^{\circ} \mathrm{C}$

$3000 \mathrm{~mm}$

Site description:

Rice, presently fallow

The profile was located in the northernmost part of the island Panay, near the village San Dionisio. The area is part of a former river delta system. At San Dionisio, the former sediment contains pure kaolonite, which is now being mined for pottery. It is covered by colluvium of $300 \mathrm{~cm}$ depth from a nearby mountain with a high iron content. Therefore, the soil suffers from iron toxicity and is not very suitable for rice production. Some of the stones found below $100 \mathrm{~cm}$ have been dated to $>16,000 \mathrm{yr}$ (V. Toquillo, personal communication).

Date of sampling:

1983

Date of measurement:

1985

Collector:

Submitter:

Becker-Heidmann, V. P. Singh

Becker-Heidmann

Submitter's comment:

This profile has developed under tidal influence in a river estuary, where sedimentation and mangrove growing caused high contents of clay as well as organic matter. Microbial reduction of sulfates from seawater and anaerobic decomposition of the organic matter led to a decrease of the $\mathrm{pH}$ value and subsequently, a reduction of the minerals of the sediment to kaolinite. Later, the iron-rich colluvium of the nearby hill covered this acid sulfate soil. The present situation is characterized by the reduction of $\mathrm{Fe}_{3}+$ to $\mathrm{Fe}_{2}+$ under low $\mathrm{pH}$ and, simultaneously, the oxidation of $\mathrm{SOM}$ with a release of $\mathrm{Fe}_{2}+$ in toxic concentrations under the anaerobic conditions of rice cultivation. 
An unexpectedly high value of $\delta^{13} \mathrm{C}$ in and its decrease with depth may be explained by the field lying fallow and subsequent covering by tropical grasses, among which are probably some $\mathrm{C}_{4}$ species. This is supported by the ${ }^{14} \mathrm{C}$ activity being nearly as high as the atmospheric level and indicating a continued high $\mathrm{C}$ input to the soil. Organic matter in the puddling zone is dominated by bomb carbon of the sampling year concentration (ca. $120 \mathrm{pMC}$ ), which indicates a high turnover rate. The high ${ }^{14} \mathrm{C}$ activity in the topsoil, together with the low carbon content, show also that the decomposition and humification is very low in this soil. Especially in the layers where $\delta^{13} \mathrm{C}$ is constant, no biological activity was detected by IRRI's Dept. of Microbiology (V. P. Singh, personal communication 1983). The low biological activity is probably caused by the large percentage of kaolinite with a low cation exchange capacity responsible for a weak dispersion of the soil.

The soil below $\mathrm{B} 1$ shows a constant $\delta^{13} \mathrm{C}$ value with a continuous and rapid decrease in ${ }^{14} \mathrm{C}$ activity and a correspondingly increasing ${ }^{14} \mathrm{C}$ age. The steep increase of the ${ }^{14} \mathrm{C}$ age up to $\mathrm{ca} .3000 \mathrm{yr}$ already at $80 \mathrm{~cm}$ depth can be explained by the high clay content of $c a .50 \%$. We conclude that organic matter in the lower part of the profile is well protected against further metabolization or admixture of young material.

Literature references:

Becker-Heidmann and Scharpenseel (1989)

Description of the Profile at San Dionisio:

Depth $(\mathrm{cm})$ Horizon Description

\begin{tabular}{lll}
\hline $0-20$ & Apg & Brown-gray, very rooty \\
$20-36$ & B1 & Light brown-gray, reddish mottles (iron), no roots \\
$36-70$ & B2 & Light reddish brown \\
$70+$ & B2 & Light reddish brown, intense red mottles, alluvium rich in iron
\end{tabular}

TABLE 19. San Dionisio, Profile T

\begin{tabular}{lcccccc}
\hline Lab code & Depth(cm) & pH & \multicolumn{1}{c}{ C } & \multicolumn{1}{c}{$\delta^{13} \mathrm{C}$} & \multicolumn{1}{c}{14 C activity } & \multicolumn{1}{c}{${ }^{14}$ age } \\
\hline HAM-2249 & $0-2$ & 3.9 & 1.48 & -17.4 & $117.58 \pm 0.82$ & Modern \\
HAM-2250 & $2-4$ & 3.7 & 1.07 & -18.3 & $121.42 \pm 0.84$ & Modern \\
HAM-2251 & $4-6$ & 3.7 & 0.99 & -19.1 & $116.57 \pm 0.82$ & Modern \\
HAM-2252 & $6-8$ & 3.7 & 0.92 & -18.8 & $121.56 \pm 0.84$ & Modern \\
HAM-2253 & $8-10$ & 3.7 & 0.83 & -18.5 & $120.42 \pm 0.83$ & Modern \\
HAM-2254 & $10-12$ & 3.8 & 0.74 & -18.8 & $120.00 \pm 0.83$ & Modern \\
HAM-2255 & $12-14$ & 3.6 & 0.69 & -18.8 & $109.65 \pm 0.80$ & Modern \\
HAM-2256 & $14-16$ & 4.2 & 0.60 & -19.3 & $105.48 \pm 0.83$ & Modern \\
HAM-2257 & $16-18$ & 3.5 & 0.60 & -19.2 & $102.21 \pm 0.76$ & Modern \\
HAM-2258 & $18-20$ & 4.0 & 0.57 & -19.4 & $100.03 \pm 0.74$ & Modern \\
HAM-2259 & $20-22$ & 4.0 & 0.58 & -19.6 & $99.00 \pm 0.75$ & $170 \pm 60$ \\
HAM-2260 & $22-24$ & 3.4 & 0.48 & -20.0 & $99.99 \pm 0.74$ & $80 \pm 60$ \\
HAM-2261 & $24-26$ & 3.7 & 0.50 & -20.9 & $106.95 \pm 0.60$ & Modern \\
HAM-2262 & $26-28$ & 3.5 & 0.53 & -22.1 & $93.50 \pm 0.72$ & $590 \pm 60$ \\
HAM-2263 & $28-30$ & 3.5 & 0.49 & -23.0 & $94.03 \pm 0.78$ & $530 \pm 70$ \\
HAM-2264 & $30-32$ & 3.5 & 0.48 & -22.9 & $102.15 \pm 0.74$ & Modern \\
HAM-2265 & $32-34$ & 3.6 & 0.49 & -22.7 & $92.09 \pm 0.66$ & $700 \pm 60$ \\
HAM-2266 & $34-36$ & 3.7 & 0.47 & -22.7 & $93.17 \pm 0.62$ & $600 \pm 50$ \\
HAM-2267 & $36-38$ & 3.5 & 0.48 & -22.3 & $90.09 \pm 0.60$ & $880 \pm 50$ \\
HAM-2268 & $38-40$ & 3.7 & 0.47 & -22.3 & $88.38 \pm 0.60$ & $1040 \pm 50$ \\
HAM-2269 & $40-42$ & 4.2 & 0.55 & -22.3 & $89.53 \pm 0.60$ & $930 \pm 50$ \\
HAM-2270 & $42-44$ & 3.8 & 0.55 & -22.4 & $87.70 \pm 0.60$ & $1100 \pm 50$ \\
HAM-2271 & $44-46$ & 3.6 & 0.55 & -22.7 & $80.96 \pm 0.57$ & $1730 \pm 60$ \\
HAM-2272 & $46-48$ & 3.8 & 0.50 & -22.3 & $83.36 \pm 0.67$ & $1510 \pm 70$ \\
HAM-2273 & $48-50$ & 3.7 & 0.54 & -22.3 & $83.44 \pm 0.61$ & $1500 \pm 60$ \\
HAM-2274 & $50-60$ & 3.6 & 0.56 & -22.3 & $81.21 \pm 0.57$ & $1720 \pm 60$ \\
& & & & & &
\end{tabular}


TABLE 19. San Dionisio, Profile T (Continued)

\begin{tabular}{lcccccc}
\hline Lab code & Depth(cm) & $\mathrm{pH}$ & $\mathrm{C}$ & $\delta^{13} \mathrm{C}$ & ${ }^{14} \mathrm{C}$ activity & ${ }^{14} \mathrm{C}$ age \\
\hline HAM-2275 & $60-70$ & 3.6 & 0.57 & -22.1 & $73.41 \pm 0.59$ & $2530 \pm 60$ \\
HAM-2276 & $70-80$ & 3.9 & 0.54 & -22.0 & $70.23 \pm 0.53$ & $2890 \pm 60$ \\
HAM-2277 & $80-90$ & 3.6 & 0.57 & -20.2 & $60.81 \pm 0.50$ & $4070 \pm 70$ \\
HAM-2278 & $90-110$ & 3.4 & 0.63 & -19.3 & $52.87 \pm 0.47$ & $5210 \pm 70$ \\
HAM-2279 & $110-130$ & 3.7 & 0.53 & -20.4 & $60.76 \pm 0.58$ & $4080 \pm 80$ \\
HAM-2280 & $130-150$ & 3.9 & 0.58 & -21.0 & $47.38 \pm 0.53$ & $6070 \pm 90$ \\
HAM-2281 & $150-170$ & 3.7 & 0.44 & -17.7 & $41.56 \pm 0.60$ & $7170 \pm 120$ \\
HAM-2282 & $170-190$ & 3.7 & 0.59 & -16.1 & $31.02 \pm 0.39$ & $9550 \pm 100$ \\
HAM-2283 & $190-210$ & 3.9 & 0.51 & -15.6 & $30.29 \pm 0.39$ & $9750 \pm 100$ \\
HAM-2284 & $210-240$ & 3.3 & 0.50 & -15.7 & $45.63 \pm 0.52$ & $6450 \pm 90$ \\
HAM-2285 & $240-270$ & 3.2 & 0.73 & -12.6 & $19.58 \pm 0.35$ & $13,300 \pm 150$ \\
HAM-2286 & $270-285$ & 3.3 & 0.31 & -15.3 & $23.69 \pm 0.52$ & $11,730 \pm 180$ \\
HAM-2287 & $285-300$ & 3.1 & 0.28 & -14.9 & $25.19 \pm 0.37$ & $11,240 \pm 120$ \\
HAM-2288 & $300-315$ & 3.9 & 0.46 & -13.7 & $25.86 \pm 0.55$ & $11,050 \pm 170$ \\
HAM-2289 & $315-330$ & 3.4 & 0.66 & -13.4 & $13.53 \pm 0.35$ & $16,260 \pm 210$ \\
HAM-2290 & $330-340$ & 3.7 & 1.85 & -12.2 & $9.70 \pm 0.39$ & $18,950 \pm 330$ \\
HAM-2291 & $340-350$ & 3.7 & 1.31 & -12.6 & $8.67 \pm 0.32$ & $19,850 \pm 300$ \\
HAM-2292 & $350-360$ & 3.6 & 1.21 & -12.4 & $8.03 \pm 0.32$ & $20,460 \pm 320$ \\
HAM-2293 & $360-370$ & 4.0 & 0.80 & -12.9 & $8.14 \pm 0.32$ & $20,350 \pm 310$ \\
HAM-2294 & $370-380$ & 3.3 & 0.64 & -14.1 & $8.77 \pm 0.32$ & $19,730 \pm 290$ \\
HAM-2295 & $380-390$ & 3.9 & 0.74 & -14.1 & $7.17 \pm 0.39$ & $21,350 \pm 430$ \\
HAM-2296 & $390-410$ & 3.6 & 5.06 & -15.2 & $2.88 \pm 0.37$ & $28,660 \pm 1040$ \\
HAM-2297 & $410-430$ & 3.8 & 4.98 & -14.4 & $4.06 \pm 0.38$ & $25,910 \pm 750$ \\
HAM-2298 & $430-450$ & 4.2 & 2.07 & -14.4 & $4.46 \pm 0.38$ & $25,150 \pm 680$ \\
HAM-2299 & $450-470$ & 3.8 & 4.14 & -14.6 & $4.08 \pm 0.49$ & $25,870 \pm 960$ \\
HAM-2300 & $470-490$ & 3.1 & 1.89 & -13.6 & $4.91 \pm 0.38$ & $24,400 \pm 620$ \\
HAM-2301 & $490-510$ & 3.8 & 1.36 & -15.8 & $4.10 \pm 0.38$ & $25,810 \pm 740$ \\
\hline
\end{tabular}

\section{NAMTOU HSIEN}

\section{Profile-Related Data}

Location (longitude, latitude):

\section{$24^{\circ} 08^{\prime} \mathrm{N}, 120^{\circ} 41^{\prime} \mathrm{E}$}

Location (country, next city or village): Republic of China, Namtou Hsien near Taichung

Soil order and type (USDA classification): Typic Fluvaquent, fine to moderate

Parent material:

Sandstone and shales alluvium

Mean annual temperature:

$22^{\circ} \mathrm{C}$

Annual rainfall:

Vegetation and land use:

$2250 \mathrm{~mm}$

Rice

Site description:

The soil developed in a relatively new deposit above an old soil, which also was used for rice.

Date of sampling:

1984

Date of measurement:

1989

Collector:

Becker-Heidmann, H.Y. Guo

Submitter:

Becker-Heidmann, H.Y. Guo

Submitter's comment:

The $\delta^{13} \mathrm{C}$ value was low in the Ap1, $-26 \%$, corresponding to fresh plant material from rice. We observed a steep increase within the Ap2; in the subsoil the $\delta^{13} \mathrm{C}$ value was $c a .-16 \%$ PDB. As the fossil soil below was also used for growing rice and not for, e.g., sugar cane, this increase can easily be explained by decomposi- 
tion processes involving larger amounts of $\mathrm{CH}_{4}$. The ${ }^{14} \mathrm{C}$ activity value of only $108 \mathrm{pMC}$ in the Ap1 strongly supports the hypothesis of a high decomposition rate. The curve in the subsoil shows the normal pattern.

Literature references:

Becker-Heidmann (1990); Scharpenseel and BeckerHeidmann (1994b)

Description of the Profile at Namtou Hsien:

Depth (cm) Horizon Description

\begin{tabular}{rll}
\hline $0-10$ & Ap1 & Very dark gray brown, clay loam \\
$10-20$ & Ap2 & Dark gray brown \\
$20-40$ & IIfAp & Silty clay \\
$40-50$ & IIAB & Gray brown \\
$50-70$ & IIB21 & Gray brown \\
$70+$ & IIB22 & Gray brown
\end{tabular}

TABLE 20. Namtsou Hsien

\begin{tabular}{lcccccc}
\hline Lab code & Depth $(\mathrm{cm})$ & $\mathrm{pH}$ & $\mathrm{C}$ & $\delta^{13} \mathrm{C}$ & ${ }^{14} \mathrm{C}$ activity & ${ }^{14} \mathrm{C}$ age \\
\hline HAM-2661 & $0-2$ & 4.5 & 1.60 & -26.3 & $107.10 \pm 0.79$ & Modern \\
HAM-2662 & $2-4$ & 4.6 & 1.64 & -25.9 & $108.09 \pm 0.80$ & Modern \\
HAM-2663 & $4-6$ & 4.8 & 1.50 & -25.4 & $106.69 \pm 0.80$ & Modern \\
HAM-2664 & $6-8$ & 4.9 & 1.57 & -25.9 & $105.77 \pm 0.78$ & Modern \\
HAM-2665 & $8-10$ & 5.4 & 1.47 & -25.8 & $107.50 \pm 0.61$ & Modern \\
HAM-2666 & $10-12$ & 5.8 & 1.51 & -25.0 & $106.58 \pm 0.79$ & Modern \\
HAM-2667 & $12-14$ & 5.9 & 1.32 & -25.6 & $107.00 \pm 0.79$ & Modern \\
HAM-2668 & $14-16$ & 6.1 & 1.05 & -24.0 & $104.81 \pm 0.79$ & Modern \\
HAM-2669 & $16-18$ & 6.0 & 0.85 & -21.7 & $100.11 \pm 0.76$ & Modern \\
HAM-2670 & $18-20$ & 6.2 & 0.87 & -20.6 & $98.93 \pm 0.58$ & $160 \pm 50$ \\
HAM-2671 & $20-22$ & 6.3 & 0.86 & -19.3 & $94.12 \pm 0.75$ & $580 \pm 70$ \\
HAM-2672 & $22-24$ & 6.3 & 0.84 & -17.0 & $92.71 \pm 0.74$ & $740 \pm 70$ \\
HAM-2673 & $24-26$ & 6.3 & 0.89 & -16.1 & $93.00 \pm 0.74$ & $730 \pm 70$ \\
HAM-2674 & $26-28$ & 6.3 & 0.88 & -16.1 & $91.49 \pm 0.73$ & $860 \pm 70$ \\
HAM-2675 & $28-30$ & 6.1 & 1.02 & -16.4 & $93.90 \pm 0.57$ & $645 \pm 50$ \\
HAM-2676 & $30-32$ & 6.2 & 1.07 & -15.7 & $93.96 \pm 0.75$ & $650 \pm 70$ \\
HAM-2677 & $32-34$ & 6.1 & 1.11 & -15.6 & $91.02 \pm 0.73$ & $910 \pm 70$ \\
HAM-2678 & $34-36$ & 6.2 & 1.32 & -15.7 & $90.78 \pm 0.74$ & $930 \pm 70$ \\
HAM-2679 & $36-38$ & 6.1 & 1.48 & -15.7 & $92.82 \pm 0.75$ & $750 \pm 70$ \\
HAM-2680 & $38-40$ & 6.2 & 1.72 & -14.8 & $89.78 \pm 0.73$ & $1030 \pm 70$ \\
HAM-2681 & $40-42$ & 6.2 & 1.75 & -15.2 & $90.24 \pm 0.56$ & $985 \pm 50$ \\
HAM-2682 & $42-44$ & 6.1 & 1.20 & -14.8 & $89.62 \pm 0.73$ & $1050 \pm 70$ \\
HAM-2683 & $44-46$ & 6.1 & 1.17 & -14.7 & $86.96 \pm 0.73$ & $1290 \pm 70$ \\
HAM-2684 & $46-48$ & 6.1 & 1.13 & -14.8 & $86.16 \pm 0.72$ & $1360 \pm 70$ \\
HAM-2685 & $48-50$ & 6.2 & 1.04 & -15.3 & $86.32 \pm 0.54$ & $1340 \pm 50$ \\
HAM-2686 & $50-60$ & 6.2 & 0.97 & -15.4 & $84.71 \pm 0.71$ & $1490 \pm 70$ \\
HAM-2687 & $60-70$ & 6.1 & 0.78 & -16.0 & $81.63 \pm 0.84$ & $1780 \pm 80$ \\
HAM-2688 & $70-80$ & 5.9 & 0.73 & -16.1 & $81.36 \pm 0.95$ & $1800 \pm 100$ \\
\hline
\end{tabular}

Pingtung

\section{Profile-Related Data}

Location (longitude, latitude): $\quad 22^{\circ} 40^{\prime} \mathrm{N}, 120^{\circ} 29^{\prime} \mathrm{E}$ 
Location (country, next city or village):

Republic of China, experimental station of Pingtung near Kaohsiung

Soil order and type (USDA classification): Typic Fluvaquent

Soil order and type (local classification):

Parent material:

Mean annual temperature:

Slate alluvium

Annual rainfall:

Vegetation and land use:

Site description:

Date of sampling:

$24^{\circ} \mathrm{C}$

$2500 \mathrm{~mm}$

$2 x$ rice $+1 \times$ beans per year for $20 y r$

Date of measurement:

1984

1989

Collector:

Submitter:

Becker-Heidmann, Guo

Becker-Heidmann, Guo

Submitter's comment:

The carbon content is rather low, with $1.2 \%$ near the surface and $<0.3 \%$ in the subsoil. The $\delta^{13} \mathrm{C}$ value shows the normal pattern of increasing with depth within the topsoil. Its shift is not as pronounced as in the Taichung soil. Therefore, methane production might be not as high as in the Taichung profile. The ${ }^{14} \mathrm{C}$ activity is generally comparable in value and curve to the Taichung soil. This indicates that the strength of decomposition is also comparable. A considerable difference in the depth distribution pattern can be observed at the boundaries of the horizons of the subsoil. High to extremely high ${ }^{14} \mathrm{C}$ activity shows that considerable amounts of fresh soluble organic substances have been percolating down through the profile. In spite of the low organic matter content of the soil and of losses by percolation, the productivity of this soil is rather high. In a soil fertility study of 130 soils of Kaohsiung-Pingtung region, Chang et al. (1983) showed that the morphological characteristics of the subsoil created by illuviation, deposition, reduction, oxidation, and groundwater behavior has no response to rice production and surface soil fertility.

Lab comment:

Literature references:

Becker-Heidmann and Scharpenseel (1992a)

Description of the Profile at Pingtung:

\begin{tabular}{cll} 
Depth $(\mathrm{cm})$ & Horizon & Description \\
\hline $0-16$ & Ap1 & Brown-gray, brown mottles, rooty \\
$16-26$ & Ap2 & Brown-gray to ochre, many reddish mottles \\
$26-44$ & B1 & Gray-brown, light brown mottles, silty \\
$44-80$ & B21 & Gray-brown to brown \\
$80+$ & B22 &
\end{tabular}

TABLE 21. Pingtung

\begin{tabular}{lccccrc}
\hline Lab code & Depth $(\mathrm{cm})$ & $\mathrm{pH}$ & $\mathrm{C}$ & $\delta^{13} \mathrm{C}$ & ${ }^{14} \mathrm{C}$ activity & ${ }^{14} \mathrm{C}$ age \\
\hline HAM-2689 & $0-2$ & 4.6 & 1.20 & -22.1 & $108.49 \pm 0.80$ & Modern \\
HAM-2690 & $2-4$ & 4.7 & 1.18 & -23.0 & $111.46 \pm 0.62$ & Modern \\
HAM-2691 & $4-6$ & 4.7 & 1.15 & -22.9 & $112.36 \pm 0.81$ & Modern \\
HAM-2692 & $6-8$ & 4.7 & 1.08 & -23.1 & $110.76 \pm 0.80$ & Modern \\
HAM-2693 & $8-10$ & 4.6 & 0.37 & -23.1 & $97.09 \pm 0.74$ & $270 \pm 60$ \\
HAM-2694 & $10-12$ & 4.8 & 0.87 & -22.6 & $105.98 \pm 0.78$ & Modern \\
HAM-2695 & $12-14$ & 5.1 & 1.00 & -22.5 & $106.69 \pm 0.78$ & Modern \\
HAM-2696 & $14-16$ & 5.4 & 0.83 & -22.5 & $102.46 \pm 0.77$ & Modern \\
HAM-2697 & $16-18$ & 5.5 & 0.75 & -21.9 & $66.60 \pm 0.66$ & $3320 \pm 80$ \\
HAM-2698 & $18-20$ & 5.8 & 0.59 & -21.3 & $91.36 \pm 0.74$ & $790 \pm 70$ \\
HAM-2699 & $20-22$ & 5.8 & 0.48 & -20.2 & $89.55 \pm 0.55$ & $965 \pm 50$
\end{tabular}


TABLE 21. Pingtung (Continued)

\begin{tabular}{lccccrc}
\hline Lab code & Depth $(\mathrm{cm})$ & $\mathrm{pH}$ & $\mathrm{C}$ & $\delta^{13} \mathrm{C}$ & ${ }^{14} \mathrm{C}$ activity & ${ }^{14} \mathrm{C}$ age \\
\hline HAM-2700 & $22-24$ & 6.3 & 0.36 & -19.5 & $87.89 \pm 0.72$ & $1130 \pm 70$ \\
HAM-2701 & $24-26$ & -- & 1.16 & -19.1 & $105.55 \pm 0.78$ & Modern \\
HAM-2702 & $26-28$ & 6.1 & 0.44 & -18.3 & $84.74 \pm 0.73$ & $1440 \pm 70$ \\
HAM-2703 & $28-30$ & 6.1 & 0.36 & -18.6 & $82.97 \pm 0.71$ & $1600 \pm 70$ \\
HAM-2704 & $30-32$ & 6.1 & 0.41 & -17.6 & $82.77 \pm 0.65$ & $1640 \pm 60$ \\
HAM-2705 & $32-34$ & 6.2 & 0.43 & -17.6 & $92.09 \pm 0.75$ & $780 \pm 70$ \\
HAM-2706 & $34-36$ & 6.2 & 0.44 & -17.2 & $100.39 \pm 1.26$ & Modern \\
HAM-2707 & $36-38$ & 6.1 & 0.45 & -17.3 & $84.71 \pm 0.54$ & $1460 \pm 50$ \\
HAM-2708 & $38-40$ & 6.1 & 0.39 & -17.7 & $84.04 \pm 0.71$ & $1520 \pm 70$ \\
HAM-2709 & $40-42$ & 6.1 & 0.31 & -17.7 & $81.72 \pm 0.70$ & $1740 \pm 70$ \\
HAM-2710 & $42-44$ & 6.0 & 0.31 & -18.6 & $135.60 \pm 0.88$ & Modern \\
HAM-2711 & $44-46$ & 6.2 & 0.29 & -18.8 & $91.77 \pm 0.73$ & $790 \pm 70$ \\
HAM-2712 & $46-48$ & 6.2 & 0.28 & -18.6 & $77.52 \pm 0.52$ & $2150 \pm 50$ \\
HAM-2713 & $48-50$ & 6.4 & 0.27 & -18.9 & $76.37 \pm 0.69$ & $2260 \pm 70$ \\
HAM-2714 & $50-60$ & 6.1 & 0.25 & -19.2 & $67.99 \pm 0.91$ & $3190 \pm 110$ \\
HAM-2715 & $60-70$ & 6.1 & 0.18 & -19.3 & $62.46 \pm 0.63$ & $3870 \pm 80$ \\
HAM-2716 & $70-80$ & 6.2 & 0.16 & -19.2 & $61.91 \pm 0.63$ & $3940 \pm 80$ \\
HAM-2717 & $80-90$ & 6.2 & 0.16 & -18.8 & $70.35 \pm 0.50$ & $2930 \pm 60$ \\
HAM-2718 & $90-100$ & 6.2 & 0.19 & -19.3 & $49.78 \pm 0.59$ & $5700 \pm 100$ \\
\hline
\end{tabular}

\section{Chum Pae}

\section{Profile-Related Data}

Location (longitude, latitude):

$16^{\circ} 33^{\prime} \mathrm{N}, 102^{\circ} 02^{\prime} \mathrm{E}$

Location (country, next city or village):

Thailand, rice experimental station at Chum Pae near Khon Kaen

Soil order and type (USDA classification): Aeric Paleaquult, loamy, mixed, nonacid, isohyperthermic

Soil order and type (local classification):

Roi Et series

Mean annual temperature:

Annual rainfall:

Vegetation and land use:

$28^{\circ} \mathrm{C}$

$1100 \mathrm{~mm}$

Site description:

Rice

The Chum Pae rice experimental station is located at the depressed part of the Korat plateau in Northeast Thailand in an undulating region with limited rainfall. The groundwater table is below $300 \mathrm{~cm}$ during the peak of the dry season. At the time of sampling, the soil was dry down to $170 \mathrm{~cm}$.

Date of sampling:

Date of measurement:

Collector:

Submitter:

Submitter's comment:

Becker-Heidmann

Becker-Heidmann

The carbon content is very low, not only in the subsoil, but especially in the topsoil and shows no difference between topsoil and subsoil. $\delta^{13} \mathrm{C}$ is rather high but not unusual for tropical rice soils. The increase with depth from -21 to $-18 \%$ is lower than in the other studied soils, indicating a weak decomposition. The fact that the ${ }^{14} \mathrm{C}$ activity is as high as the atmospheric one in the plowed horizon also indicates low decomposition. Decomposition is slow in this puddled soil with low and imbalanced nutrient supply, high bulk density and low biological diversity and activity, as was shown also by decomposition experiments with ${ }^{14} \mathrm{C}$-labeled rice straw, which were conducted in the same field after the samples for this study were taken (Snitwongse 
et al. 1988). During the first year, 55-60\% of the straw was lost. The half-life of the resistant part of the added organic matter was $13.5 \mathrm{yr}$ in the submerged planted soil followed by dry fallow. After $3 \mathrm{yr}, 30 \%$ was found within $50 \mathrm{~cm}$ depth.

Literature references:

Becker-Heidmann (1990); Scharpenseel et al. (1989);

Scharpenseel and Becker-Heidmann (1993);

Scharpenseel and Becker-Heidmann (1994b)

\section{Description of the Profile at Chum Pae Station}

Depth $(\mathrm{cm})$ Horizon Description

0-12 Ap1g $\begin{aligned} & \text { Light yellowish brown (10YR 6/4), silty, aggregate surfaces covered } \\ & \text { with iron }\end{aligned}$

12-20 Ap2g Dark yellowish brown (10YR 4/4), puddling zone

20-38 B1 Dark grayish brown (10YR 4/2), silty clay loam, hard black concretions (manganese) with red covering (iron), soft yellowish red concretions (iron)

38-48 B21tg Dark grayish brown(10YR 4/2), clay loam, probably clay migration, manganese and iron concretions

48-74 B22tg Dark grayish brown, clay loam, probably clay migration, concretions

74-92+ B3g

Dark grayish brown, clay loam, concretions

TABLE 22. Chum Pae

\begin{tabular}{lccccrc}
\hline Lab code & Depth $(\mathrm{cm})$ & $\mathrm{pH}$ & $\mathrm{C}$ & $\delta^{13} \mathrm{C}$ & ${ }^{14} \mathrm{C}$ activity & ${ }^{14} \mathrm{C}$ age \\
\hline HAM-2401 & $0-2$ & 4.2 & 0.93 & -20.4 & $116.72 \pm 0.71$ & Modern \\
HAM-2402 & $2-4$ & 4.4 & 0.20 & -20.7 & $118.20 \pm 0.71$ & Modern \\
HAM-2403 & $4-6$ & 4.5 & 0.33 & -19.8 & $118.07 \pm 0.71$ & Modern \\
HAM-2404 & $6-8$ & 4.5 & 0.48 & -19.5 & $118.67 \pm 0.71$ & Modern \\
HAM-2405 & $8-10$ & 4.8 & 0.48 & -19.6 & $119.37 \pm 0.72$ & Modern \\
HAM-2406 & $10-12$ & 5.0 & 0.29 & -20.1 & $116.12 \pm 0.70$ & Modern \\
HAM-2407 & $12-14$ & 5.7 & 0.43 & -19.4 & $101.65 \pm 0.84$ & Modern \\
HAM-2408 & $14-16$ & 6.2 & 0.33 & -19.0 & $96.78 \pm 0.73$ & $360 \pm 60$ \\
HAM-2409 & $16-18$ & 5.0 & 0.32 & -18.6 & $92.07 \pm 0.61$ & $770 \pm 50$ \\
HAM-2410 & $18-20$ & 6.7 & 0.37 & -18.6 & $102.50 \pm 0.65$ & Modern \\
HAM-2411 & $20-23$ & 6.5 & 0.40 & -18.5 & $95.15 \pm 0.73$ & $510 \pm 60$ \\
HAM-2412 & $23-24$ & 6.0 & 0.42 & -18.5 & $93.85 \pm 0.72$ & $610 \pm 60$ \\
HAM-2413 & $24-26$ & 5.8 & 0.41 & -18.3 & $99.37 \pm 0.64$ & $160 \pm 50$ \\
HAM-2414 & $26-28$ & 5.9 & 0.25 & -18.6 & $98.19 \pm 0.63$ & $250 \pm 50$ \\
HAM-2415 & $28-30$ & 5.8 & 0.28 & -18.2 & $94.94 \pm 0.72$ & $530 \pm 60$ \\
HAM-2416 & $30-32$ & 5.8 & 0.26 & -18.2 & $93.79 \pm 0.72$ & $630 \pm 60$ \\
HAM-2417 & $32-34$ & 5.5 & 0.41 & -18.1 & $94.14 \pm 0.73$ & $600 \pm 60$ \\
HAM-2418 & $34-36$ & 5.4 & 0.45 & -18.3 & $93.32 \pm 0.72$ & $660 \pm 60$ \\
HAM-2419 & $36-38$ & 5.7 & 0.24 & -18.4 & $98.01 \pm 0.64$ & $270 \pm 50$ \\
HAM-2420 & $38-40$ & 5.9 & 0.36 & -18.5 & $92.26 \pm 0.72$ & $750 \pm 60$ \\
HAM-2421 & $40-42$ & 5.6 & 0.23 & -18.4 & $93.30 \pm 0.72$ & $660 \pm 60$ \\
HAM-2422 & $42-44$ & 5.9 & 0.19 & -18.8 & $92.47 \pm 0.71$ & $730 \pm 60$ \\
HAM-2423 & $44-46$ & 5.0 & 0.39 & -18.5 & $95.39 \pm 0.63$ & $480 \pm 50$ \\
HAM-2424 & $46-48$ & 5.3 & 0.25 & -18.6 & $96.90 \pm 0.63$ & $360 \pm 50$ \\
HAM-2425 & $48-50$ & 5.1 & 0.33 & -18.8 & $93.76 \pm 0.62$ & $620 \pm 50$ \\
HAM-2426 & $50-52$ & 6.0 & 0.35 & -19.0 & $95.70 \pm 0.73$ & $450 \pm 60$ \\
HAM-2427 & $52-54$ & 4.9 & 0.33 & -18.8 & $93.88 \pm 0.62$ & $610 \pm 50$ \\
& & & & & &
\end{tabular}


TABLE 22. Chum Pae (Continued)

\begin{tabular}{lccccrr}
\hline Lab code & Depth $(\mathrm{cm})$ & $\mathrm{pH}$ & $\mathrm{C}$ & $\delta^{13} \mathrm{C}$ & ${ }^{14} \mathrm{C}$ activity & \multicolumn{1}{c}{${ }^{14} \mathrm{C}$ age } \\
\hline HAM-2428 & $54-56$ & 5.3 & 0.25 & -19.0 & $87.74 \pm 0.70$ & $1150 \pm 60$ \\
HAM-2429 & $56-58$ & 5.3 & 0.24 & -19.1 & $87.08 \pm 0.69$ & $1210 \pm 60$ \\
HAM-2430 & $58-60$ & 5.1 & 0.30 & -19.4 & $88.19 \pm 0.69$ & $1100 \pm 60$ \\
HAM-2431 & $60-62$ & 5.0 & 0.23 & -19.3 & $89.90 \pm 0.60$ & $950 \pm 50$ \\
HAM-2432 & $62-64$ & 5.4 & 0.12 & -19.8 & $90.40 \pm 0.60$ & $890 \pm 50$ \\
HAM-2433 & $64-66$ & 5.1 & 0.30 & -19.9 & $86.62 \pm 0.69$ & $1240 \pm 60$ \\
HAM-2434 & $66-68$ & 5.0 & 0.22 & -19.9 & $91.60 \pm 0.61$ & $790 \pm 50$ \\
HAM-2435 & $68-70$ & 5.1 & 0.22 & -20.3 & $88.19 \pm 0.75$ & $1090 \pm 70$ \\
HAM-2436 & $70-72$ & 5.3 & 0.25 & -20.1 & $86.10 \pm 0.72$ & $1280 \pm 70$ \\
HAM-2437 & $72-74$ & 5.2 & 0.28 & -20.4 & $89.61 \pm 0.61$ & $960 \pm 60$ \\
HAM-2438 & $74-76$ & 5.8 & 0.19 & -20.6 & $89.28 \pm 0.60$ & $980 \pm 50$ \\
HAM-2439 & $76-78$ & 5.4 & 0.25 & -20.5 & $88.66 \pm 0.60$ & $1040 \pm 50$ \\
HAM-2440 & $78-80$ & 5.8 & 0.25 & -20.8 & $85.36 \pm 0.69$ & $1340 \pm 70$ \\
HAM-2441 & $80-82$ & 5.9 & 0.18 & -20.9 & $85.86 \pm 0.79$ & $1290 \pm 70$ \\
HAM-2442 & $82-84$ & 5.8 & 0.25 & -20.9 & $87.42 \pm 0.60$ & $1150 \pm 60$ \\
HAM-2443 & $84-86$ & 5.5 & 0.16 & -21.2 & $90.01 \pm 0.62$ & $910 \pm 60$ \\
HAM-2444 & $86-88$ & 5.7 & 0.18 & -21.2 & $87.71 \pm 0.60$ & $1110 \pm 60$ \\
HAM-2466 & $88-90$ & 6.2 & 0.23 & -21.4 & $88.77 \pm 0.60$ & $1020 \pm 60$ \\
HAM-2467 & $90-92$ & 5.8 & 0.16 & -21.3 & $83.98 \pm 0.68$ & $1460 \pm 70$ \\
\hline
\end{tabular}

\section{KLONG LUANG}

\section{Profile-Related Data}

Location (longitude, latitude):

Location (country, next city or village):

Soil order and type (USDA classification):

Soil order and type (local classification):

Parent material:

Mean annual temperature:

Annual rainfall:

Vegetation and land use:

Site description:

The soil, located at Klong Luang north of Bangkok, is representative of the acid sulfate soils that cover $c a$. $8000 \mathrm{~km}^{2}$ in the Bangkok Plain. These soils were studied in detail by van Breemen (1976); a similar profile of Rangsit series is described in his book. By ${ }^{14} \mathrm{C}$ dating of peat material, van der Kevie (1972) estimated the formation of the tidal marsh deposits at $c a .3100 \mathrm{yr}$ ago.

Date of sampling:

Date of measurement:

Collector:

Submitter:

Submitter's comment:

1984

1986

S. Pongpan, P. Snitwongse

P. Snitwongse, Becker-Heidmann

The $\mathrm{pH}$ is very low throughout the profile due to the high sulfate content. The carbon content is only $1.3 \%$ in the topsoil, but does not decrease much to the subsoil. The $\delta^{13} \mathrm{C}$ value near the surface is the normal one for tropical rice soils ( $c f$. the other soils in this study), but the increase with depth in the topsoil of this profile is the lowest. The ${ }^{14} \mathrm{C}$ activity in this soil is one of the lowest of all studied rice soils. Together with the small increase of $\delta^{13} \mathrm{C}$, it suggests that there has been very little input of organic matter. Otherwise, the ${ }^{14} \mathrm{C}$ age of the subsoil could not have reached the age of the sediments, mentioned above in the soil description (cf. van der Kevie 1972). 
Literature references:

Becker-Heidmann (1990)

Description of the Profile at Klong Luang:

\begin{tabular}{|c|c|c|}
\hline Depth $(\mathrm{cm})$ & Horizon & Description \\
\hline $0-10$ & Ap1g & $\begin{array}{l}\text { Black (10YR 2/1), yellowish brown (10YR 5/6) mottles, frequent very } \\
\text { fine roots }\end{array}$ \\
\hline $10-22$ & Ap2g & Black, few yellowish brown and red mottles, many very fine roots \\
\hline $22-30$ & A2 $g$ & Brown to dark grayish brown, very few very fine roots \\
\hline $30-42$ & B1g & Brown to dark brown, brown and red mottles, few very fine roots \\
\hline $42-58$ & B21g & $\begin{array}{l}\text { Brown to dark brown, many red and "straw" yellow mottles, very few } \\
\text { very fine roots }\end{array}$ \\
\hline $58+$ & $\mathrm{B} 22 \mathrm{~g}$ & Brown, "straw" yellow mottle, very few very fine roots \\
\hline
\end{tabular}

TABLE 23. Klong Luang

\begin{tabular}{lccccrc}
\hline Lab code & Depth $(\mathrm{cm})$ & $\mathrm{pH}$ & $\mathrm{C}$ & $\delta^{13} \mathrm{C}$ & \multicolumn{1}{c}{${ }^{14} \mathrm{C}$ activity } & ${ }^{14} \mathrm{C}$ age \\
\hline HAM-2468 & $0-2$ & 4.3 & 1.39 & -22.6 & $106.01 \pm 0.67$ & Modern \\
HAM-2469 & $2-4$ & 4.4 & 1.31 & -22.2 & $105.12 \pm 0.66$ & Modern \\
HAM-2470 & $4-6$ & 4.6 & 1.32 & -22.2 & $99.99 \pm 0.74$ & $50 \pm 60$ \\
HAM-2471 & $6-8$ & 4.6 & 1.32 & -21.9 & $104.83 \pm 0.66$ & Modern \\
HAM-2472 & $8-10$ & 4.3 & 1.41 & -22.2 & $98.87 \pm 0.79$ & $140 \pm 60$ \\
HAM-2473 & $10-12$ & 4.4 & 1.38 & -22.0 & $100.27 \pm 0.75$ & Modern \\
HAM-2474 & $12-14$ & 4.5 & 1.31 & -21.2 & $104.03 \pm 0.70$ & Modern \\
HAM-2475 & $14-16$ & 4.5 & 1.21 & -21.7 & $102.39 \pm 0.65$ & Modern \\
HAM-2476 & $16-18$ & 4.3 & 1.25 & -20.8 & $89.67 \pm 0.70$ & $940 \pm 60$ \\
HAM-2477 & $18-20$ & 4.3 & 1.27 & -20.2 & $95.60 \pm 0.63$ & $440 \pm 50$ \\
HAM-2478 & $20-22$ & 4.1 & 1.25 & -20.4 & $85.71 \pm 0.68$ & $1310 \pm 60$ \\
HAM-2479 & $22-24$ & 4.0 & 1.30 & -20.4 & $85.39 \pm 0.59$ & $1340 \pm 60$ \\
HAM-2480 & $24-26$ & 4.0 & 1.21 & -21.0 & $80.22 \pm 0.68$ & $1840 \pm 70$ \\
HAM-2481 & $26-28$ & 4.2 & 1.15 & -21.2 & $83.23 \pm 0.60$ & $1540 \pm 60$ \\
HAM-2482 & $28-30$ & 3.8 & 1.10 & -20.9 & $81.20 \pm 0.67$ & $1740 \pm 70$ \\
HAM-2483 & $30-32$ & 4.3 & 0.98 & -21.3 & $77.94 \pm 0.69$ & $2060 \pm 70$ \\
HAM-2484 & $32-34$ & 4.2 & 0.84 & -21.2 & $80.39 \pm 0.57$ & $1820 \pm 60$ \\
HAM-2485 & $34-36$ & 3.7 & 0.81 & -21.3 & $75.51 \pm 0.65$ & $2320 \pm 70$ \\
HAM-2486 & $36-38$ & 4.2 & 0.86 & -21.1 & $79.21 \pm 0.56$ & $1930 \pm 60$ \\
HAM-2487 & $38-40$ & 3.9 & 0.79 & -21.3 & $73.10 \pm 0.63$ & $2580 \pm 70$ \\
HAM-2488 & $40-42$ & 4.0 & 0.78 & -21.0 & $75.84 \pm 0.55$ & $2290 \pm 60$ \\
HAM-2489 & $42-44$ & 4.0 & 0.72 & -21.0 & $71.58 \pm 0.63$ & $2750 \pm 70$ \\
HAM-2490 & $44-46$ & 4.1 & 0.75 & -21.0 & $74.94 \pm 0.55$ & $2380 \pm 60$ \\
HAM-2491 & $46-48$ & 4.0 & 0.74 & -21.2 & $68.70 \pm 0.61$ & $3080 \pm 70$ \\
HAM-2492 & $48-50$ & 4.2 & 0.80 & -21.1 & $74.04 \pm 0.55$ & $2480 \pm 60$ \\
HAM-2493 & $50-52$ & 4.0 & 0.79 & -21.1 & $69.77 \pm 0.65$ & $2950 \pm 70$ \\
HAM-2494 & $52-54$ & 3.8 & 0.84 & -21.3 & $73.02 \pm 0.54$ & $2590 \pm 60$ \\
HAM-2495 & $54-56$ & 3.9 & 0.77 & -21.4 & $68.82 \pm 0.69$ & $3060 \pm 80$ \\
HAM-2496 & $56-58$ & 4.0 & 0.84 & -21.7 & $67.15 \pm 0.92$ & $3250 \pm 110$ \\
HAM-2497 & $58-60$ & 4.2 & 0.88 & -21.8 & $70.74 \pm 0.54$ & $2830 \pm 60$ \\
HAM-2498 & $60-62$ & 4.4 & 0.85 & -21.9 & $65.47 \pm 0.69$ & $3450 \pm 90$ \\
HAM-2499 & $62-64$ & 4.3 & 0.84 & -22.2 & $66.36 \pm 0.60$ & $3340 \pm 70$ \\
\hline
\end{tabular}




\section{Tachiat, Profile 1}

\section{Profile-Related Data}

Location (longitude, latitude):

$7^{\circ} 22^{\prime} \mathrm{N}, 100^{\circ} 10^{\prime} \mathrm{E}$

Location (country, next city or village):

Thailand, Tachiat, $c a .60 \mathrm{~km}$ northwest of Songkhla

Soil order and type (FAO classification):

Gleyic Acrisol

Soil order and type (USDA classification):

Soil order and type (local classification):

Parent material:

Typic Paleaquult, clayey-kaolonitic isohyperthermic

Bang Nara series

Mean annual temperature:

Marine sediment

Annual rainfall:

$27.6^{\circ} \mathrm{C}$

Vegetation and land use:

\section{$2500 \mathrm{~mm}$}

Farmers have grown rice at this site since land clearing $>40 \mathrm{yr}$ ago. For the last $20 \mathrm{yr}$, rice has been planted nearly every rainy season, while during the dry season the soil was allowed to lie fallow.

Site description: $20 \mathrm{~m}$ asl

The field was located $c a .10 \mathrm{~km}$ from the lagoon of Phattalung on the experimental site of the Soils Department of Prince of Songkhla University. Puddling was usually done to a depth of $\sim 13 \mathrm{~cm}$. The hardpan was at ca. $40-50 \mathrm{~cm}$ depth. The surface had previously been undulating, but was leveled only a short time before sampling. Profile 1 was nearly unaffected by this, whereas Profile 2 was prepared within the former lower and now filled part of the field. The groundwater table at time of sampling was at $56 \mathrm{~cm}$ depth.

Date of sampling:

1990

Date of measurement:

1991

Collector:

Submitter:

Submitter's comment:

Becker-Heidmann, Apinan Kamnalrut

Becker-Heidmann, Apinan Kamnalrut

The $\mathrm{pH}$ of the Tachiat soils is very low. Both soils have a pH of only 4, with nearly no variation throughout the profile. The soil contains no carbonate, and the carbon content is also rather low. The low $\delta^{13} \mathrm{C}$ value indicates little decomposition and humus maintanance. The only slight enrichment of $\delta^{13} \mathrm{C}$ between the surface soil and the bottom of the puddling layer supports this interpretation. In particular, no additional anaerobic decomposition with methane production can be assumed based on this depth distribution. In the subsoil, the $\delta^{13} \mathrm{C}$ value decreases, which means that there is no considerable transport of freshly decomposed organic matter into the deeper part of the soil. The ${ }^{14} \mathrm{C}$ activity in the topsoil reflects the actual ${ }^{14} \mathrm{C}$ activity of the atmosphere, supporting the thesis of reduced decomposition. Below the puddling zone, the ${ }^{14} \mathrm{C}$ activity sharply decreases, again indicating impeded percolation, down to a value of $84 \mathrm{pMC}$, which corresponds to a ${ }^{14} \mathrm{C}$ age of $c a .1400 \mathrm{BP}$. Obviously, the soil development within this alluvial sediment is relatively young. The comparable rice soil at Klong Luang shows ${ }^{14} \mathrm{C}$ ages up to $3400 \mathrm{BP}$.

Literature references:

Becker-Heidmann (1992); Drachenberg (1992)

\section{Description of Profile at Tachiat 1}

\begin{tabular}{|c|c|c|}
\hline Depth / cm & Horizon & Description \\
\hline $0-18$ & Apg & Brown (10 YR 5/3), with reddish mottles \\
\hline $18-30$ & B1g & $\begin{array}{l}\text { Very pale brown (10YR 7/4), spotted, with microcracks coated with iron } \\
\text { (5YR } 5 / 8)\end{array}$ \\
\hline $30-40$ & B21g & Light gray (10YR 7/2), microcracks coated (5YR 5/8) \\
\hline $40-52$ & B22g & Brown with red mottles (10YR $4 / 8$ ), high coarse sand content \\
\hline $52+$ & B23g & Very pale brown with red mottles, water table at $56 \mathrm{~cm}$ \\
\hline
\end{tabular}


TABLE 24. Tachiat, Profile 1

\begin{tabular}{lcccccc}
\hline Lab code & Depth $(\mathrm{cm})$ & $\mathrm{pH}$ & $\mathrm{C}$ & $\delta^{13} \mathrm{C}$ & ${ }^{14} \mathrm{C}$ activity & ${ }^{14} \mathrm{C}$ age \\
\hline HAM-3070 & $0-2$ & 4.1 & 1.07 & -25.9 & $105.07 \pm 0.75$ & Modern \\
HAM-3071 & $2-4$ & 4.0 & 1.05 & -24.6 & $105.76 \pm 0.76$ & Modern \\
HAM-3072 & $4-6$ & 3.8 & 0.97 & -24.7 & $106.06 \pm 0.76$ & Modern \\
HAM-3073 & $6-8$ & 3.9 & 0.94 & -24.6 & $106.52 \pm 0.75$ & Modern \\
HAM-3074 & $8-10$ & 3.9 & 0.99 & -24.9 & $105.40 \pm 0.75$ & Modern \\
HAM-3075 & $10-12$ & 4.0 & 0.98 & -25.3 & $105.89 \pm 0.75$ & Modern \\
HAM-3076 & $12-14$ & 3.9 & 0.99 & -24.5 & $106.96 \pm 0.74$ & Modern \\
HAM-3077 & $14-16$ & 4.0 & 0.98 & -24.9 & $105.87 \pm 0.74$ & Modern \\
HAM-3078 & $16-18$ & 4.0 & 0.99 & -25.1 & $104.13 \pm 0.74$ & Modern \\
HAM-3079 & $18-20$ & 4.0 & 0.92 & -24.4 & $105.30 \pm 0.74$ & Modern \\
HAM-3080 & $20-22$ & 3.9 & 0.91 & -24.1 & $105.70 \pm 0.75$ & Modern \\
HAM-3081 & $22-24$ & 4.0 & 0.74 & -23.9 & $104.07 \pm 0.74$ & Modern \\
HAM-3082 & $24-26$ & 4.1 & 0.51 & -24.0 & $99.62 \pm 0.72$ & $50 \pm 70$ \\
HAM-3083 & $26-28$ & 4.1 & 0.40 & -24.2 & $96.57 \pm 0.71$ & $290 \pm 70$ \\
HAM-3084 & $28-30$ & 4.0 & 0.38 & -24.5 & $92.80 \pm 0.71$ & $610 \pm 70$ \\
HAM-3085 & $30-32$ & 4.0 & 0.34 & -25.0 & $91.91 \pm 0.69$ & $680 \pm 70$ \\
HAM-3086 & $32-34$ & 3.9 & 0.38 & -24.9 & $90.62 \pm 0.69$ & $790 \pm 70$ \\
HAM-3087 & $34-36$ & 4.0 & 0.33 & -25.2 & $88.72 \pm 0.70$ & $960 \pm 70$ \\
HAM-3088 & $36-38$ & 4.0 & 0.33 & -25.1 & $90.56 \pm 0.70$ & $790 \pm 70$ \\
HAM-3089 & $38-40$ & 3.9 & 0.32 & -25.1 & $88.23 \pm 0.68$ & $1000 \pm 70$ \\
HAM-3090 & $40-42$ & 3.9 & 0.32 & -25.5 & $89.61 \pm 0.69$ & $870 \pm 70$ \\
HAM-3091 & $42-44$ & 3.9 & 0.33 & -25.4 & $88.65 \pm 0.69$ & $960 \pm 70$ \\
HAM-3092 & $44-46$ & 3.9 & 0.32 & -25.3 & $86.63 \pm 0.68$ & $1150 \pm 70$ \\
HAM-3093 & $46-48$ & 3.9 & 0.32 & -25.6 & $86.40 \pm 0.70$ & $1160 \pm 70$ \\
HAM-3094 & $48-50$ & 4.0 & 0.31 & -25.3 & $86.09 \pm 0.69$ & $1200 \pm 70$ \\
HAM-3095 & $50-52$ & 3.9 & 0.31 & -25.4 & $86.00 \pm 0.67$ & $1200 \pm 70$ \\
HAM-3096 & $52-54$ & 3.9 & 0.30 & -25.6 & $85.52 \pm 0.68$ & $1250 \pm 70$ \\
HAM-3097 & $54-56$ & 4.0 & 0.31 & -25.6 & $83.50 \pm 0.67$ & $1440 \pm 70$ \\
HAM-3098 & $56-58$ & 4.0 & 0.31 & -25.6 & $84.08 \pm 0.67$ & $1380 \pm 70$ \\
HAM-3099 & $58-60$ & 4.0 & 0.31 & -25.9 & $85.73 \pm 0.68$ & $1220 \pm 70$ \\
HAM-3100 & $60-62$ & 4.0 & 0.30 & -25.4 & $85.91 \pm 0.71$ & $1210 \pm 70$ \\
\hline
\end{tabular}

\section{Tachiat, Profile 2}

\section{Profile-Related Data}

Same as Profile 1 except:

Date of measurement:

1991/1992

Submitter's comment:

The second profile does not differ from the first one regarding $\mathrm{pH}$ and carbon content in general. Only the humus layer is higher, as explained above. The $\delta^{13} \mathrm{C}$ value is nearly the same within the topsoil. There is one exceptionally high value at $c a .17 \mathrm{~cm}$ depth, but the $\delta^{13} \mathrm{C}$ value decreases in the subsoil and is generally lower than in Profile 1 . Thus, we cannot detect a loss of organic substances by percolation. ${ }^{14} \mathrm{C}$ activity is slightly higher than in Profile 1, which means that the decomposition is even less intensive. The corresponding ${ }^{14} \mathrm{C}$ age within the subsoil reaches only $c a .1100 \mathrm{BP}$.

Literature references:

Becker-Heidmann 1992; Drachenberg 1992

\section{Description of Profile at Tachiat 2}

Depth / cm Horizon Description

0-14 Ap1g Dark gray with mottles, cracks, probably deposited Ap material




$\begin{array}{lll}14-32 & \text { Ap2g } & \text { Dark gray with more mottles (Fe?), probably original Ap } \\ 32-52 & \text { B1g } & \text { Light gray with orange-brown mottles (5YR 5/8) } \\ 52+ & \text { B2g } & \begin{array}{l}\text { Orange-brown-light gray mottled, red mottles (less than at Tachiat 1), } \\ \text { water table at } 56 \mathrm{~cm}\end{array}\end{array}$

TABLE 25. Tachiat, Profile 2

\begin{tabular}{lcccccc}
\hline Lab code & Depth $(\mathrm{cm})$ & $\mathrm{pH}$ & $\mathrm{C}$ & $\delta^{13} \mathrm{C}$ & ${ }^{14} \mathrm{C}$ activity & ${ }^{14} \mathrm{C}$ age \\
\hline HAM-3101 & $0-2$ & 4.1 & 1.13 & -25.0 & $105.65 \pm 0.73$ & Modern \\
HAM-3102 & $2-4$ & 4.1 & 1.15 & -24.9 & $105.54 \pm 1.07$ & Modern \\
HAM-3103 & $4-6$ & 4.1 & 1.10 & -25.1 & $105.40 \pm 0.78$ & Modern \\
HAM-3104 & $6-8$ & 4.0 & 1.13 & -24.9 & $105.02 \pm 0.76$ & Modern \\
HAM-3105 & $8-10$ & 4.1 & 1.20 & -24.8 & $105.94 \pm 0.74$ & Modern \\
HAM-3106 & $10-12$ & 4.1 & 1.12 & -24.8 & $109.99 \pm 0.76$ & Modern \\
HAM-3107 & $12-14$ & 4.1 & 1.12 & -25.3 & $107.53 \pm 0.75$ & Modern \\
HAM-3108 & $14-16$ & 4.1 & 1.01 & -25.0 & $106.62 \pm 0.74$ & Modern \\
HAM-3109 & $16-18$ & 4.1 & 0.96 & -24.9 & $109.81 \pm 0.75$ & Modern \\
HAM-3110 & $18-20$ & 4.1 & 1.00 & -25.3 & $110.34 \pm 0.75$ & Modern \\
HAM-3111 & $20-22$ & 4.1 & 0.85 & -25.2 & $110.01 \pm 0.82$ & Modern \\
HAM-3112 & $22-24$ & 4.0 & 0.96 & -25.3 & $105.71 \pm 0.76$ & Modern \\
HAM-3113 & $24-26$ & 4.2 & 0.86 & -25.6 & $109.06 \pm 0.74$ & Modern \\
HAM-3114 & $26-28$ & 4.2 & 0.64 & -26.0 & $104.14 \pm 0.74$ & Modern \\
HAM-3115 & $28-30$ & 4.2 & 0.45 & -26.1 & $101.31 \pm 0.73$ & Modern \\
HAM-3116 & $30-32$ & 4.2 & 0.38 & -26.2 & $96.49 \pm 0.72$ & $270 \pm 70$ \\
HAM-3117 & $32-34$ & 4.2 & 0.30 & -26.9 & $94.58 \pm 0.71$ & $420 \pm 70$ \\
HAM-3118 & $34-36$ & 4.0 & 0.29 & -26.5 & $93.88 \pm 0.72$ & $480 \pm 70$ \\
HAM-3119 & $36-38$ & 4.0 & 0.30 & -26.4 & $92.36 \pm 0.69$ & $620 \pm 70$ \\
HAM-3120 & $38-40$ & 4.1 & 0.30 & -26.5 & $91.90 \pm 0.69$ & $650 \pm 70$ \\
HAM-3121 & $40-42$ & 4.0 & 0.31 & -27.0 & $93.27 \pm 0.71$ & $530 \pm 70$ \\
HAM-3122 & $42-44$ & 4.1 & 0.29 & -26.8 & $89.20 \pm 0.69$ & $890 \pm 70$ \\
HAM-3123 & $44-46$ & 4.1 & 0.31 & -26.6 & $92.77 \pm 0.71$ & $580 \pm 70$ \\
HAM-3124 & $46-48$ & 4.1 & 0.31 & -26.7 & $92.69 \pm 0.71$ & $580 \pm 70$ \\
HAM-3125 & $48-50$ & 4.0 & 0.31 & -26.1 & $90.16 \pm 0.72$ & $810 \pm 70$ \\
HAM-3126 & $50-52$ & 4.0 & 0.29 & -26.9 & $89.36 \pm 0.72$ & $870 \pm 70$ \\
HAM-3127 & $52-54$ & 4.1 & 0.29 & -27.3 & $87.82 \pm 0.69$ & $1010 \pm 70$ \\
HAM-3128 & $54-56$ & 4.1 & 0.28 & -27.6 & $87.19 \pm 0.68$ & $1060 \pm 70$ \\
HAM-3129 & $56-58$ & 4.1 & 0.28 & -27.7 & $87.94 \pm 0.70$ & $990 \pm 70$ \\
\hline & & & & & &
\end{tabular}

\section{TONSANG}

\section{Profile-Related Data}

Location (longitude, latitude):

$18^{\circ} 52^{\prime} \mathrm{N}, 99^{\circ} 02^{\prime} \mathrm{E}$

Location (country, next city or village):

Thailand, Tonsang near San Sai, $c a .10 \mathrm{~km}$ northeast of Chiang Mai

Soil order and type (USDA classification): Typic Tropaqualf

Soil order and type (local classification):

Mean annual temperature:

Low humic gley soil, Hang Dong series

$25^{\circ} \mathrm{C}$

$1220 \mathrm{~mm}$

Annual rainfall:

Vegetation and land use:

The experimental site has been cultivated for rice and soybean rotation for $c a .10 \mathrm{yr}$.

Site description:

The soil was very homogeneous. The groundwater level was at $c a .52 \mathrm{~cm}$ depth the first sampling day and rose to $c a .40 \mathrm{~cm}$ the second day. 


$\begin{array}{ll}\text { Date of sampling: } & 1991 \\ \text { Date of measurement: } & 1992 \\ \text { Collector: } & \text { Becker-Heidmann, Drachenberg, Arayangkoon } \\ \text { Submitter: } & \text { Becker-Heidmann, Arayangkoon }\end{array}$

Submitter's comment:

The $\mathrm{pH}$ value is generally high, $c a .6 .3$ throughout the $\mathrm{B} 1$ and $\mathrm{B} 2 \mathrm{~g}$ horizons and the surface layer. Amazingly, the $\mathrm{pH}$ depth distribution curve shows a minimum of 5.6 between 6 and $10 \mathrm{~cm}$ depth, which is the main rooting zone. The carbon content is very low because of either rapid turnover or low input of organic crop residues. The decrease of carbon with depth within the topsoil, which is unusual for a plowed horizon, supports the rapid turnover hypothesis. The $\delta^{13} \mathrm{C}$ value is generally high at the same level as the other studied tropical soils. If no $\mathrm{C}_{4}$ plant has been grown in the past, the high value of $22 \%$ may be, in principle, a result of either anaerobic decomposition with methane production, or decomposing $\mathrm{C}_{4}$ weeds, or exudation of carbohydrates from the roots during the past when the plot was used for rice. The increase from top to bottom of the Ap horizon by $\mathrm{ca}$. 3\%o stands for high microbiological activity. The decrease within the B2g horizon is due to its anaerobic conditions (Becker-Heidmann 1990; Stout, Goh and Rafter 1981). The varying value near the top of the $\mathrm{B} 2 \mathrm{~g}$ horizon is probably a result of changes in the groundwater table. The depth distributions of carbon content, $\delta^{13} \mathrm{C}$ value and ${ }^{14} \mathrm{C}$ activity indicate a high decomposition rate rather than low input of organic matter.

Literature references:

Becker-Heidmann (1992)

\section{Description of Profile at Tonsang}

\begin{tabular}{|c|c|c|}
\hline Depth / cm & Horizon & Description \\
\hline $0-16$ & Apg & $\begin{array}{l}\text { Loamy clay, dark grayish brown }(2.5 Y 4 / 2) \text {, many fine roots, iron at root } \\
\text { channels }\end{array}$ \\
\hline $16-25$ & B1g & Silty clay, yellowish brown (2.5Y 5/4), many fine roots, red mottles $(\mathrm{Fe})$ \\
\hline $25-52+$ & B2 $g$ & Sandy clayey loam, pale red ( 2.5 YR $6 / 2)$, few roots \\
\hline
\end{tabular}

TABLE 26. Tonsang

\begin{tabular}{lcccccc}
\hline Lab code & Depth $(\mathrm{cm})$ & $\mathrm{pH}$ & $\mathrm{C}$ & ${ }^{13} \mathrm{C}$ & ${ }^{14} \mathrm{C}$ activity & ${ }^{14} \mathrm{C}$ age \\
\hline HAM-2837 & $0-2$ & 6.8 & 1.35 & -22.0 & $117.92 \pm 0.84$ & Modern \\
HAM-2838 & $2-4$ & 6.3 & 1.29 & -21.6 & $119.37 \pm 0.66$ & Modern \\
HAM-2839 & $4-6$ & 5.8 & 1.17 & -21.1 & $120.17 \pm 0.84$ & Modern \\
HAM-2840 & $6-8$ & 5.6 & 1.00 & -21.1 & $120.63 \pm 0.86$ & Modern \\
HAM-2841 & $8-10$ & 5.6 & 0.92 & -21.0 & $122.13 \pm 0.85$ & Modern \\
HAM-2842 & $10-12$ & 5.7 & 0.77 & -21.4 & $121.46 \pm 0.86$ & Modern \\
HAM-2843 & $12-14$ & 6.1 & 0.44 & -20.6 & $115.39 \pm 0.65$ & Modern \\
HAM-2844 & $14-16$ & 6.3 & 0.26 & -19.3 & $105.37 \pm 0.79$ & Modern \\
HAM-2845 & $16-18$ & 6.4 & 0.17 & -19.5 & $101.76 \pm 0.76$ & Modern \\
HAM-2846 & $18-20$ & 6.3 & 0.22 & -19.4 & $101.77 \pm 0.63$ & Modern \\
HAM-2847 & $20-22$ & 6.3 & 0.17 & -20.1 & $100.87 \pm 0.60$ & Modern \\
HAM-2848 & $22-24$ & 6.3 & 0.21 & -19.9 & $100.82 \pm 0.74$ & Modern \\
HAM-2849 & $24-26$ & 6.3 & 0.17 & -20.5 & $101.77 \pm 0.60$ & Modern \\
HAM-2850 & $26-28$ & 6.3 & 0.16 & -20.3 & $100.41 \pm 0.60$ & Modern \\
HAM-2851 & $28-30$ & 6.3 & 0.16 & -20.1 & $96.98 \pm 0.77$ & $330 \pm 70$ \\
HAM-2910 & $30-32$ & 6.3 & 0.16 & -21.0 & $98.38 \pm 0.73$ & $200 \pm 60$ \\
HAM-2911 & $32-34$ & 6.3 & 0.13 & -20.0 & $96.80 \pm 0.62$ & $340 \pm 50$ \\
HAM-2912 & $34-36$ & 6.2 & 0.12 & -21.1 & $95.78 \pm 0.59$ & $410 \pm 50$ \\
HAM-2913 & $36-38$ & 6.3 & 0.11 & -20.6 & $94.87 \pm 0.75$ & $490 \pm 60$ \\
HAM-2914 & $38-40$ & 6.3 & 0.10 & -20.3 & $95.04 \pm 0.73$ & $480 \pm 60$ \\
HAM-2915 & $40-42$ & 6.3 & 0.10 & -20.6 & $95.80 \pm 0.61$ & $420 \pm 50$ \\
\hline
\end{tabular}

